\title{
Asymmetric breaking size-segregation waves in dense granular free-surface flows
}

\author{
P. Gajjar ${ }^{1 \dagger} \dagger$, K. van der $\operatorname{Vaart}^{2}$, A. R. Thornton ${ }^{3}$, C. G. Johnson ${ }^{1}$, C. Ancey ${ }^{2}$ \\ and J. M. N. T. Gray ${ }^{1}$ \\ ${ }^{1}$ School of Mathematics and Manchester Centre for Nonlinear Dynamics, \\ University of Manchester, Manchester M13 9PL, UK \\ ${ }^{2}$ Environmental Hydraulics Laboratory, École Polytechnique Fédérale de Lausanne, \\ Écublens, 1015 Lausanne, Switzerland \\ ${ }^{3}$ Multi-Scale Mechanics Group, MESA+, University of Twente, The Netherlands
}

(Received 23 July 2015; revised 23 December 2015; accepted 27 February 2016; first published online 4 April 2016)

Debris and pyroclastic flows often have bouldery flow fronts, which act as a natural dam resisting further advance. Counter intuitively, these resistive fronts can lead to enhanced run-out, because they can be shouldered aside to form static levees that self-channelise the flow. At the heart of this behaviour is the inherent process of size segregation, with different sized particles readily separating into distinct vertical layers through a combination of kinetic sieving and squeeze expulsion. The result is an upward coarsening of the size distribution with the largest grains collecting at the top of the flow, where the flow velocity is greatest, allowing them to be preferentially transported to the front. Here, the large grains may be overrun, resegregated towards the surface and recirculated before being shouldered aside into lateral levees. A key element of this recirculation mechanism is the formation of a breaking size-segregation wave, which allows large particles that have been overrun to rise up into the faster moving parts of the flow as small particles are sheared over the top. Observations from experiments and discrete particle simulations in a moving-bed flume indicate that, whilst most large particles recirculate quickly at the front, a few recirculate very slowly through regions of many small particles at the rear. This behaviour is modelled in this paper using asymmetric segregation flux functions. Exact non-diffuse solutions are derived for the steady wave structure using the method of characteristics with a cubic segregation flux. Three different structures emerge, dependent on the degree of asymmetry and the non-convexity of the segregation flux function. In particular, a novel 'lens-tail' solution is found for segregation fluxes that have a large amount of non-convexity, with an additional expansion fan and compression wave forming a 'tail' upstream of the 'lens' region. Analysis of exact solutions for the particle motion shows that the large particle motion through the 'lens-tail' is fundamentally different to the classical 'lens' solutions. A few large particles starting near the bottom of the breaking wave pass through the 'tail', where they travel in a region of many small particles with a very small vertical velocity, and take significantly longer to recirculate.

$†$ Email address for correspondence: parmesh.gajjar@alumni.manchester.ac.uk 
Key words: granular media, mixing, pattern formation

\section{Introduction}

Debris and pyroclastic flow deposits often show evidence of bouldery fronts that have a high proportion of large particles (e.g. Sharp \& Nobles 1953; Johnson 1970, 1984; Takahashi 1980; Costa \& Williams 1984; Pierson 1986; Iverson 2014; Turnbull, Bowman \& McElwaine 2015). Figure 1 shows large boulders deposited at the front of a debris flow in Arizona, USA. These large grains tend to be more resistive to downslope motion than the fines, and consequentially have a significant influence on the overall flow dynamics by acting as a 'dam' that resists the flow behind (Pierson 1986). The advancing, more mobile, fine grains from within the interior of the flow (Major \& Iverson 1999) shoulder the large particles at the front to the sides (Johnson et al. 2012), forming coarse-grained levees that channelise the flow. The inside of this channel is lined by a layer of deposited fine grains, further reducing the friction and increasing the run-out distance (Kokelaar et al. 2014). All of this behaviour is readily reproduced in both large- and small-scale experiments (Iverson \& Vallance 2001; Iverson et al. 2010; Johnson et al. 2012). In particular, Pouliquen, Delour \& Savage (1997) observed that the interaction of the resistive front with the mobile interior also causes a lateral instability where the flow-front fingers and breaks into a number of different confining channels (Sharp \& Nobles 1953; Pouliquen et al. 1997; Woodhouse et al. 2012). The development of the bouldery fronts is thus key to understanding how segregation feeds back on the bulk flow field.

A key component within the formation of coarse-grained fronts and lateral levees is the inherent process of size segregation that is common to all polydisperse granular media. Whilst flowing, granular mixtures dilate sufficiently to allow the flow to act like a sieve that naturally sorts the different sized constituents. Small gaps in the grain matrix allow the finer grains to preferentially percolate downwards under gravity, whilst there is a return flow of coarse grains towards the surface. The exact mechanism for the rising of large grains is under investigation (van der Vaart et al. 2015), although the net result is an upward coarsening in the particle-size distribution that is often called inverse grading. For example, a bidisperse mixture containing just two grain sizes would separate into two separate layers in the absence of diffusion, with the large particles on top of the small ones, as shown in figure 2(a). The surface layers have the highest velocities, and so the larger particles are transported to the front of the flow. These coarse grains may then be pushed en masse at the front if massive enough (Pouliquen \& Vallance 1999), or otherwise may be overrun by the advancing flow. They are able to rise up back towards the surface as they are resegregated, creating a complex recirculating motion that connects the upstream inversely graded body of the flow to the coarse-rich flow front. As more large grains are supplied towards the front, the coarse-grained margin grows in size, with the interface propagating forward at a slower speed than the advancing front (Gray \& Kokelaar 2010a,b). The front may obtain a steady size in two dimensions if there is no further upstream supply of large particles, or alternatively, if the upstream supply of large particles is matched by the rate of deposition on the lower basal surface (Gray \& Ancey 2009). The front may also obtain a finite-size steady state in three 


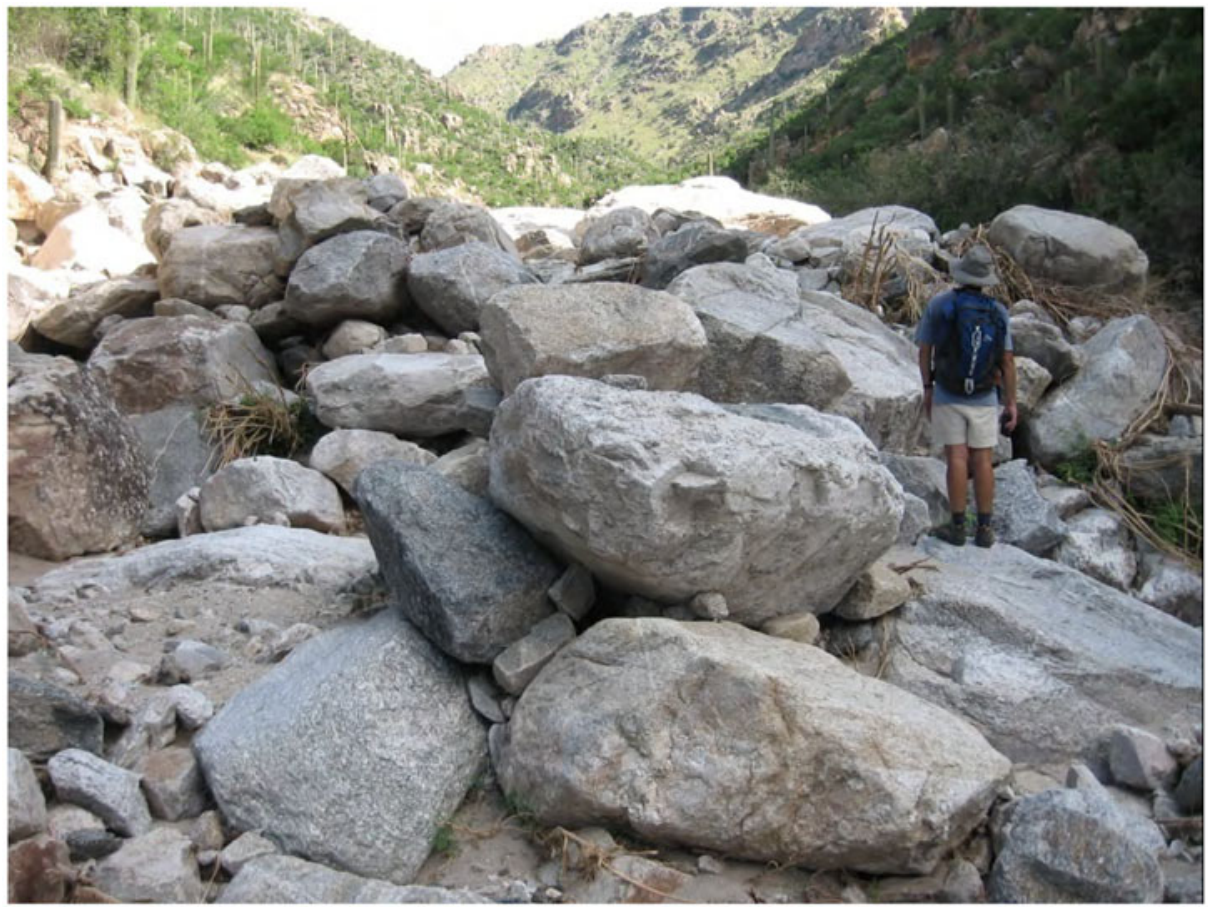

FIgURE 1. Photograph of the front of a debris flow that has stopped in the channel of Rattlesnake Creek, Arizona, USA. The large boulders seen here in the front are typical of many debris and pyroclastic flows, with larger particles segregating upwards to the faster moving surface layers and preferentially transported towards the front, where they accumulate. Photo courtesy of C. Magirl and USGS.

dimensions by shouldering the large grains, transported to the front, laterally outwards to the sides to produce static coarse-grained levees (Johnson et al. 2012; Kokelaar et al. 2014).

\subsection{Recirculating particle motion}

The first real insights into the structure of the recirculation zone were provided by Pouliquen et al. (1997) and Pouliquen \& Vallance (1999), who used a moving camera to approximately measure the lateral recirculating motion of a line of large black crushed fruit stones placed on the surface of a flow of translucent glass beads. Their observations, however, lacked spatial resolution, and further direct experimental observation of the recirculation has been challenging due to its complex time dependence. The recirculation zone propagates quickly downstream at speed $u_{\text {wave }}$ as the front advances forward at speed $u_{\text {front }}$, meaning that there is the difficulty of capturing the motion using a camera moving with the recirculation zone. Long chutes are also required before a steady recirculation regime emerges.

An alternative approach is to use the moving-bed flume set-up shown in figure 3, that is similar to that used by Davies (1990). The flume is $104 \mathrm{~cm}$ in length with a rough $10 \mathrm{~cm}$ wide upward moving conveyor belt positioned between the four stationary vertical walls. The inclination of the channel was set at $19.8^{\circ}$ to establish a uniform flow height along the channel. Higher or lower angles were found to 
(a)

(b)

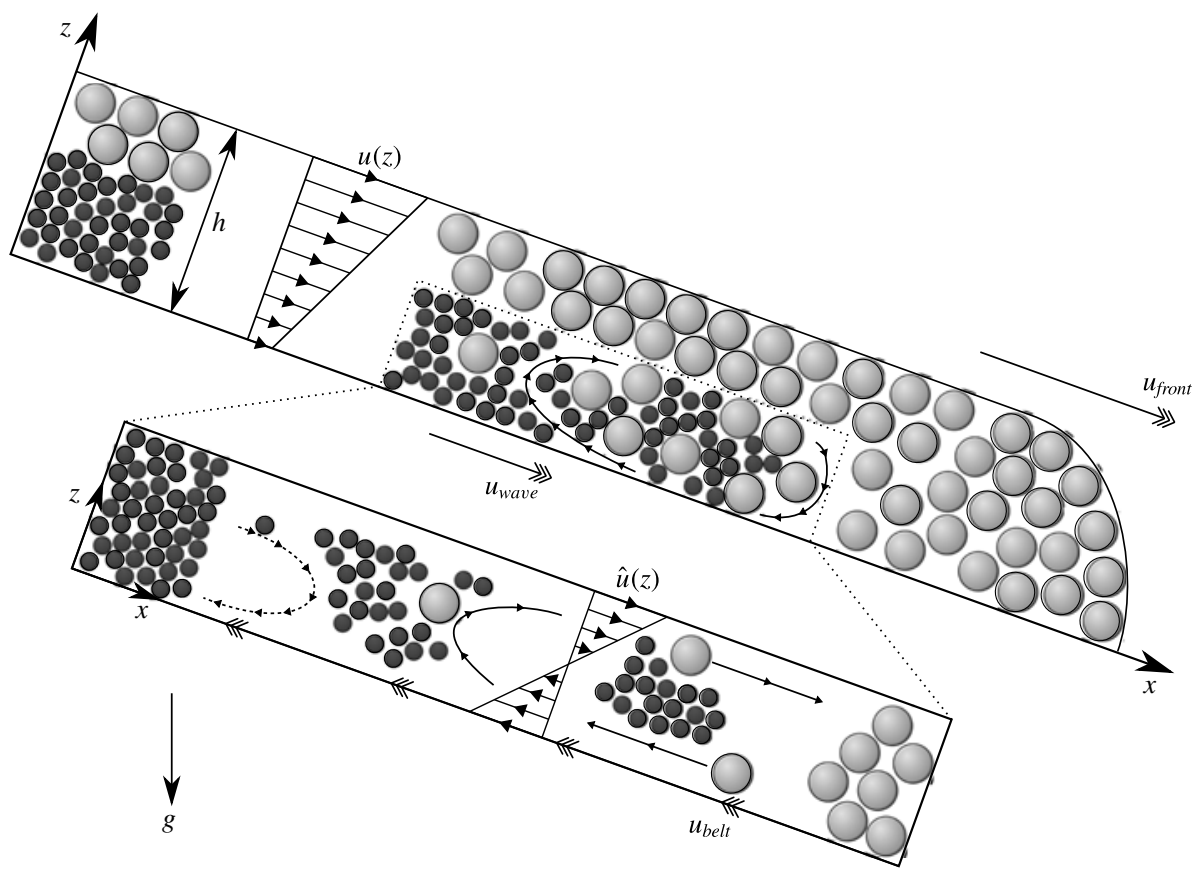

FIGURE 2. (a) A vertical section through a steadily propagating avalanche travelling down an inclined plane. In the body of the flow, the large grains segregate to the upper layers, where the velocity $u(z)$ is greatest, and hence are transported towards the front of the avalanche, where they are overrun, resegregated upwards and recirculated to form a coarse-rich particle front. A complex recirculating motion is created that links the vertically segregated flow in the rear of the avalanche from the coarse-grained front, with the recirculating region known as a 'breaking size-segregation wave' (Thornton \& Gray 2008). Although the front increases in size as more large particles are supplied from the inversely graded flow upstream, the recirculation region shown with dotted lines reaches a steady structure that travels at the average speed $u_{\text {wave }}$ (b) A convenient way of studying this steady recirculation regime is to use a moving-bed flume, which can establish a steady motion within a short chute length. The belt moves upstream at a speed $u_{\text {belt }}$, driving an upstream flow in the lowest layers, whilst the upper layers move downstream under gravity. This generates a net velocity profile $\hat{u}(z)=u(z)-u_{\text {wave }}$ and is the same as examining the recirculation zone within $(a)$ from a frame advecting at speed $u_{\text {wave }}$. There is no upstream supply of large particles in this configuration $(b)$, and so, provided that the segregation and diffusion rates are constant (Thornton \& Gray 2008), it is mathematically equivalent to the subset of figure (a) marked by the dotted lines. Large particles rise towards the surface, and are sheared towards the downstream end of the flume. Some large grains are driven back upstream by the belt, segregate back towards the surface and are recirculated.

cause an accumulation towards the front or rear of the channel, respectively. The belt moves upstream at a velocity $u_{\text {belt }}=72 \mathrm{~mm} \mathrm{~s}^{-1}$. This generates the experimental configuration shown schematically in figure $2(b)$, where the lower layers of the flow are forced upstream by the belt, while the upper layers move downstream under gravity. While this flow is not itself inversely graded, it is mathematically equivalent to the section of an inversely graded avalanche shown in figure 2(a), provided that the segregation and diffusion rates are constant (Thornton \& Gray 2008). The absence 


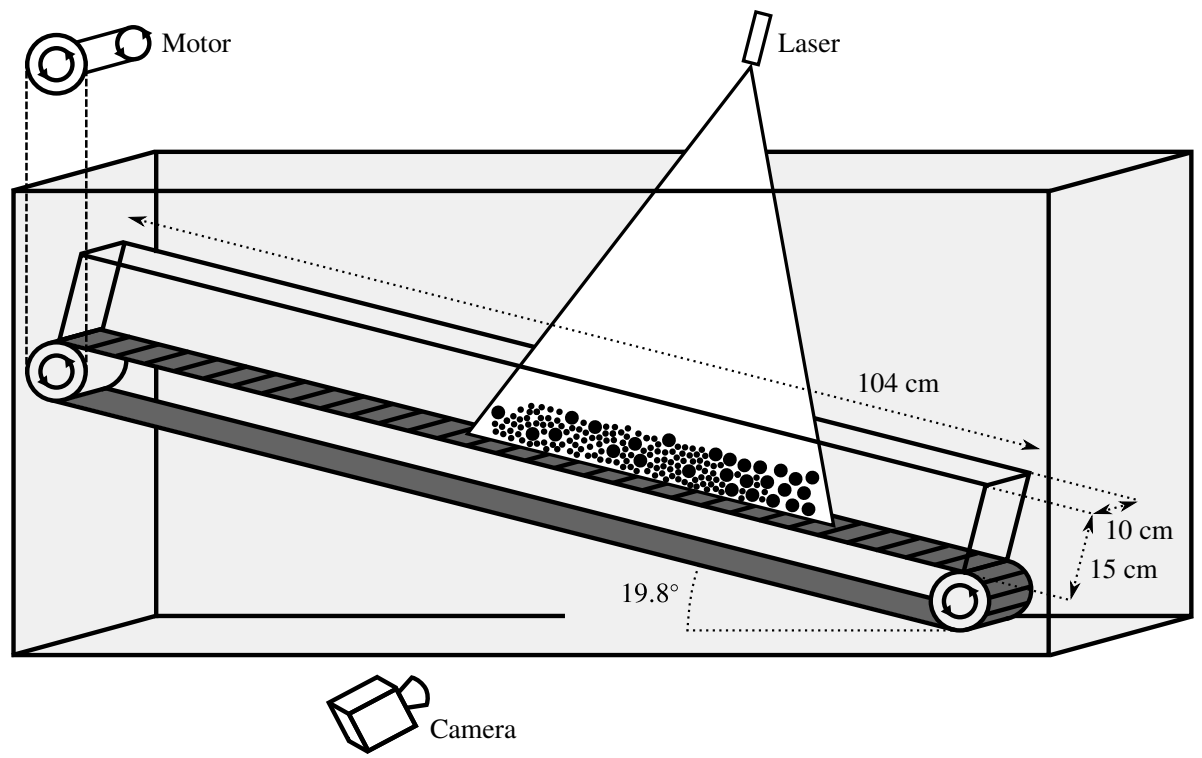

FIgURE 3. A schematic diagram of the moving-bed flume set-up. The flume is $104 \mathrm{~cm}$ in length and $15 \mathrm{~cm}$ high, with a rough $10 \mathrm{~cm}$ wide conveyor belt at the base that moves upstream at velocity $u_{\text {belt }}=72 \mathrm{~mm} \mathrm{~s}^{-1}$. This generates the flow configuration sketched in figure $2(b)$, with the particles in the lower layers of the flow forced upstream by the belt, whilst those in the upper layers of the flow move downstream under gravity. The entire set-up is submerged in a larger tank containing a mixture of benzyl-alcohol and ethanol. This acted as the index matched interstitial fluid, and had a viscosity $\mu=3 \mathrm{mPa} \mathrm{s}$ and fluid density of $995 \mathrm{~kg} \mathrm{~m}^{-3}$. The motor unit was mounted outside of the tank and drove the belt through a chain mechanism. A dye (rhodamine) was added to the fluid and the flow illuminated with a laser sheet of wavelength $532 \mathrm{~nm}$. A camera positioned at one of the glass side walls captured the temporal evolution, with particles appearing as dark circles. The diameters of these circles could be tracked in time to determine whether the particle was small or large. An example snapshot at one moment in time, and the time-averaged concentration fields are shown in figure 6.

of the layer of large particles also allows a steady state to develop within the experimental configuration. Both the experimental configuration and the full problem are assumed to be two-dimensional, meaning that there are no side-wall effects. Just as in the full problem, the large grains in the experimental configuration (figure $2 b$ ) initially segregate upwards and are sheared towards the downstream end of the flume, as shown in the normal exposure photograph in figure 4(a). However, the motion of the belt forces some large grains to be carried upstream, where they subsequently lie below small grains. The large grains resegregate upwards, and once they reach the surface, they are carried back towards the downstream end of the flume. The oblique view in figure 5 looking upstream from the end of the flume clearly shows the accumulated large particles, and resembles the bouldery front shown in figure 1. This moving-bed flume allows the structure of the steady recirculation regime to be examined in greater detail. For example, the long time exposure photograph in $4(b)$, taken with an exposure time of 133 s, illustrates the time-averaged concentration field of the recirculation zone. 
(a)

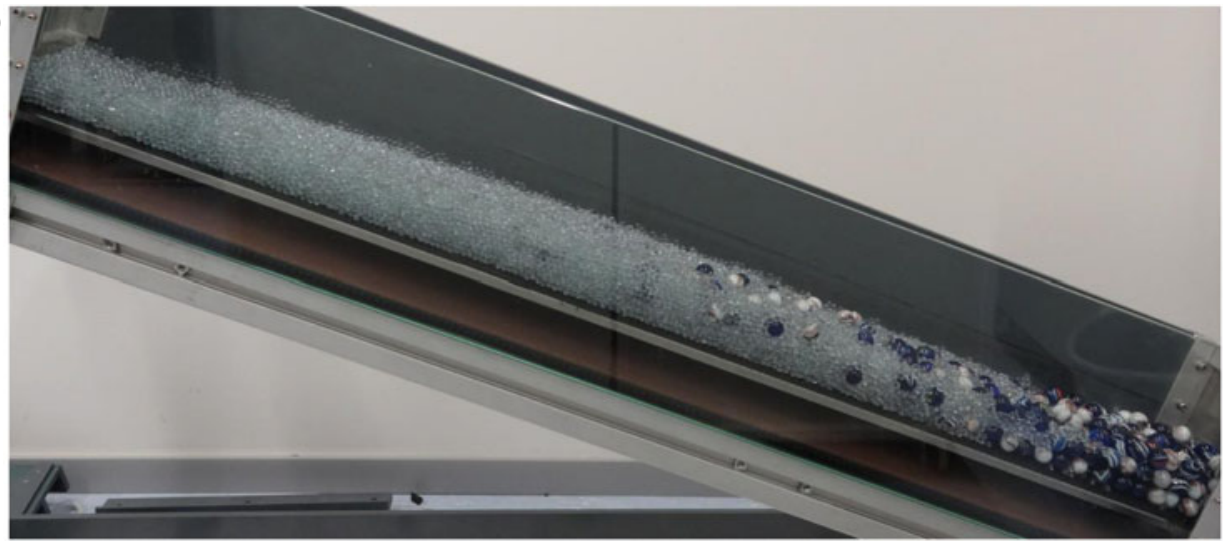

(b)

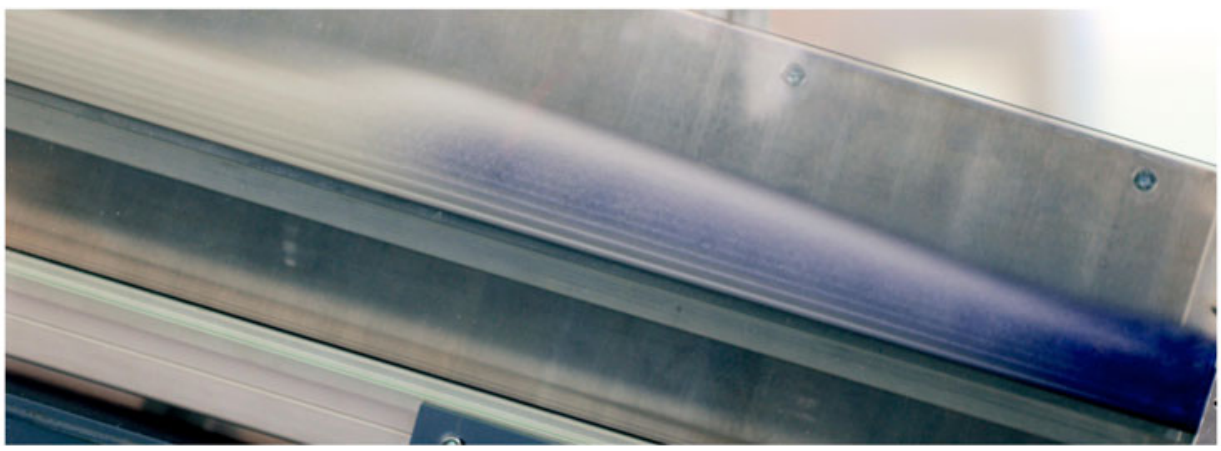

FIGURE 4. Photographs showing the steady recirculation regime established within the $104 \mathrm{~cm}$ long moving-bed flume set-up sketched in figure 3. The particle diameters were 5 and $14 \mathrm{~mm}$. The normal exposure photograph $(a)$ shows the large blue and white marbles collecting towards the right, forming a coarse-rich flow region at the downstream end of the flume, whilst the long exposure photograph $(b)$ shows a time-averaged concentration field and the structure of the breaking size-segregation wave. An exposure time of 133 s was used to capture $(b)$.

The individual motion of the particles on the centre line was revealed using refractive index matched scanning ('RIMS': Wiederseiner et al. 2011a; Dijksman et al. 2012; van der Vaart et al. 2015). Spherical borosilicate glass beads of density $2230 \mathrm{~kg} \mathrm{~m}^{-3}$ and diameters 14 and $5 \mathrm{~mm}$ were used, with the volume ratio of large particles to small particles being 2:5. As shown in figure 3, the entire flume set-up was submerged in a tank containing a mixture of benzyl-alcohol and ethanol, which acted as the index matched interstitial fluid of viscosity $\mu=3 \mathrm{mPa}$, with a fluid density of $995 \mathrm{~kg} \mathrm{~m}^{-3}$. The motor unit for the belt was positioned outside of the tank and drove the belt through a system of chains. A fluorescent dye (rhodamine) was added to the liquid, which was excited by a laser sheet of wavelength $532 \mathrm{~nm}$ in a thin plane parallel to the flow direction. As the particles contain no dye, they appear as dark circles on a bright background. The result is a cross-sectional image of the interior of the flow, which is captured through the glass side wall using a high-speed camera. The laser and camera were positioned to capture the section of the flow containing the recirculation zone. The dark circles are tracked over time, with the minimum and maximum diameters used to determine whether that circle 


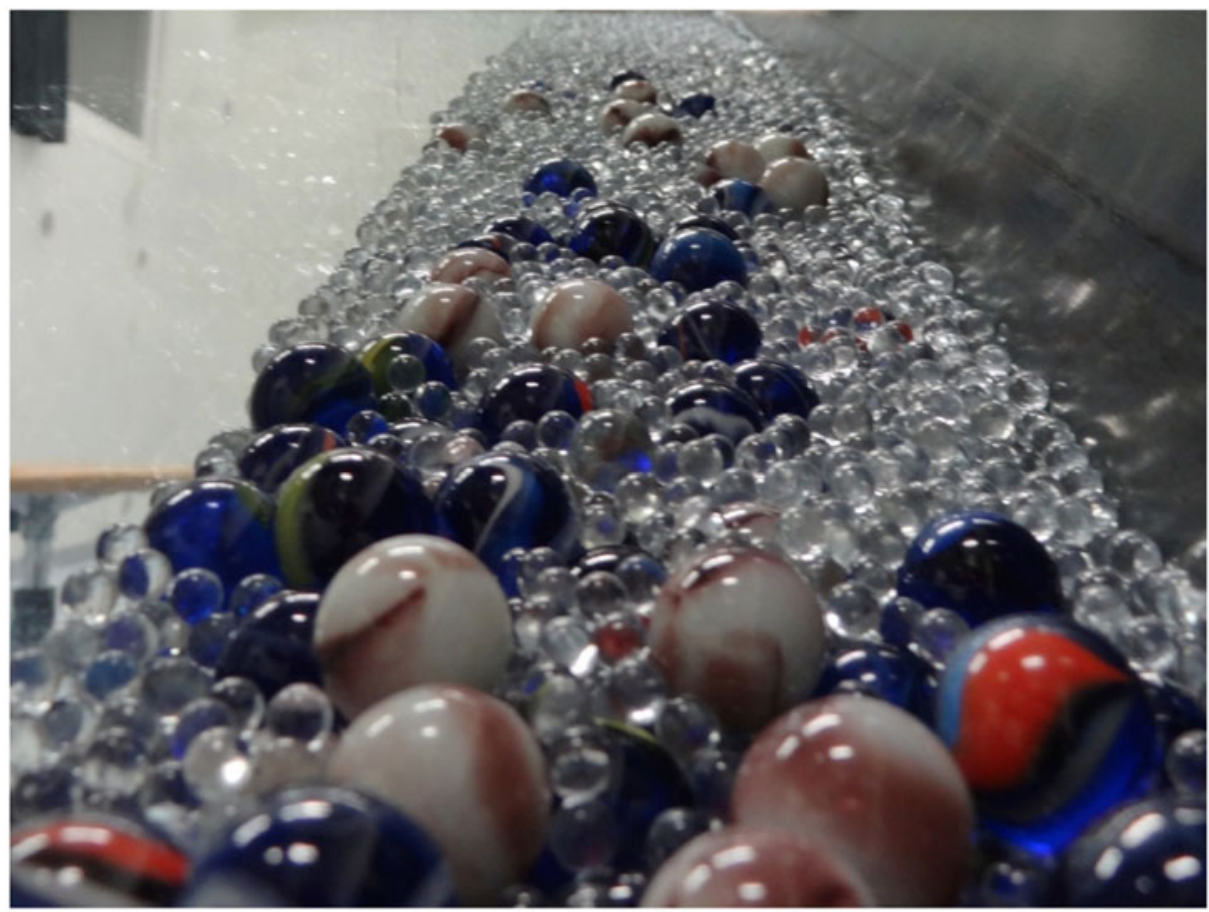

FIGURE 5. An oblique upstream view from the surface of steady-state coarse-rich front established in the moving-bed flume of figure 3. The large blue and white marbles congregate towards the front of the picture, with the smaller clear glass beads towards the rear.

corresponds to a small or large particle. The large size ratio between the grains minimised identification errors, although there was a small possibility that a large particle may be mistaken for a small particle. This, however, would only happen if the particle was sliced close to its edge and never moved closer to the plane of the laser. A typical snapshot of the particle motion is shown in figure 6(a), where it can be seen that there are a few large particles in regions of many small particles at the upstream (left) end of the flow. These large particles are seen to move very slowly, compared with the majority of the large particles which recirculate very quickly towards the front. Figure $6(c)$ shows a time-averaged concentration plot, which was averaged over a $40 \mathrm{~min}$ period, with 1 image taken every 2 s. The slow movement of the large particles through the upstream region of small particles lowers the concentration there, and causes the 'white' 'tail'-like region.

It is worthwhile considering what influence the interstitial fluid has on the particle behaviour. The presence of a fluid (rather than air) not only modifies the interstitial pore pressures, but also couples the stress carried by the particles to that carried by the fluid flowing through gaps in the grain matrix (Iverson \& LaHusen 1989; Iverson 1997, 2005). This coupling is particularly significant in unsteady flows, since local changes in the particle volume fraction allow large excess pore pressures to develop, which in turn feedback on the granular motion (du Pont et al. 2003; Muite, Hunt \& Joseph 2004; Pailha, Nicolas \& Pouliquen 2008; Pailha \& Pouliquen 2009). However, for steady, dense granular flows such as those sketched in figure 2, the large number of particle-particle contacts mean that frictional interactions are still dominant in 
(a)

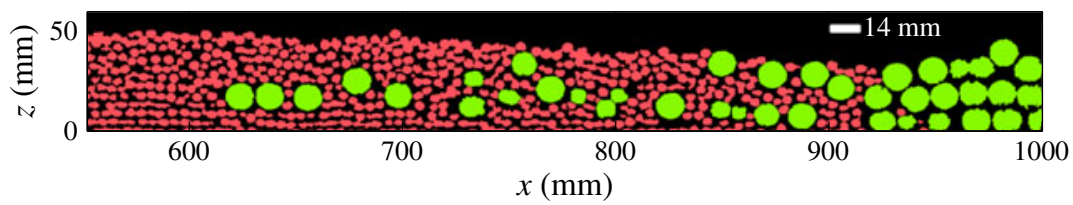

(b)

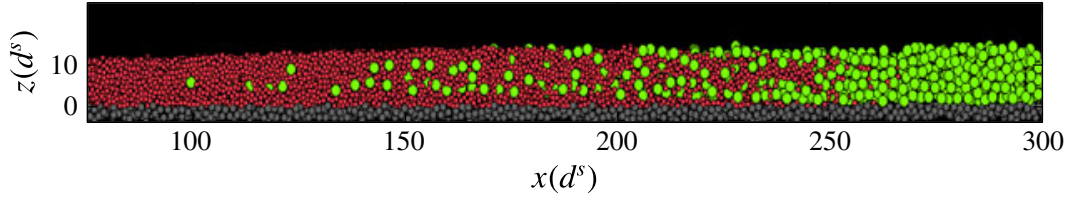

(c)

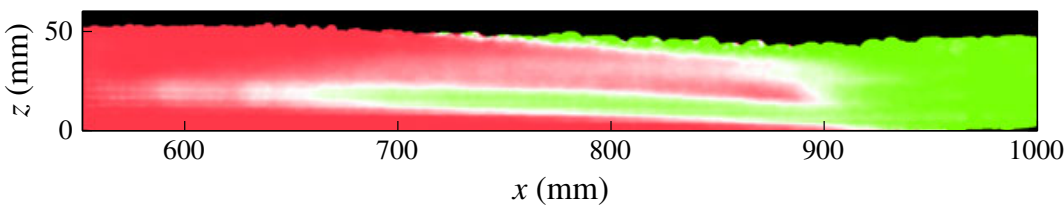

(d)

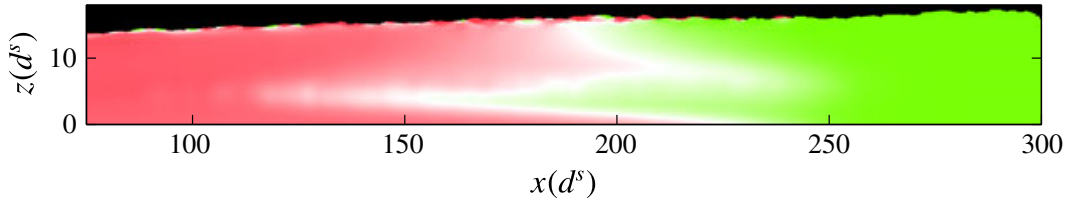

(e)

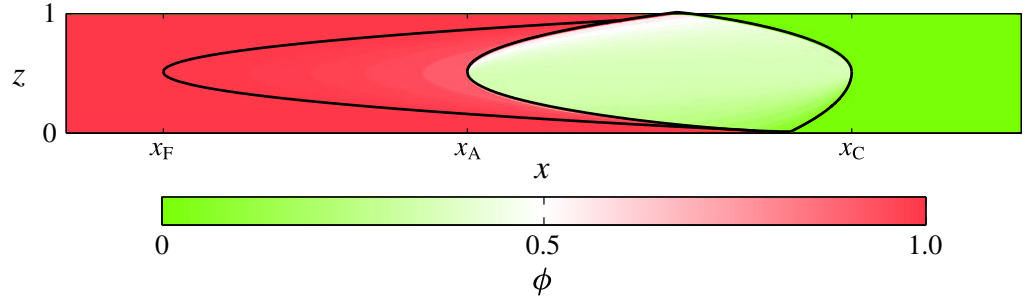

FIGURE 6. (a) An experimental snapshot of the recirculation zone, captured using the moving-bed flume of figure 3 with refractive index matched scanning. The white label indicates the length scale of $14 \mathrm{~mm}$. (b) Structure of the recirculation zone found using DPM simulations. The fixed base particles are shown in grey. Both the experimental and simulation results show several large particles positioned towards the rear, where they are surrounded by many small particles. These large particles are seen to move very slowly, and take a long time to recirculate. (c) Shows the experimental time-averaged concentration field, which was produced by averaging the individual particle positions over a $40 \mathrm{~min}$ period, with 1 image every $2 \mathrm{~s}$. The time-averaged concentration field for the simulations was produced by coarse graining all of the particle positions from 749 subsequent time frames, and is shown in $(d)$. Both of the time-averaged concentration plots indicate a 'tail' upstream, where the concentration is lower due to the slow motion of a few large grains. This is similar to asymmetric behaviour observed within a linear shear cell (van der Vaart et al. 2015), and motivates a continuum breaking wave structure with an asymmetric flux function, shown in $(e)$ for a cubic flux. The solid lines mark the boundaries of the recirculation zone, with two distinct 'lens' and 'tail' regions (see §2). The downstream 'lens' region with a strong green hue is where most of the large particles recirculate, whilst the red hue of the upstream 'tail' region shows how only a few large particles recirculate through that area. The theory does not account for spatial velocity variations, diffusive remixing or differential particle friction, and finite-size effects are also significant. These may all contribute to the difference in the 'tail' structure between the theory and the experiments and simulations. Without calibrating the segregation flux for this particular flow regime, it is remarkable that the asymmetric flux produces a 'tail' region, and it is of interest to further understand the asymmetric breaking-wave structure and particle recirculation within it. In all of the above plots, the lower belt moves from right to left, with gravity acting to cause particles to flow downstream towards the right. 
determining the rheological behaviour (Ancey, Coussot \& Evesque 1999) even when an interstitial fluid is present. Cassar, Nicolas \& Pouliquen (2005) showed that, in steady flows submerged in water, at least $75 \%$ of the overburden pressure is borne by the contact network. They also showed that the same rheology used to describe dense steady aerial flows (GDR Midi 2004) also applies to immersed flows, with the interstitial fluid changing the time scale of the particle rearrangements. This is consistent with the experimental results of Vallance \& Savage (2000) and the theory of Thornton, Gray \& Hogg (2006) who both showed that the role of the interstitial fluid in flows containing different sized constituents is to modify the segregation time scales. These results would suggest that the physical phenomena observed in the experiments above, with a few large particles recirculating very slowly in regions of small particles, are indicative an underlying asymmetry in the particle motion that occurs whether the flow is dry or submerged. Further experimental work, using techniques such as X-ray tomography (e.g. McDonald, Harris \& Withers 2012), is needed to compare the particle scale dynamics in dry flows with those containing an interstitial fluid.

Discrete particle method (DPM) simulations of a moving bed-flume set-up were also performed using the MercuryDPM code (MercuryDPM.org; Thornton et al. 2013a,b). A dry bidisperse mixture of spherical particles was used, with all of the particles of the same (non-dimensional) density $\rho^{*}=\pi / 6$, but of two different (non-dimensional) diameters, $d^{s}=1$ and $d^{l}=2.4$, for small and large particles, respectively. All of the simulation parameters were non-dimensionalised so that $g=1$. A frictional springdashpot model (Cundall \& Strack 1979; Weinhart et al. 2012) with linear elastic and linear dissipative contributions was used for both the normal and tangential forces. The tangential force models the effects of particle surface roughness, and its spring stiffness was taken to be $2 / 7$ of the spring stiffness for the normal direction. The tangential force also truncates so that it is always less than $1 / 2$ of the normal force. The particles all had the same coefficient of restitution $r_{c}=0.1538$, which was chosen to be less than typical known values for glass $(\sim 0.9)$ in order to model the dissipative effects of the interstitial fluid removing energy from the system. The contact time for all head on collisions was fixed at 0.0054 , with the collision properties chosen to be different for small/small, small/large and large/large collisions so that both the contact time and the coefficient of restitution were the same even in the mixed case. Further details of the precise DPM implementation may be found in Thornton et al. (2012b) and Weinhart et al. (2012). The simulations were conducted in a box of length $300 d^{s}$ with fixed end walls and width $8.4 d^{s}$. The side walls were periodic in order to bring the simulations closer to the assumptions of the analytic model in figure $2(b)$, which is two-dimensional and has no side-wall effects. A small inclined wall was placed between the base and the vertical upstream wall in order to prevent small particles being crushed by the wall or shooting away from it. This was seen to only affect the dynamics very close to the wall, and did not affect the recirculation zone. A rough moving base was created in several steps. Firstly, particles of diameter $d^{b}=$ 1.7 were stuck randomly to a horizontal plate. Particles of diameter $d^{b}$ were slowly dropped onto this plate and allowed to settle. Once a thick layer of height $12 d^{b}$ was produced, a slice of particles was taken whose centres lay between $9.3 d^{b}$ and $11 d^{b}$. These particles were endowed with infinite mass and inclined at an angle of $23^{\circ}$ to form the base for the moving-bed flume simulations. The layer is thick enough to ensure that no flowing particles can fall through the rough base during the simulations. More details of this base creation process can be found in Weinhart et al. (2012) whereas a detailed description of different bed creation methods and their effect on the 
macroscopic friction experienced by the flow can be found in Thornton et al. (2012a). Before each time step $\Delta t=10^{-4} \sqrt{d^{s} / g}$, the base was moved upstream by a distance $u_{\text {belt }} \Delta t=1.5 \times 10^{-4} d^{s}$. The system was allowed to evolve until a steady recirculation zone was formed.

Figure $6(b)$ shows a snapshot from the simulations, which have a very similar structure to the experimental results: most large particles recirculate quickly at the front but a few large particles recirculate slowly at the rear. This behaviour is also evident in the time- and width-averaged concentration plot shown in figure $6(d)$, which was produced by employing the micro-macro coarse-graining technique (Goldhirsch 2010; Weinhart et al. 2013) on the individual particle positions from 749 subsequent time steps. The new extension by Tunuguntla, Thornton \& Weinhart (2015), based on a mixture theory formulation (Morland 1992), allowed the (partial) densities for the bulk $(\rho)$, small $\left(\rho^{s}\right)$ and large particles $\left(\rho^{l}\right)$ to be separately extracted, with the small particle concentration defined as $\rho^{s} / \rho$, i.e. the local small particle material density over the local granular material density. The coarse-graining method used two-dimensional Gaussian functions at each of the particle positions and generated the continuum field at every point in space; however, for ease of computing, the data is shown on a $250 \times 250$ grid. As was seen in the experimental concentration field in figure $6(c)$, the slow moving large particles have lowered the upstream concentration and produced a white 'tail' protruding backwards from the main region of recirculation. This qualitative similarity between the concentration field of the simulations that were laterally periodic (figure $6 d$ ) and the concentration field of the experiments (figure $6 c$ ) indicates that there are only minimal effects arising from the side walls and justifies the two-dimensional approximation of the analytic solution. Dry simulations, using a much higher restitution coefficient, also gave a similar concentration field, indicating that the behaviour is not an artefact of the presence of the fluid nor the exact particle properties. Despite the fact that no attempt was made to calibrate the simulations and experiments, both show very similar behaviour using different sized particles in different sized flumes. The presence of the 'tail', in which large particles recirculate very slowly through regions of many small particles, points towards a fundamental asymmetry in the interactions between the large and small particles. Recently, van der Vaart et al. (2015) uncovered a similar asymmetry in a linear shear cell, and showed how the asymmetry could be modelled using a continuum approach.

\subsection{Continuum segregation equation for bidisperse mixtures}

Non-dimensional continuum models for segregation in bidisperse mixtures (e.g. Bridgwater, Foo \& Stephens 1985; Savage \& Lun 1988; Bridgwater 1994; Dolgunin \& Ukolov 1995; Gray \& Thornton 2005; Gray \& Chugunov 2006; Thornton et al. 2006; May, Shearer \& Daniels 2010) all share a similar advection-diffusion structure

$$
\frac{\partial \phi}{\partial t}+\nabla \cdot(\phi \boldsymbol{u})-\frac{\partial}{\partial z}\left(S_{r} F(\phi)\right)=\frac{\partial}{\partial z}\left(D_{r} \frac{\partial \phi}{\partial z}\right),
$$

where the $z$ coordinate is the upward pointing normal to the flume bed, the $x$ coordinate points down the flume and the $y$ coordinate points horizontally across the flume bed. The bulk velocity field $\boldsymbol{u}=(u, v, w)$ has components in the above directions, the small particle concentration is $\phi$, and $S_{r}$ and $D_{r}$ are the non-dimensional segregation and diffusive-remixing coefficients, respectively. As the typical length 
and height of the avalanche are $L$ and $H$, and magnitudes of the downstream and segregation velocities are $U$ and $Q$, the non-dimensional segregation coefficient $S_{r}=Q L /(H U)$ represents the ratio of the typical segregation time scale $Q / H$ to the typical downstream transport time scale $U / L$. Similarly, the non-dimensional diffusion coefficient $D_{r}=D L /\left(H^{2} U\right)$ represents the ratio of the typical diffusion time scale $D / H^{2}$ to the typical downstream transport time scale $U / L$, with $D$ being the diffusivity between the two particle species. The large particle concentration is $1-\phi$ since the solids volume fraction is assumed to be uniform and constant throughout the flowing layer (Rognon et al. 2007). The first term on the left-hand side in (1.1) describes the temporal evolution, whilst the second term describes the advection with the bulk flow. The segregation is captured by the third term, with $F(\phi)$ the segregation flux and the negative sign indicating that there is a net motion of small particles downwards. The segregation flux is often assumed to be the product of the small and large particle concentrations,

$$
F(\phi)=\phi(1-\phi)
$$

and has the property that segregation ceases when the concentration reaches zero (pure large phase) or unity (pure small phase). The right-hand side of equation (1.1) reduces the sharp concentration shocks that develop between the two species, and models the diffusion of one species into the other that results from the random-walk-like behaviour of the grains. In many flows, this is small compared to the segregation (Gray \& Hutter 1997; Dasgupta \& Manna 2011; Wiederseiner et al. 2011b; Thornton et al. 2012b) and so the non-diffuse solution in which $D_{r}=0$ is a useful approximation, with (1.1) reducing to a scalar hyperbolic equation. A full review of the derivation, history and applications of (1.1) can be found in Gray, Gajjar \& Kokelaar (2015).

\subsection{Asymmetry between large and small particle motion}

Recent experiments by Golick \& Daniels (2009) and van der Vaart et al. (2015) have uncovered an underlying asymmetry in the behaviour of large and small grains during segregation, with a characteristic dependence on the local relative volume fraction of small particles. Within their annular ring shear experiments, Golick \& Daniels (2009) inferred that large particles were segregating very slowly in regions of many small particles, but were not able to further explain this observation. Using a classical linear shear cell (Bridgwater 1976) and the 'refractive index matched scanning technique' (Wiederseiner et al. 2011a; Dijksman et al. 2012), experiments by van der Vaart et al. quantified on both bulk and particle scales how large particles rise slower in regions of many small particles compared to small particles percolating down through a region of many large particles. They also showed that the large particle velocity displayed a peak at approximately $\phi=0.55$, proving that the coarse grains rise quickest as a group. Gajjar \& Gray (2014) showed that the normal constituent velocities associated with the segregation equation (1.1) are

$$
w^{l}(\phi)=w+S_{r} \frac{F(\phi)}{1-\phi}, \quad w^{s}(\phi)=w-S_{r} \frac{F(\phi)}{\phi},
$$

with both velocities uniquely determined by the geometry of the flux function $F(\phi)$ at every concentration $\phi$. The velocity of the large particles $w^{l}(\phi)(1.3 a)$ is directly proportional to the gradient of the chord, namely the gradient of the straight line segment (Clapham \& Nicholson 2009), joining $(1,0)$ with $(\phi, F(\phi))$. Similarly, the 
velocity of the small particles $w^{s}(\phi)$ is directly proportional to the gradient of the chord joining $(0,0)$ with $(\phi, F(\phi))$. A pair of these two chords for $\phi=\phi_{\max }$ are shown in figure $7(b)$. Since the quadratic segregation flux (1.2) utilised by many segregation models is symmetric about $\phi=0.5$ (figure $7 a$ ), it gives linear segregation velocities for the large and small grains

$$
w^{l}(\phi)=w+S_{r} \phi, \quad w^{s}(\phi)=w-S_{r}(1-\phi) .
$$

The maxima of these velocities are equal in magnitude (figure 7c), and so (1.2) is unable to capture the asymmetry measured by van der Vaart et al. (2015). In order to model the asymmetric behaviour between large and small grains, Gajjar \& Gray (2014) introduced a new class of flux functions with the following properties: (i) $F(\phi)$ is skewed towards $\phi=0$, with a maximum occurring at $0<\phi_{\max }<1 / 2$; (ii) $F(\phi)$ is normalised to have the same amplitude as the quadratic flux (1.2); and (iii) $F(\phi)$ has at most one inflexion point $\phi_{\text {inf }}$ in the interval $\left(\phi_{\max }, 1\right)$. Although there are other ways of normalising the class of flux functions, e.g. by the area, there were no qualitative differences between the different methods. The simplest flux function fitting all of the above requirements is the cubic form

$$
F(\phi)=A_{\gamma} \phi(1-\phi)(1-\gamma \phi),
$$

where $\gamma$ is the asymmetry parameter and $A_{\gamma}$ is a normalisation constant. Note that the limit $\gamma \rightarrow 0$ of (1.5) recovers the symmetric quadratic flux (1.2). For small amounts of asymmetry, $0 \leqslant \gamma \leqslant 0.5, F(\phi)$ is convex up (Clapham \& Nicholson 2009), whilst for greater amounts of asymmetry $0.5<\gamma \leqslant 1, F(\phi)$ is non-convex with a single inflexion point

$$
\phi_{i n f}=\frac{1+\gamma}{3 \gamma} .
$$

As shown in figure $7(c)$, the cubic functions (1.5) are able to reproduce the asymmetric behaviour that a small particle will percolate down more quickly at low $\phi$ (figure 7e) than a large particle rises upwards at high $\phi$ (figure $7 g$ ). In addition, figure $7(b)$ shows how the presence of an inflexion point (1.6) means that the chord joining $(\phi, F(\phi))$ with $(1,0)$ initially has an increasing gradient as $\phi$ increases from 0 to $\phi_{\mathrm{M}}$, and a decreasing gradient thereafter. Thus, the non-convex flux functions display a maximum in the large particle velocity at an intermediate concentration $\phi_{\mathrm{M}}$ (figure $7 f$ ). This behaviour will be known as the collective motion of the large particles.

Gajjar \& Gray (2014) were able to examine the influence of asymmetry on the segregation process by constructing exact solutions to the non-diffuse $\left(D_{r}=0\right)$ hyperbolic segregation equation (1.1) using the method of characteristics (e.g. Whitham 1974; Billingham \& King 2001). Concentration $\phi$ is constant along characteristic curves, which are also simply known as characteristics. The characteristics combine to form distinct features in the solution, such as rarefaction fans, shocks, semi-shocks and compressions, with physical definitions of these features provided in appendix A. Characteristics may diverge and form an expansion fan, with a smoothly varying concentration field, or converge and form a shock with a sharp jump in concentration from the rearward (-) side to the forward $(+)$ side. The propagation of the shock surface $z_{s}(t, x, y)$ is governed by

$$
\frac{\partial z_{s}}{\partial t}+u \frac{\partial z_{s}}{\partial x}+v \frac{\partial z_{s}}{\partial y}-w=-S_{r} \frac{\llbracket F(\phi) \rrbracket}{\llbracket \phi \rrbracket},
$$



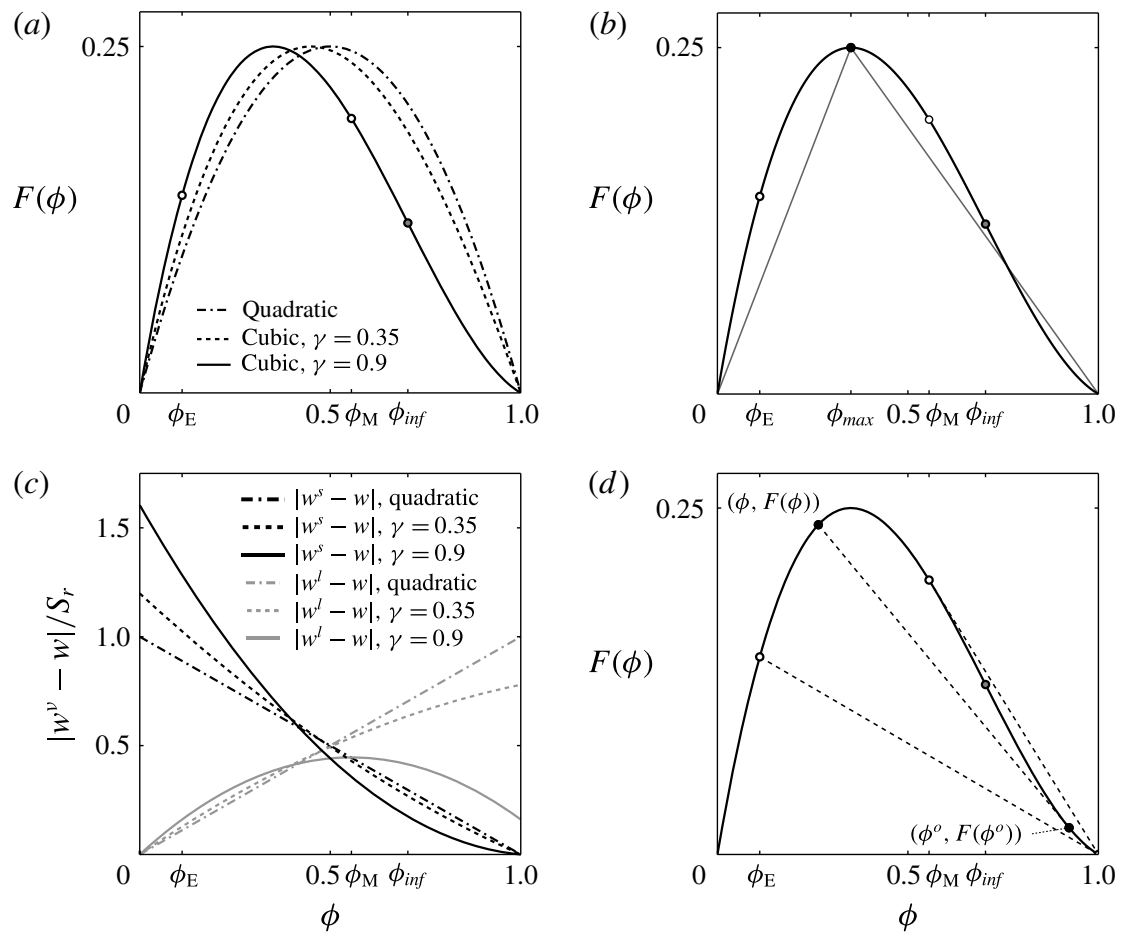

(e)

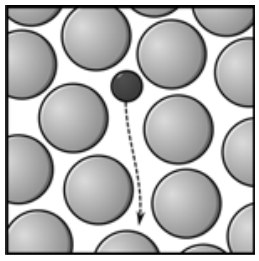

$(f)$

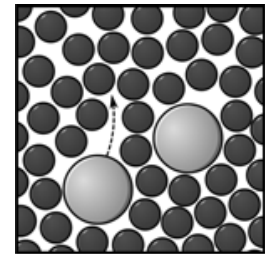

(g)

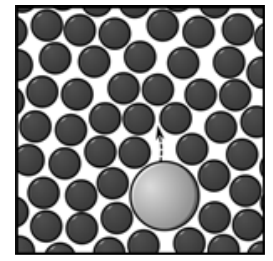

FIGURE 7. There is an intrinsic geometric relationship between the segregation flux $F(\phi)$ shown in $(a)$, and its segregation velocities $w^{v}(1.3)$ shown in (c). At any concentration $\phi$, the gradient of the chords (straight line segment) joining $(\phi, F(\phi))$ with $(1,0)$ and $(0,0)$ are proportional to the velocities (1.3) of the large and small particles, respectively. These chords are illustrated in $(b)$ for $\phi=\phi_{\max }=\phi_{\mathrm{R}}$. The quadratic flux (1.2) is symmetric about $\phi=0.5$, and thus gives linear segregation velocities (1.4) that have the same magnitude. The cubic flux is skewed towards $\phi=0$ with a maximum occurring at $0<\phi_{\max }=\phi_{\mathrm{R}}<1 / 2$, and is normalised by (2.8) to have the same amplitude as the quadratic flux. This gives asymmetric segregation velocities, with a single small particle $(e)$ having a greater velocity that a single large particle $(g)$. For higher amounts of asymmetry, measured by the asymmetry parameter $\gamma$, the cubic flux has an inflexion point at $\phi_{\text {inf }}=(1+\gamma) / 3 \gamma$. It is this inflexion point which causes the large particle velocity to have a peak at an intermediate concentration $\phi_{\mathrm{M}}$, with large particles moving quickest when in close proximity to other large particles $(f)$. $(d)$ The image point $\phi^{\circ}$ (1.8) of concentration $\phi$ is defined as the point at which the gradient of the tangent to the flux function $F^{\prime}\left(\phi^{o}\right)$ is equal to the gradient of the chord joining $\phi$ to $\phi^{o}$ on $F$. These pairs of concentrations $\left\{\phi, \phi^{o}\right\}$ (filled black circles) cause the formation of semi-shocks, where only the characteristics of concentration $\phi$ collide with shock on one side, whilst the characteristics of concentration $\phi^{o}$ lie tangential to the shock on the other side. Two pairs of concentrations $\left\{1,1^{o}=\phi_{\mathrm{M}}\right\}$, and $\left\{\phi_{\mathrm{E}}, \phi_{\mathrm{E}}^{o}=1\right\}$ (open circles) are particularly important in the solutions, with the chords tangential at $\phi=\phi_{\mathrm{M}}$ and $\phi=1$ respectively. Note that the segregation flux in $(b)$ and $(d)$ is the cubic flux (1.5) with $\gamma=0.9$. 
with 'jump' brackets $\llbracket f \rrbracket=f^{+}-f^{-}$denoting the discontinuity in $f$ across the shock (Gray, Shearer \& Thornton 2006). Note that the right-hand side of (1.7) is proportional to the gradient of the chord on flux $F(\phi)$ between $\phi=\phi^{-}$and $\phi=\phi^{+}$(Gajjar \& Gray 2014). The characteristics usually collide with both sides of a shock, but the non-convex cubic flux functions give rise to a special type of shock, known as a semi-shock (Rhee, Aris \& Amundson 1986), where characteristics only collide with one side of the shock and are tangential to it on the other. The image point $\phi^{\circ}$ of concentration $\phi$ is defined as the point at which the gradient of the tangent to the flux function $F^{\prime}\left(\phi^{o}\right)$ is equal to the gradient of the chord joining $\phi$ to $\phi^{o}$ on $F$, with the shock condition (1.7) giving the relation

$$
F^{\prime}\left(\phi^{o}\right)=\frac{F(\phi)-F\left(\phi^{o}\right)}{\phi-\phi^{o}} .
$$

By this definition, the characteristics of concentration $\phi^{o}$ lie tangential to the shock, whilst the characteristics of concentration $\phi$ collide with the other side. For the cubic flux function (1.5), the relationship (1.8) between concentrations $\phi$ and $\phi^{o}$ simplifies to

$$
\phi^{o}=\frac{1}{2}\left(\frac{1+\gamma}{\gamma}-\phi\right) .
$$

An example pair of concentrations $\left\{\phi, \phi^{\circ}\right\}$ is shown with closed black circles in figure $7(d)$. It is possible that the characteristics of concentration $\phi^{\circ}$ may collide with another semi-shock; characteristics of concentration $\left(\phi^{o}\right)^{o}=\phi^{o o}$ would lie tangential to this semi-shock on the other side. An example of the relationship between $\phi$, $\phi^{o}$ and $\phi^{o o}$ is illustrated in figure 8. Two pairs of concentrations $\left\{1,1^{\circ}=\phi_{\mathrm{M}}\right\}$, and $\left\{\phi_{\mathrm{E}}, \phi_{\mathrm{E}}^{o}=1\right\}$ are of particular importance in the exact solutions, with

$$
1^{o}=\phi_{\mathrm{M}}=\frac{1}{2 \gamma} \quad \text { and } \quad \phi_{\mathrm{E}}=\frac{1-\gamma}{\gamma},
$$

using the short hand notation $1^{\circ}=\left.\phi^{o}\right|_{\phi=1}$. As shown by the open circles in figure $7(d)$, the chord between $\left(\phi_{\mathrm{M}}, F\left(\phi_{\mathrm{M}}\right)\right)$ and $(1,0)$ is tangential to the segregation flux $F$ at $\phi=\phi_{\mathrm{M}}$, whilst the chord between $\left(\phi_{\mathrm{E}}, F\left(\phi_{\mathrm{E}}\right)\right)$ and $(1,0)$ is tangential to $F$ at $\phi=1$. Concentration $\phi_{\mathrm{M}}$ has the physical significance that it is the concentration at which the large particles reach their maximum velocity and is important in the solution structure described in $\S 2.2$, whilst concentration $\phi_{\mathrm{E}}$ is important in the structure described in $\S 2.3$, and determines which of the two non-convex solutions is formed.

Tunuguntla, Bokhove \& Thornton (2014) showed that asymmetry causes the distance for complete segregation of an initially homogeneous mixture to become dependent on the initial conditions, and Gajjar \& Gray (2014) specifically found the distance to be dependent on the inflow concentration, with a higher proportion of fines increasing the final segregation distance. In addition, the decreasing large particle velocity at higher concentrations causes semi-shocks to form, where large particles take longer to rise to the upper layer. This creates a stronger dependence of the final segregation distance on the inflow concentration for both homogeneous and normally graded inflow profiles, similar to the linear relationship reported by both Staron \& Phillips (2014) and van der Vaart et al. (2015). In particular, van der Vaart et al. (2015) were able to fit their data to a non-convex cubic flux with $\gamma=0.89$, which also matched their experimental observation of a peak in the large particle velocity around $\phi=0.55$. It is also interesting that asymmetric segregation flux functions arise naturally in the work of Gray \& Ancey (2015), which extends the model of Gray \& Chugunov (2006) to account for differences in both particle size and particle density. 


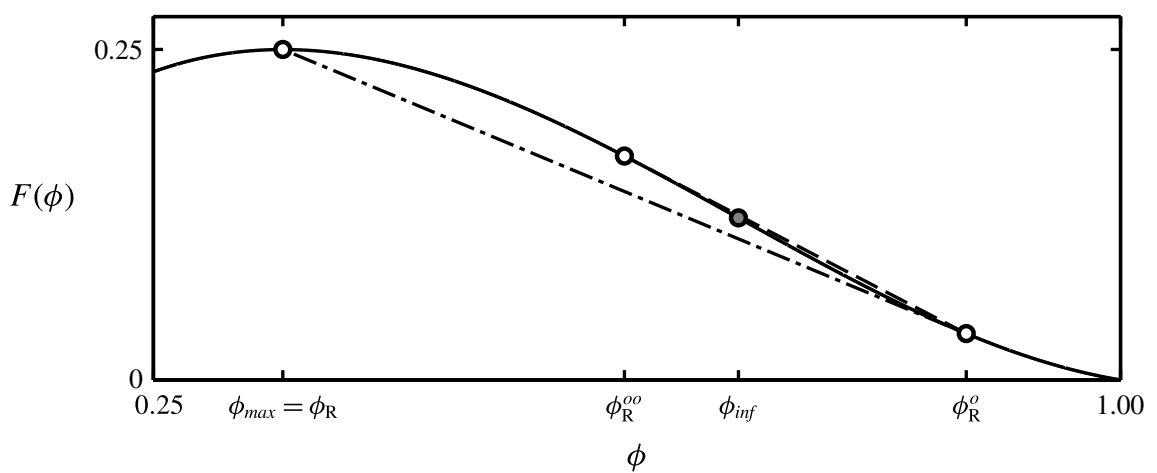

FIGURE 8. A sketch showing the relationship between $\phi_{\mathrm{R}}, \phi_{\mathrm{R}}^{o}$ and $\left(\phi_{\mathrm{R}}^{o}\right)^{o}=\phi_{\mathrm{R}}^{o o}$ for the cubic flux with $\gamma=0.9$ (see (1.5)). The dash-dotted line shows that the chord joining $\phi_{\mathrm{R}}$ to $\phi_{\mathrm{R}}^{o}$ is tangential to the flux function at $\phi_{\mathrm{R}}^{o}$, whilst the dashed line shows that the chord joining $\phi_{\mathrm{R}}^{o}$ with $\phi_{\mathrm{R}}^{o o}$ is tangential to the flux function at $\phi_{\mathrm{R}}^{o o}$. These points are important in the construction of the 'lens-tail' structure in $\S 2.3$.

\subsection{Breaking size-segregation waves}

One of the strengths of the continuum theory is its ability to reveal the structure and development of the recirculation zone that plays a vital role in the formation of bouldery fronts (Thornton \& Gray 2008; Gray \& Ancey 2009; Johnson et al. 2012). The simplest recirculation structure arises in the case of steady uniform flow (Pouliquen 1999b; Rognon et al. 2007; Forterre \& Pouliquen 2008), in which the flow thickness $h$ is constant. The combination of the propensity of the avalanche to form an upward coarsening size distribution through particle size segregation and the shear profile

$$
\boldsymbol{u}=(u(z), 0,0)
$$

means that a monotonically decreasing interface separating large particles above from small particles below (figure 9a) will continually steepen as fine grains are sheared over the top of coarse grains (figure $9 b$ ). The interface eventually breaks in finite time (figure 9c, Gray et al. 2006), forming a recirculation zone (figure $9 d$ ) in which the large grains lying immediately below small grains are resegregated back towards the surface, and then swept downstream by the shear velocity (Thornton \& Gray 2008; Gray \& Kokelaar 2010a,b). The similarity with classical breaking waves formed when an air-water interface steepens and breaks (Basco 1985; Shand 2009) led Thornton \& Gray (2008) to refer to the propagating recirculation zone as a breaking size-segregation wave.

The bulk velocity field (1.11) implies that the segregation equation (1.1) reduces to

$$
\frac{\partial \phi}{\partial t}+\frac{\partial}{\partial x}(\phi u(z))-\frac{\partial}{\partial z}\left(S_{r} F(\phi)\right)=0 .
$$

Numerical solutions to (1.12) using a simple TVD Lax-Friedrichs shock-capturing finite volume scheme (Yee 1989; Tóth \& Odstrčil 1996; LeVeque 2002) show that the breaking size-segregation wave initially has a complex structure (figure $9 d$ ) that oscillates back and forth in time like a spinning rugby ball (Thornton \& Gray 2008). Exact solutions for the structure have only been derived for the early stages of wave 


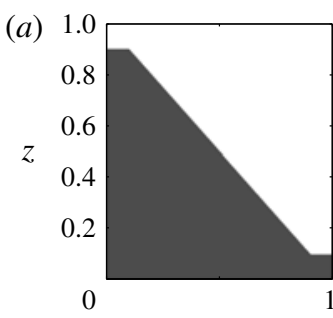

$x$

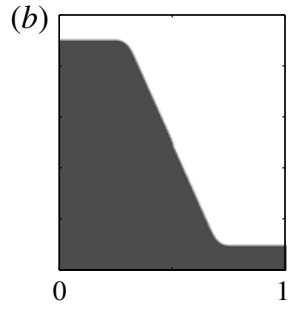

$x$ $(c)$

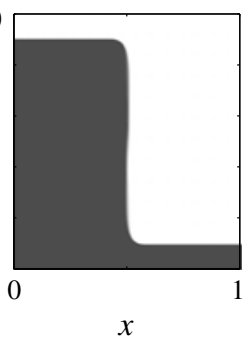

$(d)$

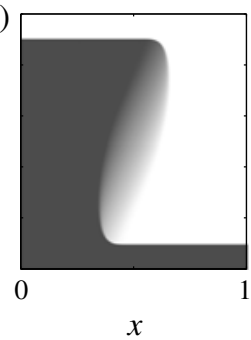

(e)
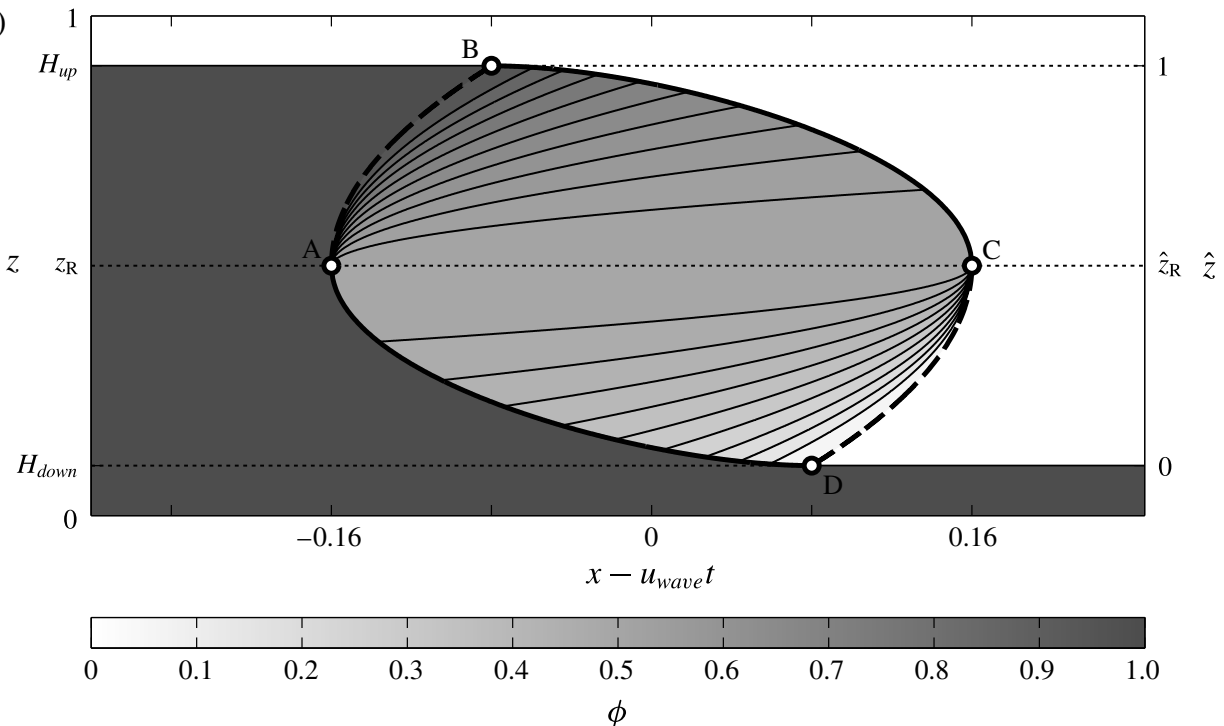

FIGURE 9. Numerical solutions of the segregation equation (1.12) in a steady uniform flow with a quadratic flux (1.2) show that a monotonically decreasing interface between large and small grains $(a)$ continually steepens in time $(t=0.0)(b)$ as small particles are sheared over the top of large particles $(t=0.5)$. This interface breaks in finite time $(t=1.0)(c)$ and forms a recirculation zone $(t=1.5)(d)$, in which the large particles rise upwards towards the surface as they are resegregated before being sheared back towards the front. The recirculating zone has a complex 'breaking-wave' structure that oscillates in time, however the oscillations exponentially decay and the structure tends towards a steady state. (e) The steady breaking wave (Thornton \& Gray 2008) for the quadratic flux function (1.2) exists between the vertical heights $H_{\text {down }}=0.1$ and $H_{u p}=0.9$, and consists of two expansion fans and two concentration shocks arranged in a 'lens'-like structure. The two expansion fans are ABCA centred at point $\mathrm{A}$ and $\mathrm{CDAC}$ centred at point $\mathrm{C}$, with individual characteristic curves shown with thin solid lines. The edge of the expansion fans are the $\phi=1$ and $\phi=0$ characteristics, which lie along $\mathrm{AB}$ and $\mathrm{CD}$, respectively, and are shown with thick dashed lines. The two shocks are BC and DA, and are shown with thick solid lines. However, this structure is unable to replicate the slow movement of large particles upstream of the main recirculation region that was seen in figure 6 .

breaking (McIntyre et al. 2007), however, the simulations show that oscillations are transient and exponentially decay, with the structure tending towards a steady state. Thornton \& Gray (2008) generated an exact solution for the steady wave with the quadratic flux (1.2). As shown in figure $9(e)$, it consists of two expansion fans and two concentration shocks arranged in a 'lens'-like structure. In general, the breaking 
wave forms between the two vertical heights $z=H_{\text {down }}$ and $z=H_{u p}$, and propagates at a speed $u_{\text {wave }}$ that is equal to the mean speed between these heights,

$$
u_{\text {wave }}=\frac{1}{H_{u p}-H_{\text {down }}} \int_{H_{\text {down }}}^{H_{u p}} u(z) \mathrm{d} z .
$$

Note that the recirculation zone within the moving-belt flume in $\S 1.1$ occupies the entire height, hence $H_{\text {down }}=0$ and $H_{u p}=1$. Since the velocity $u(z)$ is monotonically increasing, the breaking wave propagates faster than the basal velocity but slower than both the surface velocity and the front velocity $u_{\text {front }}$ (Gray \& Ancey 2009). At a height $z=z_{\mathrm{R}}$, the bulk velocity is equal to $u_{\text {wave }}$. Above $z_{\mathrm{R}}, u(z)>u_{\text {wave }}$, and so material is swept towards the breaking wave from the left, whilst for $z<z_{\mathrm{R}}$, $u(z)<u_{\text {wave }}$ and so material flows towards the breaking wave from the right. The change in flow direction relative to the 'lens' at $z=z_{\mathrm{R}}$ is crucial, and thus both expansion fans are initiated at this height, centred at points $\mathrm{A}$ and $\mathrm{C}$. The $\phi=1$ characteristic lies between points $\mathrm{A}$ and $\mathrm{B}$, whilst the $\phi=0$ characteristic lies between points $\mathrm{C}$ and $\mathrm{D}$. Two concentration shocks join point $\mathrm{B}$ with $\mathrm{C}$ and point $\mathrm{D}$ with A, respectively. Although the upper portion of the 'lens' ABCA contains lower concentrations than the lower portion of the 'lens' CDAC, the positions of the characteristics, expansion fans and shocks are rotationally invariant about the centre of the lens. This is a direct result of the symmetry of the quadratic flux (1.2) about $\phi=0.5$.

Gray \& Ancey (2009) derived the structure of the steady-state recirculation zone in a non-uniform depositing flow that was reconstructed from a travelling wave solution to the depth-averaged avalanche equations (Savage \& Hutter 1989; Pouliquen 1999a,b; Wieland, Gray \& Hutter 1999; Gray, Tai \& Noelle 2003). They found that the breaking wave also consisted of two expansion fans and two shocks arranged in a 'lens', but surrounding a central 'eye' of constant concentration. The wave is located at a unique position behind the flow front and determines the concentration deposited within the basal layer. The model was able to qualitatively describe the features of their experimental two-dimensional depositing flow constrained by lateral side walls, namely the coarse-grained flow front, the rapidly moving large particles on the surface and the static layer of coarse grains at the base sandwiching an intermediate layer of fine grains. The experiments, were, however, too grainy to resolve the finer structure of the breaking wave.

In the absence of the two-dimensional side-wall restrictions, Johnson et al. (2012) numerically solved for the structure of the recirculation zone on the centreline of a three-dimensional front, which has a more elaborate 'breaking-wave structure'. Numerical solutions suggest that both the characteristic curves and the particle paths continually spiral inwards, because of the sidewards advection of mass into the lateral levees. The exact analytic structure of the three-dimensional recirculation zone is still proving illusive.

Figure 6(e) shows a breaking-wave structure using the simple asymmetric cubic model (1.5) from $\S 1.3$. As with the structure of the symmetric flux in figure 9, the asymmetric wave also has a 'lens'-like structure towards the downstream end. The asymmetry also causes a new upstream 'tail' to be produced, through which a few large particles recirculate slowly. Although this behaviour is very similar to the individual particle motion observed in experiments and simulations in $\S 1.1$, the shape and structure of the 'tail' region are qualitatively different. There are a number of other factors present within the moving-bed flume set-up used in both the experiments 
and simulations that are unaccounted for by the simple theory. Streamwise spatial variations in the velocity field, diffusive remixing and the differential friction of the two particles on the moving base may all have an influence on the 'tail' shape. The size of the system and finite-size effects may also contribute to the discrepancy in the 'tail' structure. Further experimental work and extensive simulations are currently being conducted in order to understand more about the slow particle movement through the 'tail'. Nevertheless, without any knowledge of the exact shape of the segregation flux function in this environment (Gajjar \& Gray 2014), the fact that a simple asymmetric cubic flux produces a 'tail' means that it is of interest to understand the derivation and particle paths. This paper examines the effect of an asymmetric segregation flux function (Gajjar \& Gray 2014; van der Vaart et al. 2015) on both the structure of a two-dimensional breaking size-segregation wave, and the particle recirculation within it.

\section{Steady-state structure of the travelling breaking wave}

The simplest steady-state breaking wave occurs under steady uniform flow $(\S 1.4)$, and exists between the vertical heights $z=H_{\text {down }}$ and $z=H_{u p}$. The wave propagates forwards with velocity $u_{\text {wave }}$, and it is convenient to transfer to a (Lagrangian) reference frame translating with the recirculation zone by employing the change of variables

$$
\hat{t}=\frac{S_{r}}{H_{u p}-H_{\text {down }}} t, \quad \hat{x}=\frac{S_{r}}{H_{u p}-H_{\text {down }}}\left(x-u_{\text {wave }} t\right), \quad \hat{z}=\frac{z-H_{\text {down }}}{H_{\text {up }}-H_{\text {down }}} . \quad(2.1 a-c)
$$

At steady state, the wave is stationary in this frame. The wave has also conveniently been stretched to lie between $\hat{z}=0$ and $\hat{z}=1$, whilst the $S_{r}$ parameter dependence has been removed. The segregation equation (1.12) becomes a simple quasi-linear equation

$$
\hat{u} \frac{\partial \phi}{\partial \hat{x}}-\frac{\partial}{\partial \hat{z}} F(\phi)=0
$$

where the relative velocity $\hat{u}=u-u_{\text {wave }}$. Equations (1.11) and (2.1) also simplify the shock condition (1.7) to give

$$
\hat{u} \frac{\partial \hat{z}_{s}}{\partial \hat{x}}=-\frac{\llbracket F(\phi) \rrbracket}{\llbracket \phi \rrbracket} .
$$

Equation (2.2) may be solved using the method of characteristics (e.g. Whitham 1974). The analysis is simplified by mapping to velocity-integrated coordinates $(\xi, \psi)$

$$
\xi=\hat{x}, \quad \psi(\hat{z})=\int_{0}^{\hat{z}} \hat{u}\left(\hat{z}^{\prime}\right) \mathrm{d} \hat{z}^{\prime} .
$$

Under this transformation, (2.2) becomes

$$
\frac{\partial \phi}{\partial \xi}-\frac{\partial}{\partial \psi} F(\phi)=0
$$

with the concentration $\phi$ taking the constant value $\phi_{\lambda}$ on straight line characteristics of gradient

$$
\frac{\partial \psi}{\partial \xi}=-F^{\prime}\left(\phi_{\lambda}\right)=-A_{\gamma}\left(3 \gamma \phi_{\lambda}^{2}-2(1+\gamma) \phi_{\lambda}+1\right) .
$$


The shock condition (2.3) also reduces to

$$
\frac{\mathrm{d} \psi}{\mathrm{d} \xi}=-\frac{\llbracket F(\phi) \rrbracket}{\llbracket \phi \rrbracket} .
$$

Transformation (2.4) splits the domain into two sections, which are separated by the no-mean-flow line $\hat{z}=\hat{z}_{\mathrm{R}}$. In the lower domain, $\psi$ decreases from $\psi=0$ at $z=0$ to $\psi=\psi_{\mathrm{R}}<0$ at $\hat{z}=\hat{z}_{\mathrm{R}}$, with both the bulk flow and time-like direction to the left, whilst in the upper domain $\psi$ increases from $\psi=\psi_{\mathrm{R}}$ at $\hat{z}=\hat{z}_{\mathrm{R}}$ to $\psi=0$ at $\hat{z}=1$, with the bulk flow and time-like direction to the right. The characteristics in each domain can be calculated independently, with the concentrations matched across $\hat{z}=\hat{z}_{\mathrm{R}}$.

For the cubic flux (1.5), the characteristics form three distinct breaking-wave structures for different values of asymmetry parameter $\gamma$, as shown in figure 10 . A 'lens'-like structure (figure 10a) that is very similar to that of Thornton \& Gray (2008) is formed for convex flux functions with low amounts of asymmetry $(\gamma \leqslant 0.5)$. The only differences between the two structures are that the top of the convex 'lens' is shifted to the right because of large particles rising at a slower rate than the percolating fines, and that the structures are no longer rotationally invariant. The symmetric structure of Thornton \& Gray (2008) is, however, recovered in the limit $\gamma \rightarrow 0$. The new 'lens' structure derivation presented here is implicit in terms of the small particle concentration $\phi$, and so is valid for not only the quadratic (1.2) and cubic fluxes (1.5), but also other convex asymmetric flux functions such as those of Marks, Rognon \& Einav (2012) and Tunuguntla et al. (2014). A second 'lens'-like structure (figure 10b) is formed for non-convex flux functions with low amounts of asymmetry $(0.5<\gamma \leqslant \Gamma$ where $\Gamma=(5+\sqrt{5}) / 10)$. The top of the 'lens' is shifted further to the right as compared to the convex lens, and an additional semi-shock is found in the upper region. A new 'lens-tail' structure (figure 10c) arises for larger amounts of asymmetry $(\Gamma<\gamma \leqslant 1)$. There is a large difference between the speeds of large and small particles, and additionally collective motion is observed, where large particles preferentially rise together in a group (van der Vaart et al. 2015). These combine to produce an additional 'tail'-like region to the left of the 'lens' where a few large particles rise very slowly and are swept a long way downstream. Each of these structures is examined in more detail below.

\subsection{Convex 'lens' structure}

First consider the 'lens' structure of the convex flux when $\gamma \leqslant 0.5$. The 'lens' is formed from two shocks $\mathrm{BC}$ and $\mathrm{DA}$ and two expansion fans $\mathrm{ABCA}$ and CDAC, as shown in figure $10(a)$ for $\gamma=0.35$. The front of the breaking wave is positioned at $\xi_{\mathrm{C}}$, and as $F^{\prime}\left(\phi_{\max }\right)=0$, the $\phi=\phi_{\max }$ characteristic is horizontal along the no-mean-flow line $\hat{z}=\hat{z}_{\mathrm{R}}$. Concentration $\phi_{\max }$ will thus be known as $\phi_{\mathrm{R}}$ throughout the remainder of this paper. Note that the definition of the asymmetric flux function in $\S 1.3$ implies that

$$
F\left(\phi_{\mathrm{R}}\right)=1 / 4
$$

Within the lower domain $\hat{z}<\hat{z}_{\mathrm{R}}$, rarefaction fan CDAC is centred at point $\mathrm{C}$ with concentrations in the range $\left[0, \phi_{\mathrm{R}}\right]$. From (2.6), each characteristic of the rarefaction fan is given by

$$
\psi=\psi_{\mathrm{R}}-F^{\prime}(\phi)\left(\xi-\xi_{\mathrm{C}}\right)
$$



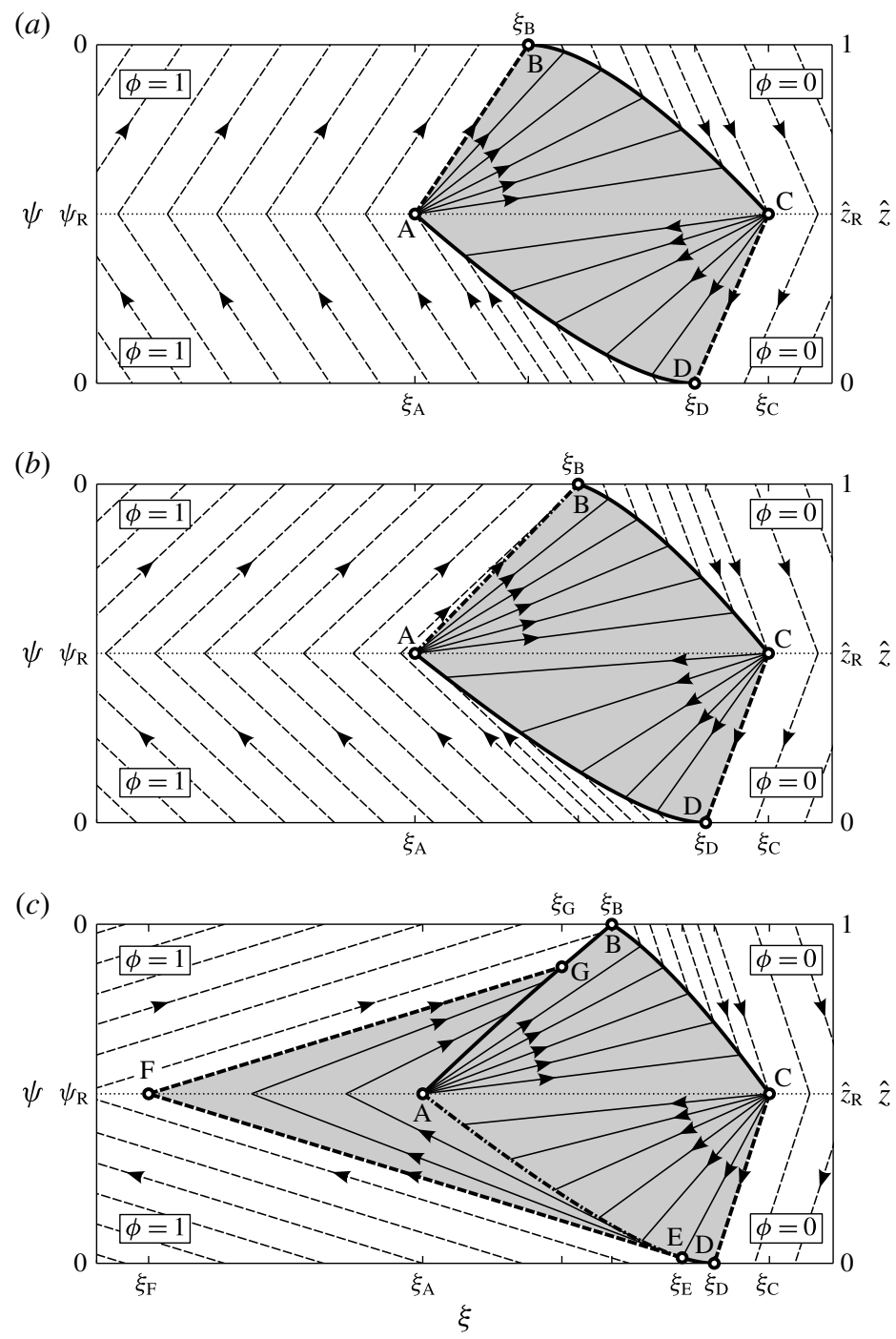

FIGURE 10. Schematic diagrams of the exact solutions to illustrate the breaking-wave structures. The characteristic curves are shown in transformed coordinates $(\xi, \psi)$, with transformation (2.4) splitting the domain into two regions separated by the no-mean-flow line $\hat{z}=\hat{z}_{R}, \psi=\psi_{R}$. In the lower region $\left(\hat{z}<\hat{z}_{\mathrm{R}}\right)$, the bulk flow and the time-like direction are both to the left, whilst in the upper region $\left(\hat{z}>\hat{z}_{R}\right)$, they are both to the right. Three different breaking-wave structures are formed for different values of the asymmetry parameter $\gamma$. A 'lens'-like structure is formed for both convex flux functions, $0<\gamma \leqslant 0.5$, and non-convex flux functions with $0.5<\gamma \leqslant \Gamma$, as shown for $\gamma=0.35$ and $\gamma=0.65$ in $(a)$ and $(b)$, respectively. The difference between the two is that the outer characteristic of the rarefaction fan $\mathrm{AB}$ becomes a semi-shock with non-convex flux functions in $(b)$. A 'lens-tail' structure is formed for higher values of asymmetry, $\Gamma<\gamma \leqslant 1$, as shown for $\gamma=0.9$ in $(c)$. The characteristics of the pure phases of large and small particles are shown with thin dashed straight lines, whilst the characteristics within the breaking wave are shown with thin solid straight lines. Thick solid lines indicate shocks, thick dash-dot lines represent a semi-shock whilst thick dashed straight lines mark the edge of an expansion fan or compression wave. None of the above structures with $\gamma>0$ have rotational symmetry about the centre of the lens. Contoured plots of these solutions are shown in figure 12 , in physical $(x, z)$ coordinates. 
The $\phi=0$ characteristic CD separates the breaking wave from the region of large particles downstream, and reaches the bottom of the wave at point $\mathrm{D}$, where $\psi=0$ and $\xi=\xi_{\mathrm{D}}$

$$
\xi_{\mathrm{D}}=\xi_{\mathrm{C}}+\frac{\psi_{\mathrm{R}}}{F^{\prime}(0)} .
$$

A sharp concentration shock DA separates the breaking wave $\left(\phi^{-}=\phi\right)$ from the upstream region of small particles $\left(\phi^{+}=1\right)$, with gradient given by $(2.7)$

$$
\frac{\mathrm{d} \psi}{\mathrm{d} \xi}=\frac{F(\phi)}{1-\phi} .
$$

Following Gajjar \& Gray (2014), a differential equation governing the downstream position of shock DA may be derived in terms of the small particle concentration $\phi$. Using the chain rule, the shock gradient (2.11) may be written as

$$
\frac{\mathrm{d} \psi}{\mathrm{d} \phi}=\frac{F(\phi)}{1-\phi} \frac{\mathrm{d} \xi}{\mathrm{d} \phi} .
$$

The rarefaction characteristics (2.9) which govern the concentration on the lower side of the shock $\left(\phi^{-}=\phi\right)$ may be differentiated with respect to $\phi$ to give

$$
\frac{\mathrm{d} \psi}{\mathrm{d} \phi}=-F^{\prime \prime}(\phi)\left(\xi-\xi_{\mathrm{C}}\right)-F^{\prime}(\phi) \frac{\mathrm{d}}{\mathrm{d} \phi}\left(\xi-\xi_{\mathrm{C}}\right) .
$$

Equating (2.12) and (2.13) yields an ordinary differential equation (ODE) for the shock path DA, which may be written as

$$
\frac{\mathrm{d}}{\mathrm{d} \phi}\left[\left(F(\phi)+(1-\phi) F^{\prime}(\phi)\right)\left(\xi-\xi_{\mathrm{C}}\right)\right]=0 .
$$

The above sequence of steps to combine (2.9) and (2.11) into (2.14) is important and will be used throughout this paper to derive equations for shocks and particle paths. Shock DA starts from point D where $\phi=0$, and so (2.14) can be integrated to give the implicit position of the shock as

$$
\xi=\xi_{\mathrm{C}}+\frac{\psi_{\mathrm{R}}}{F(\phi)+(1-\phi) F^{\prime}(\phi)},
$$

where the concentration $\phi \in\left[0, \phi_{R}\right]$ in the rarefaction fan is used to parametrise the shock path, and the height $\psi=\psi(\phi, \xi)$ is given by (2.9). When $\phi=\phi_{\mathrm{R}}$, shock DA meets the no-mean-velocity line $\hat{z}=\hat{z}_{\mathrm{R}}$ at point $\mathrm{A}$, where $\psi=\psi_{\mathrm{R}}$ and $\xi=\xi_{\mathrm{A}}$

$$
\xi_{\mathrm{A}}=\xi_{\mathrm{C}}+\frac{\psi_{\mathrm{R}}}{F\left(\phi_{\mathrm{R}}\right)} .
$$

There is also a rarefaction fan ABCA centred at point $\mathrm{A}$ in the upper domain $\left(\hat{z}>\hat{z}_{\mathrm{R}}\right)$, with characteristics

$$
\psi=\psi_{\mathrm{R}}-F^{\prime}(\phi)\left(\xi-\xi_{\mathrm{A}}\right),
$$


for $\phi \in\left[\phi_{\mathrm{R}}, 1\right]$. The $\phi=1$ characteristic $\mathrm{AB}$ separates the left-hand edge of the breaking wave from the small particle region upstream and reaches the top at point $\mathrm{B}$, where $\psi=0$ and

$$
\xi=\xi_{\mathrm{B}}=\xi_{\mathrm{A}}+\frac{\psi_{\mathrm{R}}}{F^{\prime}(1)} .
$$

Shock BC exists between points $\mathrm{B}$ and $\mathrm{C}$, and separates the rarefaction fan characteristics within the breaking wave $\left(\phi^{-}=\phi\right)$ from the pure large particle phase downstream $\left(\phi^{+}=0\right)$. Combining (2.7) and (2.17) in the same manner as (2.9) and (2.11) above yields the governing differential equation for the streamwise shock position

$$
\frac{\mathrm{d}}{\mathrm{d} \phi}\left[\left(F(\phi)-\phi F^{\prime}(\phi)\right)\left(\xi-\xi_{\mathrm{A}}\right)\right]=0,
$$

which may be integrated with the initial condition that the shock starts from point B (where $\psi=0$ and $\phi=1$ ) to give the implicit downstream position of the shock as

$$
\xi=\xi_{\mathrm{A}}-\frac{\psi_{\mathrm{R}}}{F(\phi)-\phi F^{\prime}(\phi)} .
$$

This is valid for concentrations in the range $\phi \in\left[\phi_{R}, 1\right]$, with the height of the shock given by (2.17). Shock BC propagates downwards until $\phi=\phi_{\mathrm{R}}$, where it meets the no-mean-flow line $\hat{z}=\hat{z}_{\mathrm{R}}$ at point $\mathrm{C}$ with downstream coordinate

$$
\xi_{\mathrm{C}}=\xi_{\mathrm{A}}-\frac{\psi_{\mathrm{R}}}{F\left(\phi_{\mathrm{R}}\right)} .
$$

This is consistent with (2.16), closing the structure of the breaking wave.

As the asymmetric flux functions are normalised through (2.8) so that their maximum value is the same as that of the quadratic flux, (2.16) and (2.21) imply that the 'lens' has a constant length of $-4 \psi_{\mathrm{R}}$, which is identical to Thornton \& Gray (2008). However, the result of the asymmetry is that both points B and D are shifted to the right as compared to the quadratic flux. This means that the characteristics in the upper and lower portions of the 'lens' are no longer rotationally invariant about the centre of the lens.

\subsection{Non-convex 'lens' structure}

The 'lens' structure for asymmetric flux functions with small amounts of nonconvexity, $0.5<\gamma \leqslant \Gamma$, is similar to the convex 'lens' structure of $\S 2.1$. However, as explained in $\S 1.3$, the non-convexity causes the large particles to display collective motion, with the maximum large particle velocity occurring at concentration $\phi_{\mathrm{M}}$. This causes a slight difference in the upper domain, and an example of the structure is shown in figure $10(b)$ for $\gamma=0.65$. The characteristics of the rarefaction fan ABCA still satisfy (2.17), but for $\phi \in\left[\phi_{\mathrm{R}}, \phi_{\mathrm{M}}\right]$. A semi-shock $\mathrm{AB}$ now separates the rarefaction fan from the small particle region upstream, and is equivalent to the $\phi=\phi_{\mathrm{M}}$ characteristic. Point $\mathrm{B}$ thus has downstream position

$$
\xi_{\mathrm{B}}=\xi_{\mathrm{A}}+\frac{\psi_{\mathrm{R}}}{F^{\prime}\left(\phi_{\mathrm{M}}\right)},
$$

which is shifted even further to the right. Shock BC still satisfies (2.20), but with concentrations in the range $\phi \in\left[\phi_{\mathrm{R}}, \phi_{\mathrm{M}}\right]$. The remainder of the structure is the same as $\S 2.1$ and the length of the 'lens' remains unaffected. 


\section{3. 'Lens-tail' structure}

For larger amounts of asymmetry, $\Gamma<\gamma \leqslant 1$, the greater difference between the maximum speeds of large and small particles and the collective motion of coarse grains combine to produce a new 'lens-tail' structure, shown in figure 10(c) for $\gamma=0.9$. The structure shares some similarities with the structure for normally graded inflow with an asymmetric flux derived by Gajjar \& Gray (2014). A rarefaction fan CDEAC occurs in the lower domain, with characteristics given by (2.9) for $\phi \in\left[0, \phi_{\mathrm{R}}\right]$. However, the upstream region of small particles $\left(\phi^{+}=1\right)$ is separated from the rarefaction fan $\left(\phi^{-}=\phi\right)$ by a shock DE, together with a semi-shock EA that lies adjacent to a non-centred expansion fan EFAE. This non-centred expansion fan forms the lower portion of the 'tail'. Shock DE satisfies (2.15), but with $\phi \in\left[0, \phi_{\mathrm{E}}\right]$ where $\phi_{\mathrm{E}}$ is defined in $(1.10 b)$. Point $\mathrm{E}$ has coordinates $\left(\xi_{\mathrm{E}}, \psi_{\mathrm{E}}\right)$ given by $(2.15)$ with $\phi=\phi_{\mathrm{E}}$

$$
\begin{gathered}
\xi_{\mathrm{E}}=\xi_{\mathrm{C}}+\frac{\psi_{\mathrm{R}}}{F\left(\phi_{\mathrm{E}}\right)+\left(1-\phi_{\mathrm{E}}\right) F^{\prime}\left(\phi_{\mathrm{E}}\right)}=\xi_{\mathrm{C}}+\frac{\psi_{\mathrm{R}} \gamma^{2}}{A_{\gamma}(2 \gamma-1)^{3}}, \\
\psi_{\mathrm{E}}=\psi_{\mathrm{R}}-F^{\prime}\left(\phi_{\mathrm{E}}\right)\left(\xi_{\mathrm{E}}-\xi_{\mathrm{C}}\right)=\psi_{\mathrm{R}} \frac{(1-\gamma)^{2}(3 \gamma-1)}{(2 \gamma-1)^{3}} .
\end{gathered}
$$

Semi-shock EA separates each rarefaction characteristic $\phi^{-}=\phi$ in CDEFC from its image point concentration characteristic $\phi^{+}=\phi^{\circ}$ in EAFE. Using the definition of the image point concentration $\phi^{\circ}(1.9)$, the shock gradient (2.7) and the equation of the rarefaction characteristics (2.9) can be manipulated in a similar manner to (2.9) and (2.11) to give a first-order differential equation for the semi-shock path

$$
\frac{1}{\xi-\xi_{\mathrm{C}}} \frac{\mathrm{d} \xi}{\mathrm{d} \phi}=\frac{F^{\prime \prime}(\phi)}{F^{\prime}\left(\phi^{o}\right)-F^{\prime}(\phi)}=-\frac{8 \gamma}{3 \gamma \phi-(1+\gamma)} .
$$

For the cubic flux, this equation is separable and can be integrated exactly given that the semi-shock starts from point $\mathrm{E}$

$$
\begin{aligned}
\xi_{\mathrm{EA}} & =\xi_{\mathrm{C}}+\frac{\psi_{\mathrm{R}} \gamma^{2}}{A_{\gamma}}\left(\frac{256}{(2 \gamma-1)(3 \gamma \phi-(1+\gamma))^{8}}\right)^{1 / 3}, \\
\psi_{\mathrm{EA}} & =\psi_{\mathrm{R}}-F^{\prime}(\phi)\left(\xi_{\mathrm{EA}}(\phi)-\xi_{\mathrm{C}}\right),
\end{aligned}
$$

with concentration $\phi \in\left[\phi_{\mathrm{E}}, \phi_{\mathrm{R}}\right]$. Point A lies at the end of the semi-shock (2.25) on the no-mean-flow line $\hat{z}=\hat{z}_{\mathrm{R}}$ with $\phi=\phi_{\mathrm{R}}$, and thus has downstream coordinate

$$
\xi_{\mathrm{A}}=\xi_{\mathrm{C}}+\frac{\psi_{\mathrm{R}} \gamma^{2}}{A_{\gamma}}\left(\frac{256}{(2 \gamma-1)\left(\gamma^{2}-\gamma+1\right)^{4}}\right)^{1 / 3} .
$$

Each of the image point concentration $\phi^{o}$ characteristics on the forward side (upstream side as the time-like direction is to the left) of the semi-shock EA lies locally tangential and forms a non-centred expansion fan in EAFE. Each characteristic has equation

$$
\psi-\psi_{\mathrm{EA}}(\phi)=-F^{\prime}\left(\phi^{o}\right)\left(\xi-\xi_{\mathrm{EA}}(\phi)\right),
$$




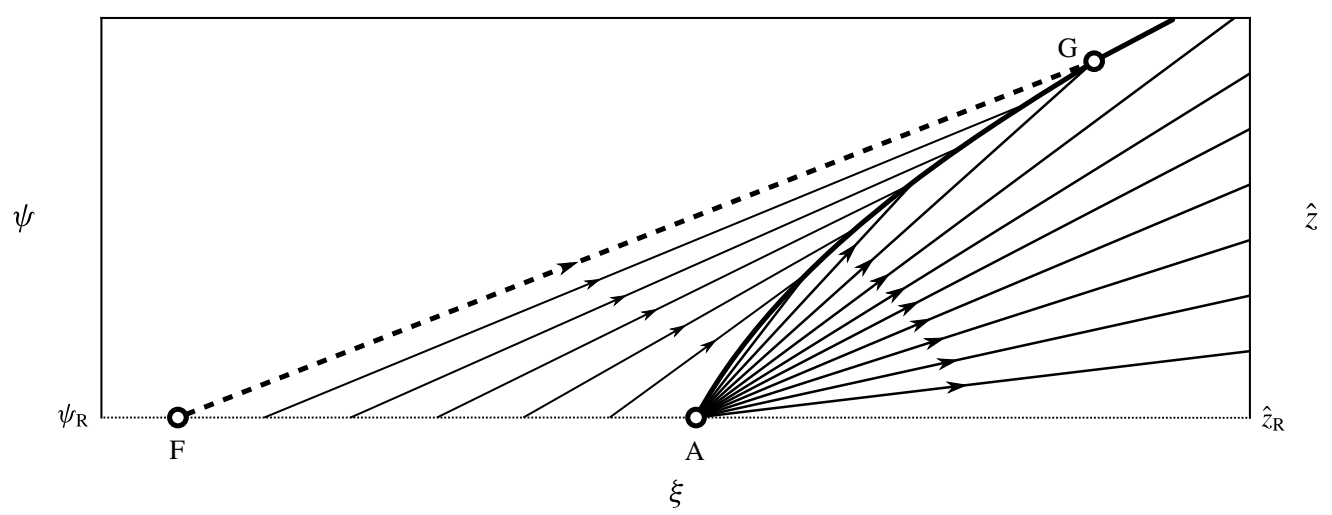

FIGURE 11. A sketch of the upper part of the 'lens-tail' structure, where compression wave FAGF interacts with the rarefaction fan centred at $A$ to form shock AG. The concentration change along either side of the shock is governed by (2.32), whilst the shock position is given by (2.31). Note that the diagram is not to scale and that FG is not tangential at $\mathrm{G}$.

upon which the concentration has a constant value of $\phi^{o}$ with $\phi \in\left[\phi_{\mathrm{E}}, \phi_{\mathrm{R}}\right]$. The characteristics each meet the no-mean-flow line at $\xi_{\mathrm{FA}}(\phi)$, which is given by equating (2.25) and (2.27) with $\psi=\psi_{\mathrm{R}}$

$$
\xi_{\mathrm{FA}}=\xi_{\mathrm{C}}-\frac{\psi_{\mathrm{R}} \gamma}{F^{\prime}\left(\phi^{o}\right)}\left(\frac{4}{(2 \gamma-1)(3 \gamma \phi-(1+\gamma))^{2}}\right)^{1 / 3}, \quad \phi \in\left[\phi_{\mathrm{E}}, \phi_{\mathrm{R}}\right] .
$$

Point $\mathrm{F}$ is the furthest upstream part of the breaking wave and is given by the $\phi_{\mathrm{E}}^{o}$ characteristic that is tangential at point $\mathrm{E}$,

$$
\xi_{\mathrm{F}}=\xi_{\mathrm{C}}+\frac{\psi_{\mathrm{R}} \gamma}{A_{\gamma}(2 \gamma-1)(1-\gamma)} .
$$

The solution in the upper domain $\left(\hat{z}>\hat{z}_{\mathrm{R}}\right)$ matches the lower domain $\left(\hat{z}<\hat{z}_{\mathrm{R}}\right)$ along the no-mean-flow line $\psi=\psi_{\mathrm{R}}$. As $F^{\prime}\left(\phi_{\mathrm{R}}\right)=0$, the $\phi_{\mathrm{R}}$ characteristic lies horizontally between points $\mathrm{C}$ and $\mathrm{A}$ and gives concentration $\phi=\phi_{\mathrm{R}}$, whilst (2.28) governs the concentration between A and F. A characteristic of concentration $\phi^{o}$ emanates into the upper region from each point between $\mathrm{F}$ and $\mathrm{A}$

$$
\psi=\psi_{\mathrm{R}}-F^{\prime}\left(\phi^{o}\right)\left(\xi-\xi_{\mathrm{FA}}(\phi)\right),
$$

with $\phi \in\left[\phi_{\mathrm{E}}, \phi_{\mathrm{R}}\right]$ implying that $\phi_{\mathrm{R}}^{o} \leqslant \phi^{o} \leqslant 1$. The $\phi_{\mathrm{R}}^{o}$ characteristic originates from A, whilst the $\phi=1$ characteristic originates from F. All the characteristics form a compression wave FGAF (Whitham 1974; Rhee et al. 1986); each characteristic has a steeper gradient than the characteristic immediately to its left, as shown in figure 11. This is the upper portion of the 'tail' region. The 'lens' region is formed from an expansion fan AGBCA centred at A whose characteristics are given by (2.17) with $\phi_{\mathrm{R}} \leqslant \phi \leqslant \phi_{\mathrm{R}}^{o o}$. These rarefaction characteristics collide with the compression wave characteristics (2.30) to form a shock AG. A full derivation of the governing equations 
for the shock is provided in appendix B. The shock coordinates are given implicitly given by

$$
\begin{aligned}
\xi_{\mathrm{AG}}\left(\phi_{\text {left }}, \phi_{\text {right }}\right) & =\frac{F^{\prime}\left(\phi_{\text {left }}\right) \tilde{\xi}_{\mathrm{FA}}\left(\phi_{\text {left }}\right)-F^{\prime}\left(\phi_{\text {right }}\right) \xi_{\mathrm{F}}}{F^{\prime}\left(\phi_{\text {left }}\right)-F^{\prime}\left(\phi_{\text {right }}\right)}, \\
\psi_{\mathrm{AG}}\left(\phi_{\text {left }}, \phi_{\text {right }}\right) & =\psi_{\mathrm{R}}-\frac{F^{\prime}\left(\phi_{\text {left }}\right) F^{\prime}\left(\phi_{\text {right }}\right)}{F^{\prime}\left(\phi_{\text {left }}\right)-F^{\prime}\left(\phi_{\text {right }}\right)}\left(\tilde{\xi}_{\mathrm{FA}}\left(\phi_{\text {left }}\right)-\xi_{\mathrm{F}}\right),
\end{aligned}
$$

where $\xi_{\mathrm{FA}}(\phi)=\tilde{\xi}_{\mathrm{FA}}\left(\phi^{\circ}\right)$, and concentrations $\phi_{\text {left }}$ and $\phi_{\text {right }}$ on the left (upstream) and right (downstream) sides of the shock are related by

$$
\begin{aligned}
\frac{\mathrm{d} \phi_{\text {right }}}{\mathrm{d} \phi_{\text {left }}}= & \frac{F\left(\phi_{\text {left }}\right)-F\left(\phi_{\text {right }}\right)-\left(\phi_{\text {left }}-\phi_{\text {right }}\right) F^{\prime}\left(\phi_{\text {right }}\right)}{F\left(\phi_{\text {left }}\right)-F\left(\phi_{\text {right }}\right)-\left(\phi_{\text {left }}-\phi_{\text {right }}\right) F^{\prime}\left(\phi_{\text {left }}\right)} \\
& \times\left[\frac{F^{\prime}\left(\phi_{\text {right }}\right) F^{\prime \prime}\left(\phi_{\text {left }}\right)}{F^{\prime}\left(\phi_{\text {left }}\right) F^{\prime \prime}\left(\phi_{\text {right }}\right)}-\frac{F^{\prime}\left(\phi_{\text {left }}\right)-F^{\prime}\left(\phi_{\text {right }}\right)}{F^{\prime \prime}\left(\phi_{\text {right }}\right)} \frac{\tilde{\xi}_{\mathrm{FA}}^{\prime}\left(\phi_{\text {left }}\right)}{\tilde{\xi}_{\mathrm{FA}}\left(\phi_{\text {left }}\right)-\xi_{\mathrm{F}}}\right] .
\end{aligned}
$$

The shock entropy condition (Oleinik 1959; Rhee et al. 1986; Gajjar \& Gray 2014) requires that $\mathrm{AG}$ must initially start tangential to the rarefaction fan, and as $\phi_{\text {left }}=\phi_{\mathrm{R}}^{o}$ at point A, (1.9) implies that $\phi_{\text {right }}=\left(\phi_{\mathrm{R}}^{o}\right)^{o}=\phi_{\mathrm{R}}^{o o}$ at this point. The relationship between $\phi_{\mathrm{R}}, \phi_{\mathrm{R}}^{o}$ and $\phi_{\mathrm{R}}^{o o}$ is illustrated in figure 8. Equation (2.32) may be numerically integrated from $\phi_{\text {left }}=\phi_{\mathrm{R}}^{o}$ to $\phi_{\text {left }}=1$ to give $\phi_{\text {right }}=\phi_{\mathrm{G}}$ at point $\mathrm{G}$ and the coordinates $\left(\xi_{\mathrm{G}}, \psi_{\mathrm{G}}\right)$ of point $\mathrm{G}$ are given by (2.31). Shock GB separates the upstream region of pure small particles $\left(\phi^{-}=1\right)$ from the rarefaction fan in the 'lens' region $\left(\phi^{+}=\phi\right)$. Combining (2.7) and (2.17) using the chain rule shows that GB satisfies

$$
\frac{\mathrm{d}}{\mathrm{d} \phi}\left[\left(F(\phi)+(1-\phi) F^{\prime}(\phi)\right)\left(\xi-\xi_{\mathrm{A}}\right)\right]=0 .
$$

The shock starts from point $\mathrm{G}$, and hence (2.33) can be integrated to give

$$
\begin{aligned}
\xi_{\mathrm{GB}} & =\xi_{\mathrm{A}}+\frac{F\left(\phi_{\mathrm{G}}\right)+\left(1-\phi_{\mathrm{G}}\right) F^{\prime}\left(\phi_{\mathrm{G}}\right)}{F(\phi)+(1-\phi) F^{\prime}(\phi)}\left(\xi_{\mathrm{G}}-\xi_{\mathrm{A}}\right), \\
\psi_{\mathrm{GB}} & =\psi_{\mathrm{R}}-F^{\prime}(\phi)\left(\xi_{\mathrm{GB}}(\phi)-\xi_{\mathrm{A}}\right),
\end{aligned}
$$

with $\phi \in\left[\phi_{\mathrm{B}}, \phi_{\mathrm{G}}\right]$. A final shock BC satisfying (2.19) separates the downstream region of large particles $\left(\phi^{+}=0\right)$ from the rarefaction fan $\left(\phi^{-}=\phi\right)$. Shock BC must meet the no-mean-flow line at $\mathrm{C}$, where $\phi=\phi_{\mathrm{R}}$, and since $\xi_{\mathrm{C}}-\xi_{\mathrm{A}}$ is given by (2.26), equation (2.19) can be integrated to give

$$
\begin{aligned}
\xi_{\mathrm{BC}} & =\xi_{\mathrm{A}}-\frac{\mathscr{G}}{F(\phi)-\phi F^{\prime}(\phi)}=\xi_{\mathrm{A}}-\frac{\mathscr{G}}{A_{\gamma} \phi^{2}(1+\gamma-2 \gamma \phi)}, \\
\psi_{\mathrm{BC}} & =\psi_{\mathrm{R}}-\frac{F^{\prime}(\phi) \mathscr{G}}{F(\phi)-\phi F^{\prime}(\phi)},
\end{aligned}
$$


with $\phi \in\left[\phi_{\mathrm{R}}, \phi_{\mathrm{B}}\right]$ and

$$
\mathscr{G}=\left(\xi_{\mathrm{C}}-\xi_{\mathrm{A}}\right) F\left(\phi_{\mathrm{R}}\right)=\frac{\xi_{\mathrm{C}}-\xi_{\mathrm{A}}}{4}=\frac{\psi_{\mathrm{R}} \gamma^{2}}{A_{\gamma}}\left(\frac{4}{(2 \gamma-1)\left(\gamma^{2}-\gamma+1\right)^{4}}\right)^{1 / 3} .
$$

The shock reaches the top of the 'lens' at point B where $\psi=0$, and hence (2.35) determines both $\phi_{\mathrm{B}}$ and $\xi_{\mathrm{B}}$.

A requirement for the 'lens-tail' solution to form is that point $\mathrm{E}$ must reside in the lower domain, which occurs when $\phi_{E}<\phi_{R}$. Instead, if $\phi_{E}>\phi_{R}$, then point $E$ would lie in the upper domain and shock DE would continue up to the no-mean-flow line, forming the non-convex 'lens' structure of $\$ 2.2$. The transition between the 'lens' solution and the 'lens-tail' solution thus occurs when point $\mathrm{E}$ lies on the no-mean-flow line $\psi=\psi_{\mathrm{R}}$, i.e. when $\phi_{\mathrm{R}}=\phi_{\mathrm{E}}$. The definition $F^{\prime}\left(\phi_{\mathrm{R}}\right)=0$ implies that for the cubic flux (1.5)

$$
\phi_{\mathrm{R}}=\frac{1+\gamma \pm \sqrt{\gamma^{2}-\gamma+1}}{3 \gamma},
$$

and equating this with $\phi_{\mathrm{E}}(1.10 b)$ gives the quadratic equation

$$
5 \gamma^{2}-5 \gamma+1=0
$$

As the cubic flux (1.5) is non-convex when $\gamma>0.5$, the transition between the nonconvex 'lens' and 'lens-tail' solutions takes place at the larger of the two roots of (2.38), namely

$$
\gamma=\Gamma=(5+\sqrt{5}) / 10
$$

\subsection{Solution in physical coordinates}

Following Thornton \& Gray (2008), transformations (2.1) and (2.4) from ( $x, z)$ to $(\xi, \psi)$ coordinates mean that the steady-state structures shown in figure 10 describe all of the breaking size-segregation waves that develop under steady uniform flow. They are valid for waves that exist between all vertical heights $H_{\text {down }}$ and $H_{u p}$, for any constant segregation number $S_{r}$ and for any monotonically increasing velocity profile $u(z)$. For example, consider the simple linear velocity profile,

$$
u=\alpha+2(1-\alpha) z, \quad 0 \leqslant \alpha<1
$$

where $\alpha$ is the parameter that controls the amount of shear across the layer. The case of $\alpha=0$ represents simple shear with zero basal velocity, whilst $\alpha=1$ corresponds to plug flow. Not only is this the simplest non-trivial velocity field that highlights all the major features of the breaking-wave structure, but it is also a good leading-order approximation to the velocity field measured in the (shallow) moving-bed flume experiments of $\S 1.1$. From (1.13), the breaking wave travels downstream with velocity

$$
u_{\text {wave }}=\alpha+(1-\alpha)\left(H_{u p}+H_{\text {down }}\right),
$$

and so the relative downstream velocity $\hat{u}$ becomes

$$
\hat{u}=(1-\alpha)\left(H_{u p}-H_{\text {down }}\right)(2 \hat{z}-1) .
$$


The no-mean-flow line $\hat{z}_{\mathrm{R}}=1 / 2$ lies halfway between the vertical heights $H_{\text {down }}$ and $H_{u p}$ in untransformed variables. Transformation (2.4) means that $z$ and $\psi$ have a quadratic relation,

$$
\psi=(1-\alpha)\left(H_{u p}-H_{\text {down }}\right)\left(\hat{z}^{2}-\hat{z}\right),
$$

with the transformed no-mean-flow line $\psi_{\mathrm{R}}=-(1-\alpha)\left(H_{u p}-H_{\text {down }}\right) / 4$. Inverting equation (2.43) gives $\hat{z}$ as a function of $\psi$, with the positive and negative roots for the upper and lower regions, respectively. Figure 12 shows the steady breaking waves in physical coordinates for linear shear with $\alpha=0.5, S_{r}=1, H_{u p}=0.9$ and $H_{\text {down }}=0.1$ for $\gamma=0.35,0.65$ and 0.9 in $(a),(b)$ and $(c)$ respectively.

\subsection{Comparison with solution for the quadratic flux}

The asymmetric cubic flux function (1.5) leads to a number of differences in the structures shown in figure 12 and that of the quadratic flux function (1.2) shown in figure $9(e)$. The two 'lens'-like structures in figure $12(a, b)$ have a strong similarity to the symmetric 'lens' structure. As the normalisation of the flux function (2.8) implies that (2.21) is independent of $\gamma$, the 'lens' length is identical to the quadratic 'lens' length $(1-\alpha)\left(H_{u p}-H_{d o w n}\right)^{2} / S_{r}$. However, with an asymmetric flux, the 'lens' structures have no rotational symmetry. The asymmetry also means the maximum rise velocity of large particles is less than the maximum percolation velocity of fines, causing point $\mathrm{B}$ to lie further downstream. When $\Gamma<\gamma \leqslant 1$, a few large particles surrounded by many fines rise very slowly, and so are swept a long way downstream before recirculating. This causes the additional 'tail'-like region, and substantially increases the length of the breaking wave. The colour map shows how the concentration in the 'tail' is very similar to $\phi=1$ of the surrounding region of pure small particles, reflecting the very small number of large particles that circulate slowly through this region. Most of the large particles still rise at a moderate speed through the 'lens' region; however, the small particles percolate down very quickly. This leaves a higher concentration of coarse grains in the middle of the 'lens', shown by a stronger green hue. Interestingly, the length of this 'lens' region in the 'lens-tail' structure remains very close to the length of the 'lens' structures. Comparing (2.26) with (2.16) shows that the length of the 'lens' within the 'lens-tail' structure is, at most, only $9 \%$ less than the length of the 'lens' structure.

\section{Recirculating particle motion through the breaking wave}

The recirculating motion of the grains can be understood by examining the particle paths as they pass through the breaking wave. Following Thornton et al. (2006) and Thornton \& Gray (2008), the particle paths of the large (superscript $l$ ) and small grains (superscript $s$ ) are given by

$$
\frac{\mathrm{d} x^{v}}{\mathrm{~d} t}=u^{v}, \quad \frac{\mathrm{d} z^{v}}{\mathrm{~d} t}=w^{v}, \quad(v=l, s) .
$$

The normal constituent velocities $w^{v}$ are given by (1.3) and the downstream constituent velocities are assumed to be equal to the downstream bulk velocity (1.11), $u^{s}=u^{l}=u$. Eliminating the time dependence from (3.1) and employing the non-dimensionalisation (2.1) gives the non-dimensional particle paths

$$
\hat{u}(\hat{z}) \frac{\mathrm{d} \hat{z}^{v}}{\mathrm{~d} \hat{x}^{v}}=\hat{w}^{v}(\phi), \quad(v=l, s),
$$



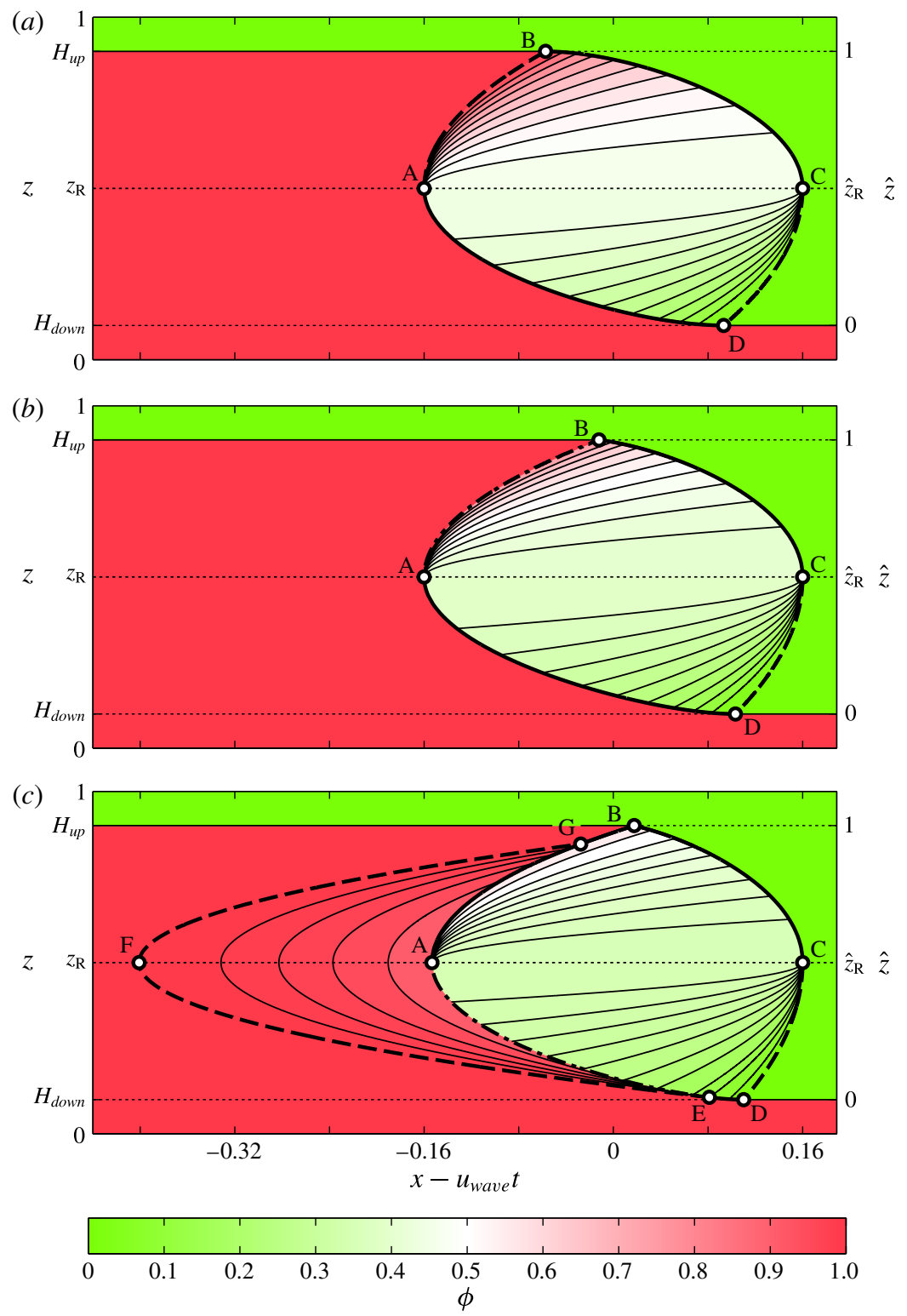

FIGURE 12. The breaking wave that develops between $H_{u p}=0.9$ and $H_{\text {down }}=0.1$ is shown in physical coordinates $(x, z)$ in a frame translating with velocity $u_{\text {wave }}(1.13)$. The bulk velocity $u(z)$ follows a linear shear profile (2.40) with $\alpha=0.5$. The three different structures that arise for the asymmetric cubic flux (1.5) with $S_{r}=1$ are shown in $(a-c)$ for $\gamma=0.35,0.65$ and 0.9 , respectively. The asymmetry in the large and small particle velocities that result from the asymmetric flux function causes point B to be swept further downstream in the two 'lens'-like structures $(a)$ and $(b)$ compared to the symmetric quadratic flux shown in figure $9(e)$. These asymmetric velocities are even more significant in $(c)$, where the slow rise rate of large particles surrounded by many fines means that some large particles are swept a long way upstream before recirculating. This results in the 'tail' region EFGAE. The concentration map reflects how only a small number of large particles recirculate through this region. Most large particles still rise at a moderate velocity, and recirculate in the 'lens' region. 
which simplify further in velocity-averaged coordinates (2.4) to give

$$
\frac{\mathrm{d} \psi^{l}}{\mathrm{~d} \xi^{l}}=\frac{F(\phi)}{1-\phi}, \quad \frac{\mathrm{d} \psi^{s}}{\mathrm{~d} \xi^{s}}=-\frac{F(\phi)}{\phi} .
$$

The concentration fields derived in $\S 2$ can be used with (3.3) to calculate the particle paths through each of the breaking-wave structures.

\subsection{Recirculation through the 'lens' structures}

First consider the recirculation through a breaking wave with a convex 'lens' structure when $\gamma \leqslant 0.5$. Suppose a small particle starts at a height $\hat{z}_{\text {enter }}^{s}>\hat{z}_{\mathrm{R}}$, equivalent to transformed height $\psi_{\text {enter }}^{s}$. The small grains are moving faster than the breaking wave in the upper region, and so they are swept downstream to the right before crossing $\mathrm{AB}$ and entering the 'lens' at downstream distance

$$
\xi_{\text {enter }}^{s}=\xi_{\mathrm{A}}+\frac{\psi_{\text {enter }}^{s}-\psi_{\mathrm{R}}}{F^{\prime}(1)}
$$

Although the local small particle velocity is given by $(3.3 b)$, the local concentration changes through the rarefaction fan ABCA according to characteristics (2.17). These characteristics may be differentiated with respect to $\phi$ as in (2.13), whilst the chain rule may be used to write the small particle velocity $(3.3 b)$ in a similar manner to (2.12). Combining these equations shows that the small particle motion through the upper part of the 'lens' is governed by ODE (2.19), with $\xi^{s}$ instead of $\xi$. As the small particle enters the 'lens' at $\xi_{\text {enter }}^{s}$ (3.4) when $\phi=1$, the motion through ABCA is given by

$$
\xi^{s}=\xi_{\mathrm{A}}+\frac{\psi_{\text {enter }}^{s}-\psi_{\mathrm{R}}}{F(\phi)-\phi F^{\prime}(\phi)},
$$

with $\phi \in\left[\phi_{\mathrm{R}}, 1\right]$. The small particle continues along this path until it crosses the nomean-flow line $\mathrm{AC}$ at

$$
\xi_{\mathrm{AC}}^{s}=\xi_{\mathrm{A}}+\frac{\psi_{\text {enter }}^{s}-\psi_{\mathrm{R}}}{F\left(\phi_{\mathrm{R}}\right)}=\xi_{\mathrm{A}}+4\left(\psi_{\text {enter }}^{s}-\psi_{\mathrm{R}}\right),
$$

with the last equation a result of the normalisation (2.8). The motion through the lower region CDAC is similarly governed by velocity (3.3b) and characteristics (2.9), which combine to give the differential equation

$$
\frac{\mathrm{d}}{\mathrm{d} \phi}\left[\left(F(\phi)-\phi F^{\prime}(\phi)\right)\left(\xi^{s}-\xi_{\mathrm{C}}\right)\right]=0 .
$$

Since the small particle crosses the no-mean-flow line at $\xi^{s}=\xi_{\mathrm{AC}}^{s}$ when $\phi=\phi_{\mathrm{R}}$, equations (2.21), (3.6) and (3.7) imply that the motion through the lower region $\mathrm{CDAC}$ is given by

$$
\xi^{s}=\xi_{\mathrm{C}}+\frac{\psi_{\text {enter }}^{s}}{F(\phi)-\phi F^{\prime}(\phi)},
$$

with $\phi \in\left[\phi_{\mathrm{DA}}^{s}, \phi_{\mathrm{R}}\right]$. The small particle exits the breaking wave across DA, with equations (2.9), (2.15) and (3.8) giving both the concentration $\phi_{\mathrm{DA}}^{s}$ and the exit height $\psi_{\mathrm{DA}}^{s}$. 
Similarly, consider a large particle that starts in the lower region at a height $\hat{z}^{l}=\hat{z}_{\text {enter }}^{l}<\hat{z}_{\mathrm{R}}$, corresponding to $\psi^{l}=\psi_{\text {enter }}^{l}$. The large particles initially move slower than the breaking wave, and so are swept upstream to the left until they meet CD at distance

$$
\xi_{\text {enter }}^{l}=\xi_{\mathrm{C}}+\frac{\psi_{\mathrm{R}}-\psi_{\text {enter }}^{l}}{F^{\prime}(0)}
$$

The concentration within the lower part of the 'lens' CDAC is governed by characteristics (2.9) whilst the local velocity is given by (3.3a); these combine to give differential equation (2.14) with $\xi^{l}$ replacing $\xi$. With the initial condition $\phi=0$ at $\xi^{l}=\xi_{\text {enter }}^{l}$, the large particle path through CDAC is given by

$$
\xi^{l}=\xi_{\mathrm{C}}+\frac{\psi_{\mathrm{R}}-\psi_{\text {enter }}^{l}}{F(\phi)+(1-\phi) F^{\prime}(\phi)}
$$

Each large particle crosses the no-mean-flow line $\mathrm{AC}$ at distance

$$
\xi_{\mathrm{AC}}^{l}=\xi_{\mathrm{C}}+\frac{\psi_{\mathrm{R}}-\psi_{\text {enter }}^{l}}{F\left(\phi_{\mathrm{R}}\right)}=\xi_{\mathrm{C}}+4\left(\psi_{\mathrm{R}}-\psi_{\text {enter }}^{l}\right) .
$$

In the upper part of the 'lens', characteristics (2.17) govern the concentration at a point $(\xi, \psi)$, and can be combined with $(3.3 a)$ to give a governing differential equation that resembles (2.14)

$$
\frac{\mathrm{d}}{\mathrm{d} \phi}\left[\left(F(\phi)+(1-\phi) F^{\prime}(\phi)\right)\left(\xi^{l}-\xi_{\mathrm{A}}\right)\right]=0 .
$$

The initial condition that $\phi=\phi_{\mathrm{R}}$ at $\xi^{l}=\xi_{\mathrm{AC}}^{l}$, along with (2.21) and (3.11) give the large particle path through ABCA as

$$
\xi^{l}=\xi_{\mathrm{A}}-\frac{\psi_{\text {enter }}^{l}}{F(\phi)+(1-\phi) F^{\prime}(\phi)},
$$

where $\phi \in\left[\phi_{\mathrm{R}}, \phi_{\mathrm{BC}}^{l}\right]$. The large particle exits the breaking wave by crossing shock $\mathrm{BC}$ when $\phi=\phi_{\mathrm{BC}}^{l}$ and $\xi^{l}=\xi_{\mathrm{BC}}^{l}$. These are found by equating (2.20) and (3.13), with the exit height $\psi^{l}=\psi_{\mathrm{BC}}^{l}$ given by (2.17). After exiting the breaking wave, the large particles continue to move downstream at a constant height $\psi_{\mathrm{BC}}^{l}$.

The particle paths through the non-convex 'lens' structure that forms for $0.5<\gamma \leqslant$ $\Gamma$ are identical to the above, except that the small particles enter the breaking wave by crossing semi-shock AC (2.17) when $\phi=\phi_{\mathrm{M}}(1.10 a)$. Thus, the distance $\xi_{\text {enter }}^{s}$ at which they first enter the 'lens' is given by

$$
\xi_{\text {enter }}^{s}=\xi_{\mathrm{A}}-\frac{\psi_{\text {enter }}^{s}-\psi_{\mathrm{R}}}{F^{\prime}\left(\phi_{\mathrm{M}}\right)}
$$

whilst equation (3.5) governing the motion of the small particles through the upper region is valid for $\phi \in\left[\phi_{\mathrm{R}}, \phi_{\mathrm{M}}\right]$.

The small particle paths are parameterised by $\psi_{\text {enter }}^{s}$, and are given implicitly by (3.4) or (3.14) (for $\gamma \leqslant 0.5$ and $0.5<\gamma \leqslant \Gamma$ respectively), (3.5), (3.6) and (3.8), whilst the large particle paths are parameterised by $\psi_{\text {enter }}^{l}$ and are given by (3.9)-(3.13). The paths can be transformed back from velocity averaged $(\xi, \psi)$ variables to physical $(x, z)$ variables using the results of $\S 2.4$, and are shown in figure $13(a, b)$ for $\gamma=0.35$ and $\gamma=0.65$, respectively. 

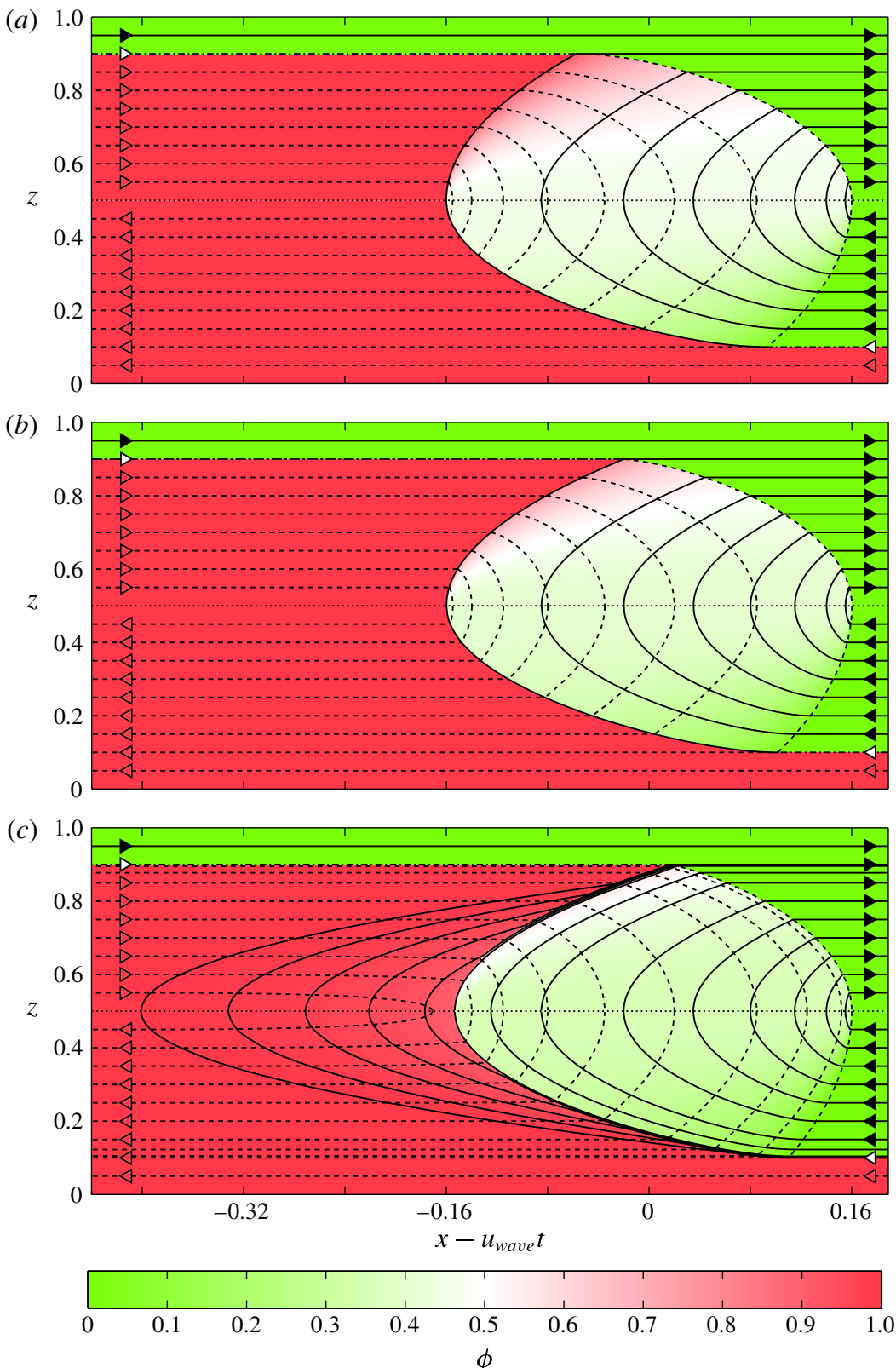

FIGURE 13. The particle paths within the breaking wave are shown superimposed on top of the concentration field for each of the cases in figure 12. The large particles are shown using a solid line with a black arrow, whilst the small particles are shown using a dashed line with a red arrow. The dash-dot line with white arrows shows the upstream and downstream shocks where large particles propagate along the upper side and small particles propagate along the lower side. The boundary of the breaking wave, where particles recirculate between the vertical heights $H_{u p}$ and $H_{\text {down }}$, is defined by the highest small particle path and lowest large particle path. 

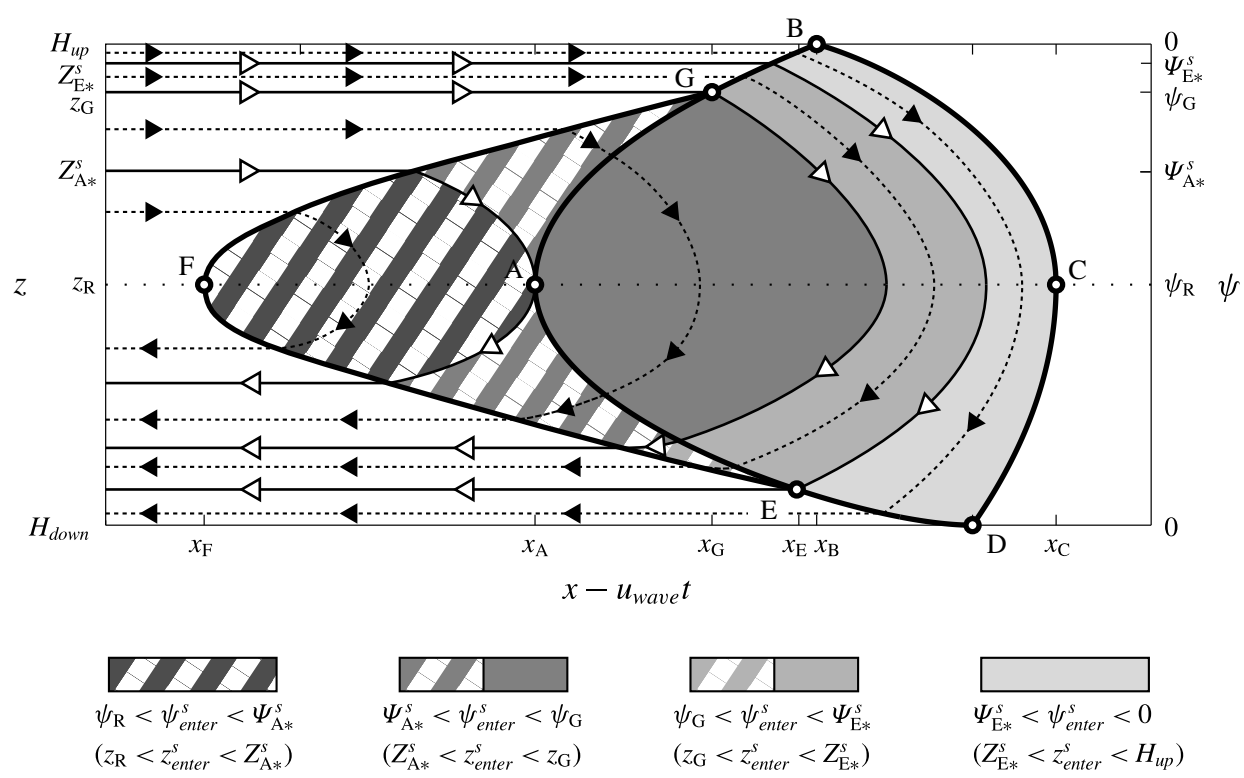

FIGURE 14. A sketch showing how the small particles may pass through different parts of the 'lens-tail' structure depending on their initial starting height (not to scale). There are two critical heights $\Psi_{\mathrm{A} *}^{s}$ and $\Psi_{\mathrm{E} *}^{s}$, corresponding to physical heights $Z_{\mathrm{A} *}^{s}=z\left(\Psi_{\mathrm{A} *}^{s}\right)$ and $Z_{\mathrm{E} *}^{s}=z\left(\Psi_{\mathrm{E} *}^{s}\right)$, which define small particle paths that pass through points $\mathrm{A}$ and $\mathrm{E}$, respectively. These paths, along with the path passing through point $\mathrm{G}$, are shown with thin solid lines and white arrows. The small particles starting at an initial height $\Psi_{\mathrm{E} *}^{s} \leqslant$ $\psi_{\text {enter }}^{s}<0$ (physical height $Z_{\mathrm{E} *}^{s} \leqslant z_{\text {enter }}^{s}<H_{u p}$ ) just pass through the 'lens', whilst those starting at an initial height $\psi_{\mathrm{G}} \leqslant \psi_{\text {enter }}^{s}<\Psi_{\mathrm{E} *}^{s}$ (physical height $z_{\mathrm{G}} \leqslant z_{\text {enter }}^{s}<Z_{\mathrm{E} *}^{s}$ ) pass through the 'lens' and the lower portion of the 'tail'. Small grains starting at $\Psi_{\mathrm{A} *}^{s} \leqslant$ $\psi_{\text {enter }}^{s}<\psi_{\mathrm{G}}$ (physical height $Z_{\mathrm{A} *}^{s} \leqslant z_{\text {enter }}^{s}<z_{\mathrm{G}}$ ) pass through the upper portion of the 'tail', the 'lens' and the lower portion of the 'tail'. Finally, small grains starting closest to the no-mean-flow line $\psi_{\mathrm{R}}<\psi_{\text {enter }}^{s}<\Psi_{\mathrm{A} *}^{s}$ (at physical heights $z_{\mathrm{R}}<z_{\text {enter }}^{s}<Z_{\mathrm{A} *}^{s}$ ) only recirculate through the 'tail' region. The 'lens' and 'tail' regions are shown with solid colour and cross-shading, respectively, whilst sample particle paths starting at each of these heights are shown using thin dashed lines with black arrows. The thick solid lines mark the boundaries of the breaking wave.

\subsection{Recirculation through the 'lens-tail' structure}

Just like the recirculation within the 'lens' structure, the small particle (superscript $s$ ) motion is parameterised by $\psi_{\text {enter }}^{s}$. As summarised in figure 14, the initial starting height $\psi_{\text {enter }}^{s}$ determines whether the small grains may pass through only the 'lens', both the 'lens' and 'tail' regions, or just through the 'tail'. The small particles starting at a height $\Psi_{\mathrm{E} *}^{s} \leqslant \psi_{\text {enter }}^{s}<0$ would cross shock GB at downstream distance $\xi_{\mathrm{GB}}^{s}$, recirculate through the 'lens' and exit the breaking wave across DE. Solving $\psi_{\mathrm{GB}}=\psi_{\text {enter }}^{s}$ in (2.34) gives the position $\xi_{\mathrm{GB}}^{s}$ at which the particle enters the breaking wave, and the concentration $\phi_{\mathrm{GB}}^{s}$ on the downstream side of shock GB at this point. The motion through the upper portion of the 'lens' AGBCA is governed by (2.19), with $\xi^{s}$ replacing $\xi$. This can be integrated subject to $\xi^{s}=\xi_{\mathrm{GB}}^{s}$ at $\phi=\phi_{\mathrm{GB}}^{s}$ to give

$$
\xi^{s}=\xi_{\mathrm{A}}+\frac{F\left(\phi_{\mathrm{GB}}^{s}\right)-\phi_{\mathrm{GB}}^{s} F^{\prime}\left(\phi_{\mathrm{GB}}^{s}\right)}{F(\phi)-\phi F^{\prime}(\phi)}\left(\xi_{\mathrm{GB}}^{s}-\xi_{\mathrm{A}}\right),
$$


where $\phi \in\left[\phi_{\mathrm{R}}, \phi_{\mathrm{GB}}^{s}\right]$ and height $\psi^{s}$ is given by (2.17). The particles cross the no-meanflow line $\mathrm{AC}$ at a distance $\xi_{\mathrm{AC}}^{s}$, given by $\phi=\phi_{\mathrm{R}}$ in (3.15). This provides the initial condition for (3.7), governing the motion through the lower part of the 'lens' CDEFC, which integrates to give

$$
\xi^{s}=\xi_{\mathrm{C}}+\frac{\frac{1}{4}\left(\xi_{\mathrm{AC}}^{s}-\xi_{\mathrm{C}}\right)}{F(\phi)-\phi F^{\prime}(\phi)},
$$

with the concentration $\phi$ in the range $\phi \in\left[\phi_{\mathrm{DE}}^{s}, \phi_{\mathrm{R}}\right]$. The particles exit the breaking wave across shock DE at downstream distance $\xi_{\mathrm{DE}}^{s}$, which is found by equating (3.16) with (2.15). This also gives the concentration $\phi_{\mathrm{DE}}^{s}$, with the exit height $\psi_{\mathrm{DE}}^{s}$ given by (2.9).

As shown in figure 14, there is a critical initial height $\Psi_{\mathrm{E} *}^{s}$ from which a small particle passes through point E. It is calculated by equating $\xi^{s}=\xi_{\mathrm{E}}$ in (3.16) and substituting back into (3.15) and (2.17). Small grains starting below $\Psi_{\mathrm{E*}}^{s}$ at an initial height $\psi_{\mathrm{G}} \leqslant \psi_{\text {enter }}^{s}<\Psi_{\mathrm{E} *}^{s}$ enter the 'lens' across GB, travel through the 'lens' according to (2.19) and (3.16), before passing through the lower part of the 'tail' and exiting the breaking wave across EF. The small particle crosses from the 'lens' to the 'tail' at $\left(\xi_{\mathrm{EA}}^{s}, \psi_{\mathrm{EA}}^{s}\right)$ when $\phi=\phi_{\mathrm{EA}}^{s}$, which are given by equating (3.16) with (2.25). The lower portion of the 'tail' EAFE is spanned by characteristics (2.27) of concentration $\phi^{\circ}$; following Gajjar \& Gray (2014), these may be implicitly differentiated with respect to $\phi^{o}$

$$
\frac{\mathrm{d} \psi}{\mathrm{d} \phi^{o}}-\frac{\mathrm{d} \psi_{\mathrm{EA}}}{\mathrm{d} \phi^{o}}=-F^{\prime \prime}\left(\phi^{o}\right) \xi-F^{\prime}\left(\phi^{o}\right) \frac{\mathrm{d} \xi}{\mathrm{d} \phi^{o}}+\frac{\mathrm{d}}{\mathrm{d} \phi^{o}}\left(F^{\prime}\left(\phi^{o}\right) \xi_{\mathrm{EA}}(\phi)\right),
$$

and combined with $(3.3 b)$ using the chain rule to give the inhomogeneous first-order differential equation

$$
\frac{\mathrm{d}}{\mathrm{d} \phi^{o}}\left(\left(F\left(\phi^{o}\right)-\phi^{o} F^{\prime}\left(\phi^{o}\right)\right) \xi^{s}\right)=-\phi^{o} \frac{\mathrm{d}}{\mathrm{d} \phi^{o}}\left(\psi_{\mathrm{EA}}+F^{\prime}\left(\phi^{o}\right) \xi_{\mathrm{EA}}(\phi)\right) .
$$

Integrating by parts with the initial condition $\phi=\phi_{\mathrm{EA}}^{s}$ at $\xi^{s}=\xi_{\mathrm{EA}}^{s}$ gives

$$
\begin{aligned}
\xi^{s} & =\xi_{\mathrm{C}}+\frac{g_{1}\left(\phi_{\mathrm{EA}}^{s}\right)\left(\xi_{\mathrm{EA}}^{s}-\xi_{\mathrm{C}}\right)+g_{2}(\phi)-g_{2}\left(\phi_{\mathrm{EA}}^{s}\right)}{g_{1}(\phi)}, \\
\psi^{s} & =\psi_{\mathrm{EA}}(\phi)-F^{\prime}\left(\phi^{o}\right)\left(\xi^{s}(\phi)-\xi_{\mathrm{EA}}(\phi)\right),
\end{aligned}
$$

where $\phi \in\left[\phi_{\mathrm{E}}, \phi_{\mathrm{EA}}^{s}\right]$ and the functions $g_{1}(u), g_{2}(u)$ are defined as

$$
\begin{aligned}
& g_{1}(u)=\frac{A_{\gamma}}{4 \gamma} u(1+\gamma-\gamma u)^{2}, \\
& g_{2}(u)=\psi_{\mathrm{R}} \gamma\left(\frac{4}{2 \gamma-1}\right)^{1 / 3} u(3 \gamma u-(1+\gamma))^{-2 / 3} .
\end{aligned}
$$

The final height $\psi_{e x i t}^{s}=\psi_{\mathrm{EF}}^{s}$ at which these small particles exit the breaking wave is given by $\phi=\phi_{\mathrm{E}}$ in (3.19).

Small particles starting below $\psi_{\mathrm{G}}$ will first enter the breaking wave across FG at a distance

$$
\xi_{\mathrm{FG}}^{s}=\xi_{\mathrm{F}}+\frac{\psi_{\mathrm{R}}-\psi_{\text {enter }}^{s}}{F^{\prime}(1)},
$$


and travel through the upper portion of the 'tail' FGAF where the concentration is given by characteristics (2.30). In a similar fashion to (3.17) above, these can be combined with $(3.3 b)$ to give the inhomogeneous differential equation

$$
\frac{\mathrm{d}}{\mathrm{d} \phi^{o}}\left(\left(F\left(\phi^{o}\right)-\phi^{o} F^{\prime}\left(\phi^{o}\right)\right) \xi^{s}\right)=-\phi^{o} \frac{\mathrm{d}}{\mathrm{d} \phi^{o}}\left(F^{\prime}\left(\phi^{o}\right) \xi_{\mathrm{FA}}(\phi)\right) .
$$

This is closely related to (3.18), and integrating by parts with the initial condition $\xi^{s}=\xi_{\mathrm{FG}}^{s}$ at $\phi=\phi_{\mathrm{E}}$ gives an almost identical form to $(3.19 a)$ for the $\xi$ coordinate

$$
\begin{aligned}
\xi^{s} & =\xi_{\mathrm{C}}+\frac{g_{1}\left(\phi_{\mathrm{E}}\right)\left(\xi_{\mathrm{FG}}^{s}-\xi_{\mathrm{C}}\right)+g_{2}(\phi)-g_{2}\left(\phi_{\mathrm{E}}\right)}{g_{1}(\phi)} \\
\psi^{s} & =\psi_{\mathrm{R}}-F^{\prime}\left(\phi^{o}\right)\left(\xi^{s}(\phi)-\xi_{\mathrm{FA}}(\phi)\right)
\end{aligned}
$$

Whether or not a small particle will travel through the 'lens' region is governed by a second critical initial height $\Psi_{\mathrm{A} *}^{s}$, at which a small particle passes through point A. Small particles starting above this critical height, $\Psi_{\mathrm{A} *}^{s}<\psi_{\text {enter }}^{s} \leqslant \psi_{\mathrm{G}}$ will pass through the upper portion of the 'tail' FGAF following (3.23), with $\phi \in\left[\phi_{\mathrm{E}}, \phi_{\mathrm{AG}}^{s}\right]$. Concentration $\phi_{\mathrm{AG}}^{s}$ and coordinates $\left(\xi_{\mathrm{AG}}^{s}, \psi_{\mathrm{AG}}^{s}\right)$ where the particle crosses AG can be found by equating (3.23) with a numerical form of (2.31) and (2.32). The equivalent concentration to $\phi_{\mathrm{AG}}^{s}$ on the right-hand side of shock $\mathrm{AG}$, at $\xi=\xi_{\mathrm{AG}}^{s}$ provides the initial condition for the motion through the upper part of the 'lens' FGBCA given by (2.19). The particles then pass across AC into the lower 'lens' and 'tail' regions and then exit across EF.

The small particles starting below the critical height $\Psi_{\mathrm{A} *}^{s}$ at $\psi_{\mathrm{R}}<\psi_{\text {enter }}^{s} \leqslant \Psi_{\mathrm{A} *}^{s}$ do not pass through the 'lens', but just recirculate within the 'tail'. Their motion through the upper portion of the 'tail' FGAF is given by (3.23) with $\phi \in\left[\phi_{\mathrm{E}}, \phi_{\mathrm{FA}}^{s}\right]$ until they cross FA at $\xi_{\mathrm{FA}}^{s}$. Concentration $\phi_{\mathrm{FA}}^{s}$ and position $\xi_{\mathrm{FA}}^{s}$ are both found by solving $\psi^{s}=\psi_{\mathrm{R}}$ in (3.23), and provide the initial condition for (3.18) in the lower portion of the 'tail'. The particle path replicates (3.19) with $\phi_{\mathrm{FA}}^{s}$ and $\xi_{\mathrm{FA}}^{s}$ replacing $\phi_{\mathrm{EA}}^{s}$ and $\xi_{\mathrm{EA}}^{s}$, and concentration $\phi$ in the range $\phi_{\mathrm{FA}}^{s} \leqslant \phi \leqslant \phi_{\mathrm{E}}$. As previously, the particle exits the breaking wave across $\mathrm{EF}$ at a final height $\psi_{\mathrm{EF}}^{s}$.

Figure 15 shows how the initial starting height of the large particles (superscript $l$ ) also determines which parts of the breaking wave they pass through. All the large particles start in the lower domain at a height $\psi_{\mathrm{R}}<\psi_{\text {enter }}^{l}<0$ (with $\hat{z}<\hat{z}_{\mathrm{R}}$ ) and travel upstream at this height before meeting $\mathrm{CD}$ at distance $\xi_{\text {enter }}^{l}=\xi_{\mathrm{CD}}^{l}$ given by (3.9). Upon entering the breaking wave, the large particles circulate through the lower portion of the 'lens' CDEAC following (3.10). Most of the coarse grains cross AC a distance $\xi_{\mathrm{AC}}^{l}$ given by (3.11), with the particle passing through point $\mathrm{A}$ (i.e. $\xi_{\mathrm{AC}}^{l}=\xi_{\mathrm{A}}$ ) defining a critical height $\Psi_{\mathrm{A} *}^{l}$

$$
\Psi_{\mathrm{A} *}^{l}=\psi_{\mathrm{R}}+\frac{1}{4}\left(\xi_{\mathrm{C}}-\xi_{\mathrm{A}}\right)
$$

Most of the large particles initially start above this critical height, $\psi_{\mathrm{R}}<\psi_{\text {enter }}^{l}<\Psi_{\mathrm{A} *}^{l}$, cross AC and follow paths in the upper portion of the 'lens' governed by (3.12). The initial condition that $\xi^{l}=\xi_{\mathrm{AC}}^{l}$ when $\phi=\phi_{\mathrm{R}}$ gives the path through the upper portion of the 'lens' AGBCA as 

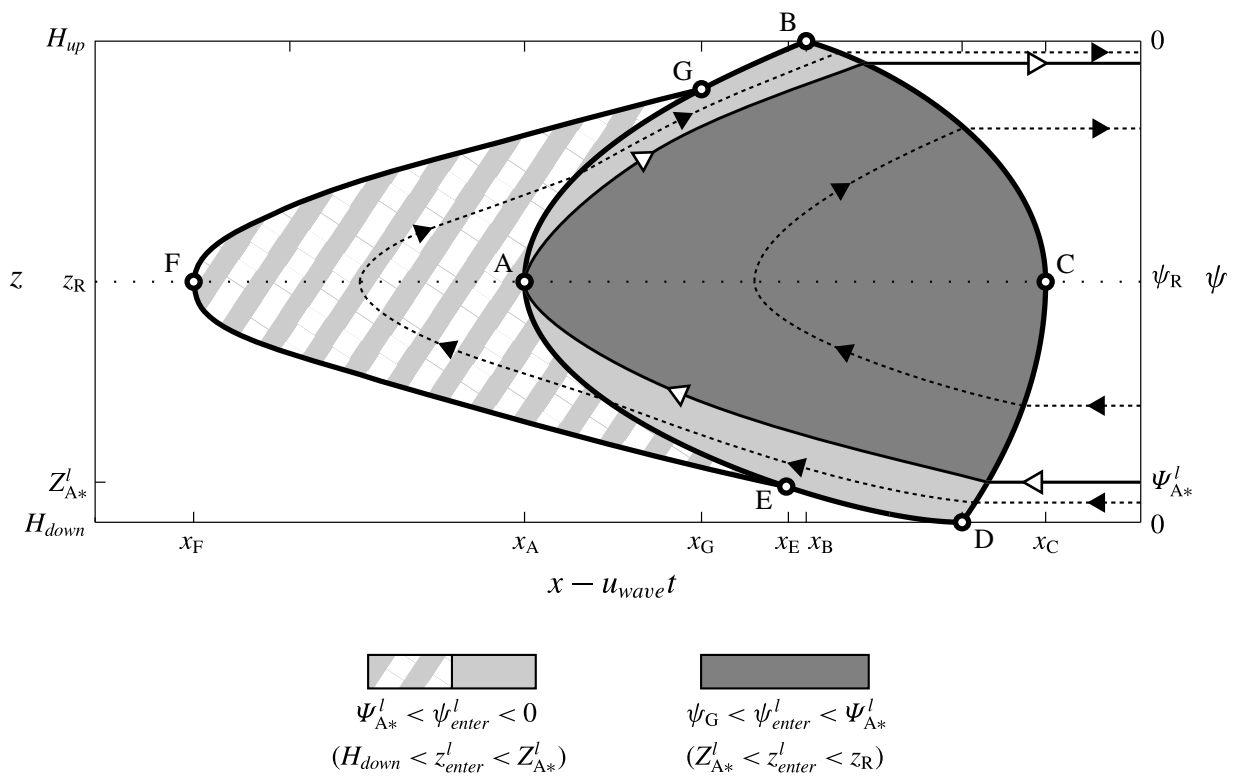

$$
\begin{aligned}
& \square \\
& \psi_{\mathrm{G}}<\psi_{\text {enter }}^{l}<\Psi_{\mathrm{A} *}^{l} \\
& \left(Z_{\mathrm{A} *}^{l}<Z_{\text {enter }}^{l}<Z_{\mathrm{R}}\right)
\end{aligned}
$$

FIGURE 15. A sketch showing how the large particles may pass through different parts of the 'lens-tail' structure depending on their initial starting height (not to scale). All of the large grains pass through the 'lens' region (shown with solid colour), but the large grains starting below the critical height $\Psi_{\mathrm{A} *}^{l}$ also pass through the 'tail' region (cross-shaded). Critical height $\Psi_{\mathrm{A} *}^{l}$ corresponds to the physical height $Z_{\mathrm{A} *}^{l}=z\left(\Psi_{\mathrm{A} *}^{l}\right)$. Two particle paths are shown with thin dashed lines and black arrows, whilst the particle path for the critical height $\Psi_{\mathrm{A} *}^{l}$, which passes through point $\mathrm{A}$, is shown with a thin solid line and white arrows. The structure of the breaking wave is shown with thick solid lines.

$$
\begin{aligned}
\xi^{l} & =\xi_{\mathrm{A}}+\frac{\psi_{\mathrm{R}}-\psi_{\text {enter }}^{l}+\frac{1}{4}\left(\xi_{\mathrm{C}}-\xi_{\mathrm{A}}\right)}{F(\phi)+(1-\phi) F^{\prime}(\phi)}, \\
\psi^{l} & =\psi_{\mathrm{R}}-F^{\prime}(\phi)\left(\xi^{l}-\xi_{\mathrm{A}}\right) .
\end{aligned}
$$

However, there are a few large particles that start below the critical height $\Psi_{\mathrm{A} *}^{l}<$ $\psi_{\text {enter }}^{l}<0$, and cross EA into the 'tail' region. Their initial motion through the 'lens' CDEAC is given by (3.10) with $\phi \in\left[0, \phi_{\mathrm{EA}}^{l}\right]$, where concentration $\phi_{\mathrm{EA}}^{l}$ and the point of crossing $\left(\xi_{\mathrm{EA}}^{l}, \psi_{\mathrm{EA}}^{l}\right)$ are found by equating (3.10) with (2.25). As with the small particles, the motion of the large particles through the lower part of the 'tail' EAFE is governed by characteristics (2.27) and local velocity (3.3a), which combine to give the governing differential equation

$$
\frac{\mathrm{d}}{\mathrm{d} \phi^{o}}\left(\left(F\left(\phi^{o}\right)+\left(1-\phi^{o}\right) F^{\prime}\left(\phi^{o}\right)\right) \xi^{l}\right)=\left(1-\phi^{o}\right) \frac{\mathrm{d}}{\mathrm{d} \phi^{o}}\left(\psi_{\mathrm{EA}}+F^{\prime}\left(\phi^{o}\right) \xi_{\mathrm{EA}}(\phi)\right) .
$$

This can be integrated by parts with $\xi^{l}=\xi_{\mathrm{EA}}^{l}$ at $\phi=\phi_{\mathrm{EA}}^{l}$ to give

$$
\begin{aligned}
\xi^{l} & =\xi_{\mathrm{C}}+\frac{g_{3}\left(\phi_{\mathrm{EA}}^{l}\right)\left(\xi_{\mathrm{EA}}^{l}-\xi_{\mathrm{C}}\right)+g_{4}(\phi)-g_{4}\left(\phi_{\mathrm{EA}}^{l}\right)}{g_{3}(\phi)}, \\
\psi^{l} & =\psi_{\mathrm{EA}}(\phi)-F^{\prime}\left(\phi^{o}\right)\left(\xi^{l}(\phi)-\xi_{\mathrm{EA}}(\phi)\right),
\end{aligned}
$$


where $\phi \in\left[\phi_{\mathrm{FA}}^{l}, \phi_{\mathrm{EA}}^{l}\right]$ and functions $g_{3}(u), g_{4}(u)$ are defined as

$$
\begin{aligned}
& g_{3}(u)=\frac{A_{\gamma}}{4 \gamma}(1-u)(1-\gamma-\gamma u)^{2}, \\
& g_{4}(u)=\psi_{\mathrm{R}} \gamma\left(\frac{4}{2 \gamma-1}\right)^{1 / 3}(1-u)(3 \gamma u-(1+\gamma))^{-2 / 3} .
\end{aligned}
$$

The coarse grains meet the no-mean-flow line FA at streamwise distance $\xi_{\mathrm{FA}}^{l}$ and concentration $\phi=\phi_{\mathrm{FA}}^{l}$, which are found from (3.27b) by solving $\psi^{l}=\psi_{\mathrm{R}}$. The motion through the upper 'lens' is governed by a similar differential equation to (3.26)

$$
\frac{\mathrm{d}}{\mathrm{d} \phi^{o}}\left(\left(F\left(\phi^{o}\right)+\left(1-\phi^{o}\right) F^{\prime}\left(\phi^{o}\right)\right) \xi^{l}\right)=\left(1-\phi^{o}\right) \frac{\mathrm{d}}{\mathrm{d} \phi^{o}}\left(F^{\prime}\left(\phi^{o}\right) \xi_{\mathrm{FA}}(\phi)\right)
$$

with $\phi=\phi_{\mathrm{FA}}^{l}$ at $\xi^{l}=\xi_{\mathrm{FA}}^{l}$ giving

$$
\begin{aligned}
& \xi^{l}=\xi_{\mathrm{C}}+\frac{g_{3}\left(\phi_{\mathrm{FA}}^{l}\right)\left(\xi_{\mathrm{FA}}^{l}-\xi_{\mathrm{C}}\right)+g_{4}(\phi)-g_{4}\left(\phi_{\mathrm{FA}}^{l}\right)}{g_{3}(\phi)}, \\
& \psi^{l}=\psi_{\mathrm{R}}-F^{\prime}\left(\phi^{o}\right)\left(\xi^{l}(\phi)-\xi_{\mathrm{FA}}(\phi)\right) .
\end{aligned}
$$

These grains then cross shock AG at point $\left(\xi_{\mathrm{AG}}^{l}, \psi_{\mathrm{AG}}^{l}\right)$, and move through the upper portion of the 'lens' AGBCA following

$$
\begin{aligned}
\xi^{l} & =\xi_{\mathrm{A}}+\frac{F\left(\phi_{\mathrm{AG}}^{l}\right)+\left(1-\phi_{\mathrm{AG}}^{l}\right) F^{\prime}\left(\phi_{\mathrm{AG}}^{l}\right)}{F(\phi)+(1-\phi) F^{\prime}(\phi)}\left(\xi_{\mathrm{AG}}^{l}-\xi_{\mathrm{A}}\right), \\
\psi^{l} & =\psi_{\mathrm{R}}-F^{\prime}(\phi)\left(\xi^{l}(\phi)-\xi_{\mathrm{A}}\right),
\end{aligned}
$$

where $\phi_{\mathrm{AG}}^{l}$ is the concentration on the right-hand side of shock AG at $\left(\xi_{\mathrm{AG}}^{l}, \psi_{\mathrm{AG}}^{l}\right)$. The particles finally cross $\mathrm{BC}$ at $\left(\xi_{\mathrm{BC}}^{l}, \psi_{\mathrm{BC}}^{l}\right)$ and exit the breaking wave at this height.

Like the recirculation in the 'lens', the small and large particle paths are parameterised by $\psi_{\text {enter }}^{s}$ and $\psi_{\text {enter }}^{l}$, respectively. The critical heights $\Psi_{\mathrm{E} *}^{s}, \Psi_{\mathrm{A} *}^{s}$ and $\Psi_{\mathrm{A} *}^{l}$ determine which of the 'lens' and 'tail' regions the particles pass through. A typical set of paths through the 'lens-tail' structure is shown in figure 13(c) for $\gamma=0.9$. Despite the 'tail' region, the paths of the small particles through the 'lens-tail' structure are qualitatively similar to the paths through the 'lens' shown in figures $13(a, b)$. However the additional motion of the large particles through the 'tail' region means that these paths are very different to previous cases. It is useful to analyse this further by calculating the recirculation times.

\subsection{Recirculation times}

Using the particle paths calculated in $\S \S 3.1$ and 3.2 , it is possible to numerically calculate the recirculation times for large and small particles to travel through the domain shown in figure 13. Small particles start at $x=-0.44$ in the upper domain $z>z_{\mathrm{R}}$ and travel downstream towards the breaking wave. They recirculate within the breaking wave, before travelling upstream and exiting across $x=-0.44$ in the lower domain $z<z_{\mathrm{R}}$. Despite the presence of the 'tail' region, there is no qualitative difference between the small particle recirculation times in any of the solutions. The large particles start at $x=0.1888$ in the lower domain and travel upstream. 


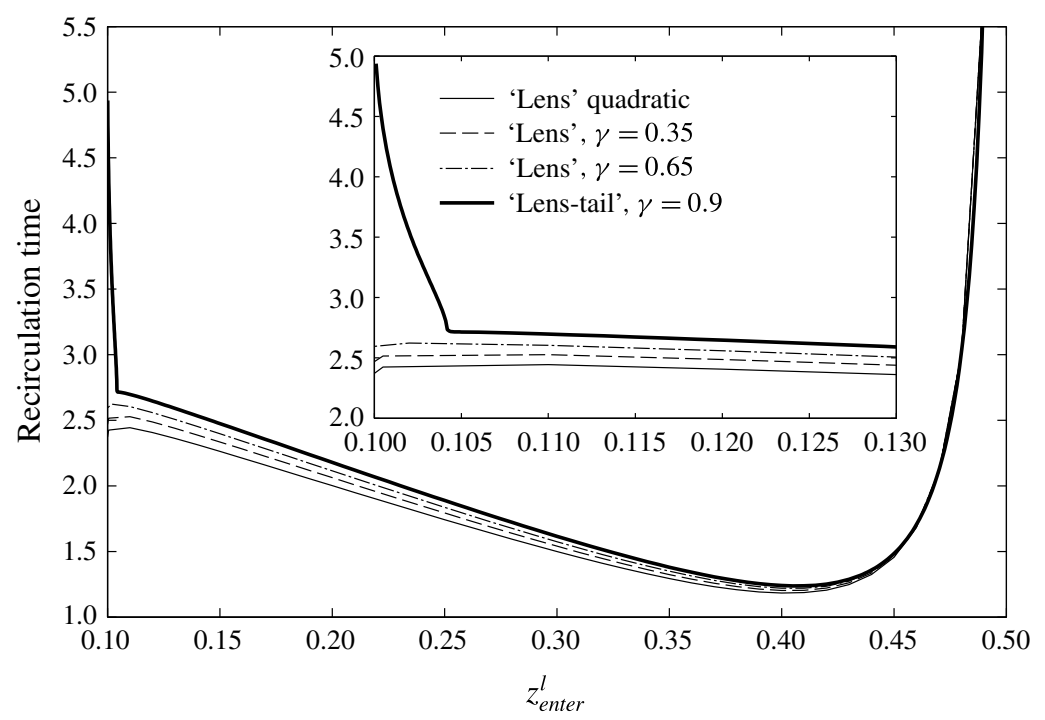

FIGURE 16. Recirculation time for large particles to travel through the region shown in figure 13. The particles start in the lower domain, downstream of the breaking wave at $x=0.1888$. In a frame translating with the breaking wave, the particles are swept upstream towards the wave, recirculate within the wave, before travelling downstream back to $x=0.1888$. The recirculation time increases significantly as $z_{\text {enter }}^{l}$ approaches 0.5 since the horizontal velocity $\hat{u}(z)$ tends to zero. The recirculation time also increases significantly for the 'lens-tail' structure as $z_{\text {enter }}^{l} \rightarrow H_{\text {down }}=0.1$ (inset), with large particles recirculating very slowly through the 'tail' region at the rear. This behaviour is unique to the 'lens tail' and it not found for any of the 'lens' structures.

They recirculate in the breaking wave, before travelling back downstream and exiting the region in the upper domain at $x=0.1888$. The recirculation times for these paths are shown in figure 16. The recirculation time tends to infinity as $z_{\text {enter }}^{l} \rightarrow 0.5$ for all the structures, since $\hat{u}(z)$ tends towards zero in this limit. The large particles spend an increasing amount of time travelling upstream towards the breaking wave and travelling downstream back towards $x=0.1888$ after recirculating. However, the 'lens-tail' structure also shows an increase in the recirculation time in the limit $z_{\text {enter }}^{l} \rightarrow H_{\text {down }}$, with large particles starting close to $H_{\text {down }}=0.1$ passing through the 'tail'. The concentration in the 'tail' is very close to $\phi=1$, hence $(1.3 a)$ implies that the vertical velocity is very low. Close to the no-mean-flow line $z=z_{\mathrm{R}}$, the horizontal velocity $\hat{u}(z)$ is very close to zero, so the large particles take a long time recirculating through the 'tail'. Although there is a smooth transition between the path length of a particle starting above $Z_{\mathrm{A} *}^{l}=z\left(\Psi_{\mathrm{A} *}^{l}\right)$ and travelling through just the 'lens', and a particle starting below $Z_{\mathrm{A} *}^{l}$ that passes through both the 'lens' and 'tail', there is a sharp change in the velocity of the latter particle as it crosses EA. This causes the large increase in the recirculation time at $z=0.1042$, with the large particle at this height passing through both the 'lens' and 'tail' regions.

\section{Conclusions}

Debris and pyroclastic flows typically exhibit large particle-rich fronts, which can be shouldered aside to form levees that laterally confine the flow and enhance its run out. The coarse grain fronts are able to exist and grow because of a complex recirculation 
zone just upstream of the front, which are known as a 'breaking size-segregation wave'. The recirculation zone is established because particle-size segregation enables overrun large particles to rise back up into the faster moving parts of the flow near the surface, whilst enabling the small particles that are sheared over the top of the large to percolate back down into the slower parts of the flow. The primary aim of this paper has been to examine exact solutions for the structure of the breaking wave using a continuum model with an asymmetric cubic segregation flux (1.5). The cubic flux is parameterised by a single parameter $\gamma$ which controls the difference between the maximum speed of a single small particle falling through a region of many large particles and the speed of a large particle rising through a region of many small grains (Gajjar \& Gray 2014; van der Vaart et al. 2015). For $0 \leqslant \gamma \leqslant 0.5$, the segregation flux is convex (up), whilst for $0.5<\gamma \leqslant 1$, it is non-convex, with the non-convexity allowing semi-shocks to form.

The method of characteristics is used to derive three fundamentally different solution structures shown in figures 10 and 12, which are dependent on the amount of asymmetry $\gamma$. Convex flux functions $(0 \leqslant \gamma \leqslant 0.5)$ give rise to a 'lens'-like solution formed of two shocks and two expansion fans. This is very similar to the 'lens' solution of Thornton \& Gray (2008); however, there is no rotational symmetry about the centre of the 'lens', and point B at the top of the breaking wave lies further downstream. Non-convex flux functions with a small amount of asymmetry $(0.5<\gamma \leqslant \Gamma=(5+\sqrt{5}) / 10)$ also give a 'lens' solution, with the only difference being that a semi-shock $A B$ separates the upper expansion fan from the upstream region of small particles, causing the top of the wave to lie even further downstream. Although the structure of the 'lens' is affected by the amount of asymmetry, the 'lens' has a constant length of $-4 \psi_{\mathrm{R}}$, irrespective of $\gamma$. The transformed no-mean-flow height $\psi_{\mathrm{R}}$ is only dependent on the velocity profile $u(z)$ and the vertical height of the wave $H_{u p}-H_{\text {down }}$, meaning that the length of the 'lens' solution is independent of the segregation flux. The length is thus the same in both the convex and non-convex cases, and is equal to the length of the breaking wave for the quadratic flux (Thornton $\&$ Gray 2008). The combination of higher amounts of asymmetry and the collective motion of large particles for $\Gamma<\gamma \leqslant 1$ produces a new 'lens-tail' structure. The 'tail' is formed by an additional expansion and compression wave upstream of the 'lens', and significantly increases the total length of the breaking wave. However the length of 'lens' region in the 'lens-tail' structure remains very close to the length of the 'lens' in the other solutions, i.e. $-4 \psi_{\mathrm{R}}$.

The precise asymmetric segregation flux must still be determined for the recirculating chute flow regime, but the results of this paper provide the general framework for deriving the breaking-wave structure. The three structures presented would be qualitatively the same for other (non-cubic) flux functions that satisfy the conditions of $\S 1.3$; namely that $F(\phi)$ has a maximum at $\phi_{\max }=\phi_{\mathrm{R}}$, with $0<\phi_{\max }<1 / 2$ and $F\left(\phi_{\max }\right)$, and that $F$ has at most one inflexion point in the interval $\left(\phi_{\max }, 1\right)$. The derivation of the structures is given in a general form, and may be extended to other flux functions. For example, the derivation of the convex 'lens' structure in $\S 2.1$ is more general than that of Thornton \& Gray (2008), and can be applied directly to other convex (non-cubic) flux functions, such as the asymmetric flux functions presented by Marks et al. (2012) and Tunuguntla et al. (2014). The non-convex 'lens' (\$2.2) would also apply directly to non-convex functions in which $\phi_{\mathrm{E}}(1.10 b)$ is greater than $\phi_{\mathrm{R}}$. Non-convex functions with $\phi_{\mathrm{E}}<\phi_{\mathrm{R}}$ would produce a 'lens-tail' solution that is qualitatively similar to figure $12(c)$. The analysis would also follow directly up to (2.24), with numerical integration needed for (2.24)-(2.36). 
The particle paths were calculated implicitly in terms of the local concentration $\phi$ and are shown in figure 13. The small and large particle paths are parameterised by the initial starting heights $\psi_{\text {enter }}^{s}$ and $\psi_{\text {enter }}^{l}$, with figures 14 and 15 illustrating the three critical heights $\Psi_{\mathrm{E} *}^{s}, \Psi_{\mathrm{A} *}^{s}$ and $\Psi_{\mathrm{A} *}^{l}$ for the 'lens-tail' structure that determine which of the 'lens' and 'tail' regions the particles pass through. Small particles starting above $Z_{\mathrm{E} *}^{s}=z\left(\Psi_{\mathrm{E} *}^{s}\right)$ and small particles starting below $Z_{\mathrm{A} *}^{s}=z\left(\Psi_{\mathrm{A} *}^{s}\right)$ pass through only the 'lens' and 'tail' regions, respectively, whilst small particles starting in $Z_{\mathrm{A} *}^{s} \leqslant z_{\text {enter }}^{s} \leqslant Z_{\mathrm{E} *}^{s}$ pass through both the 'lens' and 'tail' regions. Some small particles are able to recirculate only in the 'tail' region, but the large particles always travel through the 'lens' region. The majority of the large particles start above $Z_{\mathrm{A} *}^{l}=z\left(\Psi_{\mathrm{A} *}^{l}\right)$ and just travel through the 'lens', but a few large particles start below this height, and also pass through the 'tail' in addition to the lens. It is the motion of these large particles which has a significant effect on the recirculation time shown in figure 16. Although the recirculation time tends to infinity for large particles starting near the no-mean-flow line $z=z_{\mathrm{R}}$, the motion of large particles through the 'tail' in the 'lens-tail' structure also increases the recirculation time significantly for particles starting close to $z=H_{\text {down }}$, with the velocity in the 'tail' near the no-mean-flow line close to zero in both the normal and downstream components.

It was exactly this kind of behaviour that was observed in both the moving-bed flume experiments and numerical discrete particle method simulations in $\S 1.1$. Whilst most large particles recirculated quickly at the front, a few large particles recirculated much more slowly through regions of many small particles upstream, creating a 'tail'-like region. The correspondence between this behaviour and the new 'lens-tail' structure for the asymmetric cubic flux (1.5) in figure 6 shows that the flux function of van der Vaart et al. (2015) gives qualitative agreement in a very different physical environment, with their reported value of $\gamma=0.89$ producing a 'lens-tail' solution. As the asymmetry is essential for producing a 'tail', this paper, therefore, provides further physical evidence for the asymmetry between large and small particle segregation speeds, and shows how an asymmetric continuum model with a cubic flux (Gajjar \& Gray 2014; van der Vaart et al. 2015) captures all of the essential behaviour. The similarity between the physical experiments and numerical simulations shows that MercuryDPM could be a valuable tool in future research. Extensive experimental and numerical work is currently under way to analyse the particle motion within the breaking wave, which may also help to answer some wider segregation questions that remain unanswered in this framework. For instance, the robustness of the 'lens'-length $(1-\alpha)\left(H_{u p}-H_{\text {down }}\right)^{2} / S_{r}$ in all three structures suggests that the recirculating motion in a moving-bed flume could be a useful method of examining how the non-dimensional segregation coefficient $S_{r}$ varies with particle-size ratio. Although this relationship has been deduced from DPM simulations (Thornton et al. 2012b), an experimental verification is still lacking.

\section{Acknowledgements}

This research was supported by EPSRC Doctoral Training Grant EP/K502947/1, EPSRC research grants EP/I019189/1, EP/K00428X/1 and EP/M022447/1, NERC grant NE/K003011/1, and Royal Society grant WM150058, along with Swiss SNF grant no. 200021-149441. P.G. also acknowledges support from the Margaret Elizabeth Lee Fellowship. J.M.N.T.G. is a Royal Society Wolfson Research Merit Award holder and an EPSRC Established Career Fellow. 


\section{Appendix A. Features of hyperbolic solutions}

This appendix describes some of the features found in solutions to hyperbolic equations. For further general information on hyperbolic equations, the reader is referred to Rhee et al. (1986), whilst in particular Gajjar \& Gray (2014) provides further details of deriving exact solutions to segregation problems. Solutions may be constructed using the method of characteristics (Whitham 1974; Billingham \& King 2001), where the concentration $\phi$ is constant along characteristic curves, otherwise known as characteristics. Comparing the (quasi-linear) segregation (2.2),

$$
\hat{u} \frac{\partial \phi}{\partial \hat{x}}-\frac{\partial}{\partial \hat{z}} F(\phi)=0,
$$

with the full derivative of $\phi$ with respect to an arbitrary variable $s$

$$
\frac{\mathrm{d} \phi}{\mathrm{d} s}=\frac{\mathrm{d} \hat{x}}{\mathrm{~d} s} \frac{\partial \phi}{\partial \hat{x}}+\frac{\mathrm{d} \hat{z}}{\mathrm{~d} s} \frac{\partial \phi}{\partial \hat{z}},
$$

implies that the concentration $\phi$ is constant along characteristics curves given by

$$
\frac{\mathrm{d} \hat{x}}{\mathrm{~d} s}=\hat{u}, \quad \frac{\mathrm{d} \hat{z}}{\mathrm{~d} s}=-F^{\prime}(\phi) .
$$

These characteristics become straight lines under the transformation to depth-averaged velocity $(\xi, \psi)$ coordinates $(2.4)$

$$
\xi=\hat{x}, \quad \psi(\hat{z})=\int_{0}^{\hat{z}} \hat{u}\left(\hat{z}^{\prime}\right) \mathrm{d} \hat{z}^{\prime},
$$

with concentration $\phi$ equal to $\phi_{\lambda}$ along lines with constant gradient (2.6)

$$
\frac{\partial \psi}{\partial \xi}=-F^{\prime}\left(\phi_{\lambda}\right)=-A_{\gamma}\left(3 \gamma \phi_{\lambda}^{2}-2(1+\gamma) \phi_{\lambda}+1\right) .
$$

Note that $(\xi, \psi)$ coordinates are also streamfunction coordinates (Gray \& Ancey 2009). As shown in figure 17(a) characteristics of different concentrations may collide, leading to the formation of a shock. The shock has gradient (2.7)

$$
\frac{\mathrm{d} \psi}{\mathrm{d} \xi}=-\frac{\llbracket F(\phi) \rrbracket}{\llbracket \phi \rrbracket}
$$

with the 'jump' brackets $\llbracket f \rrbracket=f^{+}-f^{-}$mathematically representing the physical jump in concentration across the interface that is pictured in figure 17(b). Figure 17(c) shows how characteristics may diverge away from each other in a rarefaction fan. Physically, this leads to a smoothly varying concentration field, as shown in figure $17(d)$. Non-convex flux functions also give rise to a 'semi-shock' structure, in which characteristics lie parallel to the shock on one side, but collide with it on the other. One such 'semi-shock' is pictured in figure $17(e)$, with figure $17(f)$ showing how there is a smooth variation in the concentration between $\phi_{\mathrm{R}}$ and $\phi_{\mathrm{M}}$, before a sharp jump in the concentration from $\phi=\phi_{\mathrm{M}}$ to $\phi=1$. Finally, characteristics may also converge towards each other, without colliding, leading to the formation of a compression wave (figure $17 \mathrm{~g}$ ). This also leads to a smoothly varying concentration field, as shown in figure $17(h)$. These hyperbolic features are, however, idealised solutions to the full segregation equation. The stochastic, random-walk nature of particle motion in real world flows causes diffusive remixing between the two species that smooths over any sharp discontinuities in concentration that occur at shocks (Gray \& Chugunov 2006; Gray et al. 2015). 
(a)
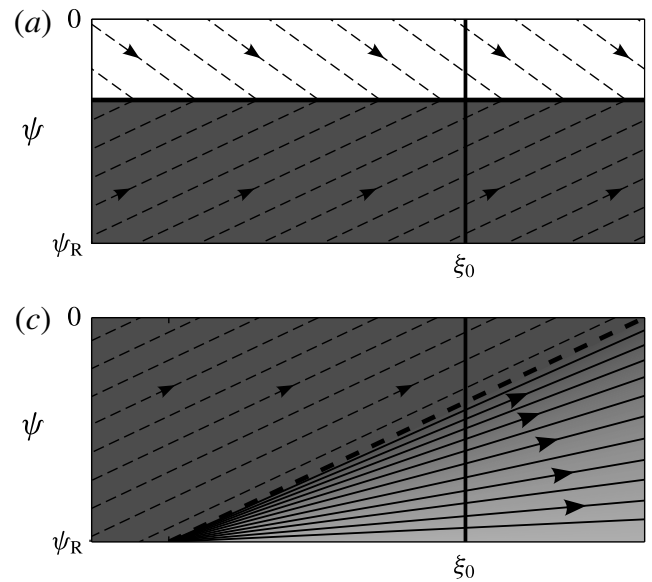

(e)

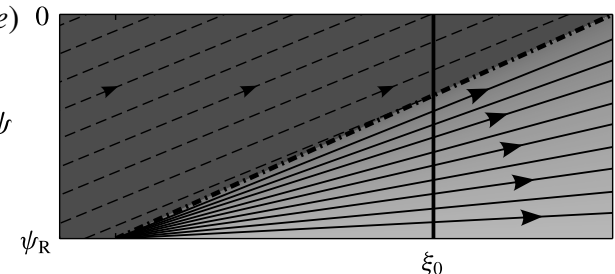

$(g)$

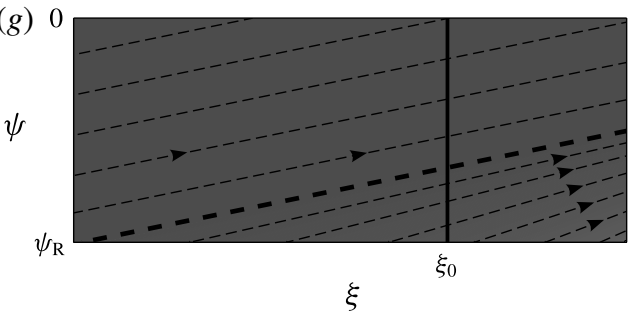

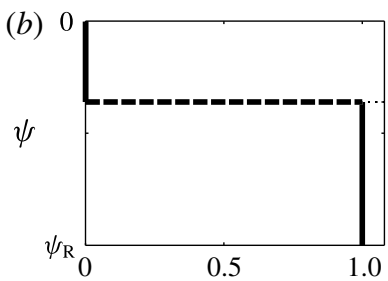
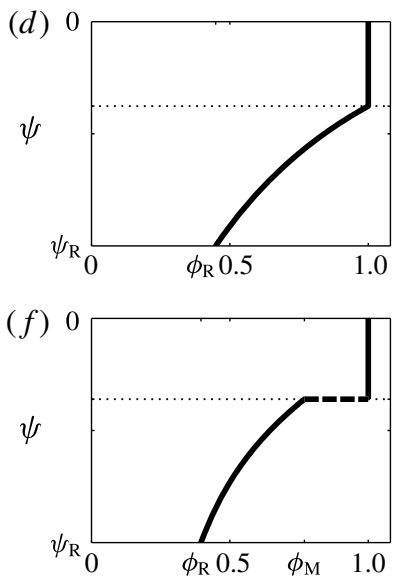

(h)

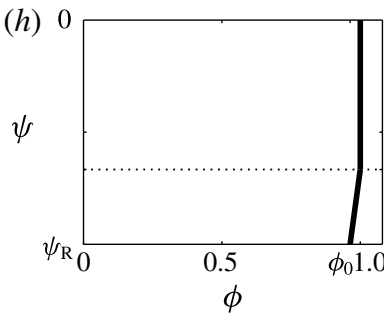

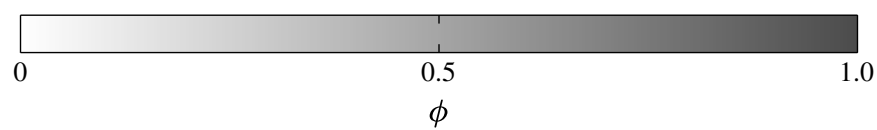

FIGURE 17. Exact solutions to hyperbolic equations may be constructed using the method of characteristics. Solutions are constructed by determining the position of characteristics, or lines upon which the concentration $\phi$ is constant, with figures $(a, c, e, g)$ showing the solutions in $(\xi, \psi)$ coordinates. These solutions may be physically visualised by plotting the concentration $\phi$ as a function of the height $\psi$ at a particular downstream position $\xi_{0}$, with sketches shown in figures $(b, d, f, h)$. Characteristics may collide and form a shock (a), which physically corresponds to a sharp jump in the concentration from $\phi=1$ below the shock to $\phi=0$ above the shock $(b)$. Characteristics may diverge in a rarefaction fan (c), giving a smoothly varying concentration field from $\phi=\phi_{\mathrm{R}}$ to $\phi=1(d)$. Non-convex segregation flux functions give rise to semi-shocks, with characteristics lying parallel to the semi-shock on the lower side, but colliding with it on the upper side $(e)$. There is a smoothly varying concentration field between $\phi=\phi_{\mathrm{R}}$ and $\phi=\phi_{\mathrm{M}}$, with a sharp jump in the concentration from $\phi=\phi_{\mathrm{M}}$ to $\phi=1$ at the height of the semi-shock $(f)$. Characteristics may also converge to form a compression wave $(g)$, which also leads to a smoothly varying concentration field $(h)$ from $\phi=\phi_{0}$ to $\phi=1$. 


\section{Appendix B. Derivation of equations governing shock AG}

Following Rhee et al. (1986), this appendix derives governing equations for the shock AG, as the concentration changes along both sides of the shock. The compression wave to the left of shock AG has characteristics of concentration $\phi_{\text {left }}$ given by (2.30)

$$
\psi=\psi_{\mathrm{R}}-F^{\prime}\left(\phi_{\text {left }}\right)\left(\xi-\tilde{\xi}_{\mathrm{FA}}\left(\phi_{\text {left }}\right)\right)
$$

where $\tilde{\xi}_{\mathrm{FA}}\left(\phi^{o}\right)=\xi_{\mathrm{FA}}(\phi)$. The rarefaction fan (2.17) to the right of shock AG has characteristics of concentration $\phi_{\text {right }}$,

$$
\psi=\psi_{\mathrm{R}}-F^{\prime}\left(\phi_{\text {right }}\right)\left(\xi-\xi_{\mathrm{F}}\right)
$$

whilst the shock condition (2.7) governs the gradient of $\mathrm{AG}$

$$
\frac{\mathrm{d} \psi}{\mathrm{d} \xi}=c\left(\phi_{\text {left }}, \phi_{\text {right }}\right)=-\frac{F\left(\phi_{\text {left }}\right)-F\left(\phi_{\text {right }}\right)}{\phi_{\text {left }}-\phi_{\text {right }}} .
$$

Treating $\phi_{\text {left }}$ and $\phi_{\text {right }}$ as independent variables and using the chain rule gives

$$
\frac{\mathrm{d} \psi}{\mathrm{d} \xi}=c\left(\phi_{\text {left }}, \phi_{\text {right }}\right)=\frac{\frac{\partial \psi}{\partial \phi_{\text {left }}}+\frac{\partial \psi}{\partial \phi_{\text {right }}} \frac{\mathrm{d} \phi_{\text {right }}}{\mathrm{d} \phi_{\text {left }}}}{\frac{\partial \xi}{\partial \phi_{\text {left }}}+\frac{\partial \xi}{\partial \phi_{\text {right }}} \frac{\mathrm{d} \phi_{\text {right }}}{\mathrm{d} \phi_{\text {left }}}},
$$

which, after rearranging, implies that

$$
\frac{\mathrm{d} \phi_{\text {right }}}{\mathrm{d} \phi_{\text {left }}}=-\frac{c\left(\phi_{\text {left }}, \phi_{\text {right }}\right) \frac{\partial \xi}{\partial \phi_{\text {left }}}-\frac{\partial \psi}{\partial \phi_{\text {left }}}}{c\left(\phi_{\text {left }}, \phi_{\text {right }}\right) \frac{\partial \xi}{\partial \phi_{\text {right }}}-\frac{\partial \psi}{\partial \phi_{\text {right }}}} .
$$

The compression wave and the rarefaction fan meet at the shock, and so (B 1) and (B 2) govern both the height $\psi_{\mathrm{AG}}$ and downstream position $\xi_{\mathrm{AG}}$ of the shock

$$
\begin{aligned}
\xi_{\mathrm{AG}}\left(\phi_{\text {left }}, \phi_{\text {right }}\right) & =\frac{F^{\prime}\left(\phi_{\text {left }}\right) \tilde{\xi}_{\mathrm{FA}}\left(\phi_{\text {left }}\right)-F^{\prime}\left(\phi_{\text {right }}\right) \xi_{\mathrm{F}}}{F^{\prime}\left(\phi_{\text {left }}\right)-F^{\prime}\left(\phi_{\text {right }}\right)}, \\
\psi_{\mathrm{AG}}\left(\phi_{\text {left }}, \phi_{\text {right }}\right) & =\psi_{\mathrm{R}}-\frac{F^{\prime}\left(\phi_{\text {left }}\right) F^{\prime}\left(\phi_{\text {right }}\right)}{F^{\prime}\left(\phi_{\text {left }}\right)-F^{\prime}\left(\phi_{\text {right }}\right)}\left(\tilde{\xi}_{\mathrm{FA}}\left(\phi_{\text {left }}\right)-\xi_{\mathrm{F}}\right) .
\end{aligned}
$$

Differentiating (B 6) gives

$$
\begin{aligned}
\frac{\partial \xi_{\mathrm{AG}}}{\partial \phi_{\text {left }}} & =-\frac{F^{\prime}\left(\phi_{\text {right }}\right) F^{\prime \prime}\left(\phi_{\text {left }}\right)}{\left(F^{\prime}\left(\phi_{\text {left }}\right)-F^{\prime}\left(\phi_{\text {right }}\right)\right)^{2}}\left(\tilde{\xi}_{\mathrm{FA}}\left(\phi_{\text {left }}\right)-\xi_{\mathrm{F}}\right)+\frac{F^{\prime}\left(\phi_{\text {left }}\right)}{F^{\prime}\left(\phi_{\text {left }}\right)-F^{\prime}\left(\phi_{\text {right }}\right)} \tilde{\xi}_{\mathrm{FA}}^{\prime}\left(\phi_{\text {left }}\right), \\
\frac{\partial \xi_{\mathrm{AG}}}{\partial \phi_{\text {right }}} & =\frac{F^{\prime}\left(\phi_{\text {left }}\right) F^{\prime \prime}\left(\phi_{\text {right }}\right)}{\left(F^{\prime}\left(\phi_{\text {left }}\right)-F^{\prime}\left(\phi_{\text {right }}\right)\right)^{2}}\left(\tilde{\xi}_{\mathrm{FA}}\left(\phi_{\text {left }}\right)-\xi_{\mathrm{F}}\right), \\
\frac{\partial \psi_{\mathrm{AG}}}{\partial \phi_{\text {left }}} & =\frac{\left(F^{\prime}\left(\phi_{\text {right }}\right)^{2}\right) F^{\prime \prime}\left(\phi_{\text {left }}\right)}{\left(F^{\prime}\left(\phi_{\text {left }}\right)-F^{\prime}\left(\phi_{\text {right }}\right)\right)^{2}}\left(\tilde{\xi}_{\mathrm{FA}}\left(\phi_{\text {left }}\right)-\xi_{\mathrm{F}}\right)-\frac{F^{\prime}\left(\phi_{\text {left }}\right) F^{\prime}\left(\phi_{\text {right }}\right)}{F^{\prime}\left(\phi_{\text {left }}\right)-F^{\prime}\left(\phi_{\text {right }}\right)} \tilde{\xi}_{\mathrm{FA}}^{\prime}\left(\phi_{\text {left }}\right),
\end{aligned}
$$


$\frac{\partial \psi_{\mathrm{AG}}}{\partial \phi_{\text {right }}}=-\frac{\left(F^{\prime}\left(\phi_{\text {left }}\right)\right)^{2} F^{\prime \prime}\left(\phi_{\text {right }}\right)}{\left(F^{\prime}\left(\phi_{\text {left }}\right)-F^{\prime}\left(\phi_{\text {right }}\right)\right)^{2}}\left(\tilde{\xi}_{\mathrm{FA}}\left(\phi_{\text {left }}\right)-\xi_{\mathrm{F}}\right)$,

which may be substituted into (B 5) to give (2.32)

$$
\begin{aligned}
\frac{\mathrm{d} \phi_{\text {right }}}{\mathrm{d} \phi_{\text {left }}}= & \frac{c\left(\phi_{\text {left }}, \phi_{\text {right }}\right)+F^{\prime}\left(\phi_{\text {right }}\right)}{c\left(\phi_{\text {left }}, \phi_{\text {right }}\right)+F^{\prime}\left(\phi_{\text {left }}\right)} \\
& \times\left\{\frac{F^{\prime}\left(\phi_{\text {right }}\right) F^{\prime \prime}\left(\phi_{\text {left }}\right)}{F^{\prime}\left(\phi_{\text {left }}\right) F^{\prime \prime}\left(\phi_{\text {right }}\right)}-\frac{F^{\prime}\left(\phi_{\text {left }}\right)-F^{\prime}\left(\phi_{\text {right }}\right)}{F^{\prime \prime}\left(\phi_{\text {right }}\right)} \frac{\tilde{\xi}_{\mathrm{FA}}^{\prime}\left(\phi_{\text {left }}\right)}{\tilde{\xi}_{\mathrm{FA}}\left(\phi_{\text {left }}\right)-\xi_{\mathrm{F}}}\right\} .
\end{aligned}
$$

As $\xi_{\mathrm{FA}}(\phi)=\tilde{\xi}_{\mathrm{FA}}\left(\phi^{o}\right),(2.28)$ and (1.9) for the cubic flux function give

$$
\begin{gathered}
\tilde{\xi}_{\mathrm{FA}}(\phi)=\xi_{\mathrm{C}}-\frac{\psi_{\mathrm{R}} \gamma}{F^{\prime}(\phi)\left((2 \gamma-1)(1+\gamma-3 \gamma \phi)^{2}\right)^{1 / 3}}, \\
\frac{\partial \tilde{\xi}_{\mathrm{FA}}(\phi)}{\partial \phi}=\psi_{\mathrm{R}} \gamma \frac{F^{\prime \prime}(\phi)(1+\gamma-3 \gamma \phi)-2 \gamma F^{\prime}(\phi)}{\left(F^{\prime}(\phi)\right)^{2}\left((2 \gamma-1)(1+\gamma-3 \gamma \phi)^{5}\right)^{1 / 3}} .
\end{gathered}
$$

Equations (B 12) and (B 13) may be used with (B 11) to numerically solve for the changing concentration along the shock path.

\section{REFERENCES}

Ancey, C., Coussot, P. \& Evesque, P. 1999 A theoretical framework for granular suspensions in a steady simple shear flow. J. Rheol. 43 (6), 1673-1699.

BAsco, D. 1985 A qualitative description of wave breaking. J. Waterways Port Coast. Ocean Div. ASCE 111 (2), 171-188.

Billingham, J. \& King, A. C. 2001 Wave Motion. Cambridge University Press.

BRIDgwater, J. 1976 Fundamental powder mixing mechanisms. Powder Technol. 15, 215-236.

BRIDGWATER, J. 1994 Mixing and segregation mechanisms in particle flow. In Granular Matter (ed. A. Mehta), pp. 161-193. Springer.

Bridgwater, J., Foo, W. \& Stephens, D. 1985 Particle mixing and segregation in failure zones theory and experiment. Powder Technol. 41, 147-158.

Cassar, C., Nicolas, M. \& Pouliquen, O. 2005 Submarine granular flows down inclined planes. Phys. Fluids 17 (10), 103301.

Clapham, C. \& Nicholson, J. 2009 The Concise Oxford Dictionary of Mathematics. Oxford University Press.

Costa, J. E. \& Williams, G. 1984 Debris flow dynamics. Tech. Rep. 84-606. (videotape) US Geological Survey.

Cundall, P. A. \& StRACK, O. D. L. 1979 A discrete numerical model for granular assemblies. Gèotechnique 29 (1), 47-65.

Dasgupta, P. \& Manna, P. 2011 Geometrical mechanism of inverse grading in grain-flow deposits: an experimental revelation. Earth-Sci. Rev. 104 (1-3), 186-198.

DAVIES, T. R. H. 1990 Debris-flow surges - experimental simulation. J. Hydrol. NZ. 29, 18-46.

Dijksman, J. A., Rietz, F., Lorincz, K. A., van Hecke, M. \& Losert, W. 2012 Invited article: refractive index matched scanning of dense granular materials. Rev. Sci. Instrum. $\mathbf{8 3}$ (1), 011301.

Dolgunin, V. N. \& Ukolov, A. A. 1995 Segregation modelling of particle rapid gravity flow. Powder Technol. 83, 95-103.

Forterre, Y. \& Pouliquen, O. 2008 Flows of dense granular media. Annu. Rev. Fluid Mech. 40 (1), 1-24. 
GajJar, P. \& Gray, J. M. N. T 2014 Asymmetric flux models for particle-size segregation in granular avalanches. J. Fluid Mech. 757, 297-329.

GDR MIDI 2004 On dense granular flows. Eur. Phys. J. E 14, 341-365.

GOLDHIRSCH, I. 2010 Stress, stress asymmetry and couple stress: from discrete particles to continuous fields. Granul. Matt. 12 (3), 239-252.

Golick, L. A. \& Daniels, K. E. 2009 Mixing and segregation rates in sheared granular materials. Phys. Rev. E 80 (4), 042301.

GRAY, J. M. N. T. \& ANCEY, C. 2009 Segregation, recirculation and deposition of coarse particles near two-dimensional avalanche fronts. J. Fluid Mech. 629, 387-423.

Gray, J. M. N. T. \& ANCEY, C. 2015 Particle-size and -density segregation in granular free-surface flows. J. Fluid Mech. 779, 622-668.

Gray, J. M. N. T. \& Chugunov, V. A. 2006 Particle-size segregation and diffusive remixing in shallow granular avalanches. J. Fluid Mech. 569, 365-398.

Gray, J. M. N. T, GajJar, P. \& KoKelaAR, P. 2015 Particle-size segregation in dense granular avalanches. C. R. Physique 16 (1), 73-85.

Gray, J. M. N. T. \& Hutter, K. 1997 Pattern formation in granular avalanches. Contin. Mech. Thermodyn. 9, 341-345.

Gray, J. M. N. T. \& KoKelaAR, B. P. $2010 a$ Erratum large particle segregation, transport and accumulation in granular free-surface flows - erratum. J. Fluid Mech. 657, 539.

Gray, J. M. N. T. \& KoKelaAR, B. P. $2010 b$ Large particle segregation, transport and accumulation in granular free-surface flows. J. Fluid Mech. 652, 105-137.

Gray, J. M. N. T., Shearer, M. \& Thornton, A. R. 2006 Time-dependent solutions for particlesize segregation in shallow granular avalanches. Proc. R. Soc. Lond. A 462, 947-972.

Gray, J. M. N. T., TAI, Y. C. \& Noelle, S. 2003 Shock waves, dead-zones and particle-free regions in rapid granular free-surface flows. J. Fluid Mech. 491, 161-181.

Gray, J. M. N. T. \& Thornton, A. R. 2005 A theory for particle size segregation in shallow granular free-surface flows. Proc. R. Soc. Lond. A 461, 1447-1473.

IVERSON, R. M. 1997 The physics of debris-flows. Rev. Geophys. 35, 245-296.

IVERSON, R. M. 2005 Regulation of landslide motion by dilatancy and pore pressure feedback. J. Geophys Res. Earth 110 (F2), F02015.

IVerson, R. M. 2014 Debris flows: behaviour and hazard assessment. Geol. Today 30 (1), 15-20.

IVERSON, R. M. \& LAHUSEN, R. G. 1989 Dynamic pore-pressure fluctuations in rapidly shearing granular materials. Science 246 (4931), 796-799.

Iverson, R. M., Logan, M., LaHusen, R. G. \& Berti, M. 2010 The perfect debris flow? Aggregated results from 28 large-scale experiments. J. Geophys. Res. 115, F03005.

IVerson, R. M. \& Vallance, J. W. 2001 New views of granular mass flows. Geology 29 (2), $115-118$.

Johnson, A. M. 1970 Physical Processes in Geology. Freeman, Cooper \& Company.

Johnson, A. M. 1984 Debris flows. In Slope Instability. Wiley.

Johnson, C. G., Kokelaar, B. P., Iverson, R. M., Logan, M., LaHusen, R. G. \& Gray, J. M. N. T. 2012 Grain-size segregation and levee formation in geophysical mass flows. J. Geophys. Res. 117, F01032.

Kokelaar, B. P., Graham, R. L., Gray, J. M. N. T. \& Vallance, J. W. 2014 Fine-grained linings of leveed channels facilitate runout of granular flows. Earth Planet. Sci. Lett. 385, $172-180$.

LeVeque, R. J. 2002 Finite Volume Methods for Hyperbolic Problems. Cambridge University Press.

MAJor, J. J. \& IVERson, R. M. 1999 Debris-flow deposition: effects of pore-fluid pressure and friction concentrated at flow margins. Geol. Soc. Am. Bull. 111 (10), 1424-1434.

Marks, B., Rognon, P. \& EInAV, I. 2012 Grainsize dynamics of polydisperse granular segregation down inclined planes. J. Fluid Mech. 690, 499-511.

MAY, L. B. H., SheARer, M. \& DANiels, K. E. 2010 Scalar conservation laws with nonconstant coefficients with application to particle size segregation in granular flow. J. Nonlinear Sci. 20 (6), 689-707. 
McDonald, S., Harris, D. \& Withers, P. 2012 In situ x-ray microtomography study of the movement of a granular material within a die. Intl J. Mater. Res. 103 (2), 162-169.

Mcintyre, M., Rowe, E., Shearer, M., Gray, J. M. N. T. \& Thornton, A. R. 2007 Evolution of a mixing zone in granular avalanches. Appl. Math. Res. Express 2007, abm008.

Morland, L. W. 1992 Flow of viscous fluids through a porous deformable matrix. Surv. Geophys. 13 (3), 209-268.

Muite, B. K., Hunt, M. L. \& Joseph, G. G. 2004 The effects of a counter-current interstitial flow on a discharging hourglass. Phys. Fluids 16 (9), 3415-3425.

OLEINIK, O. A. 1959 Uniqueness and stability of the generalized solution of the cauchy problem for a quasi-linear equation. Usp. Mat. Nauk 14, 165-170.

Pailha, M., Nicolas, M. \& Pouliquen, O. 2008 Initiation of underwater granular avalanches: influence of the initial volume fraction. Phys. Fluids 20 (11), 111701.

Pailha, M. \& Pouliquen, O. 2009 A two-phase flow description of the initiation of underwater granular avalanches. J. Fluid Mech. 633, 115-135.

Pierson, T. C. 1986 Flow behavior of channelized debris flows, Mount St. Helens, Washington. In Hillslope Processes (ed. A. D. Abrahams), pp. 269-296. Allen and Unwin.

du Pont, S. Courrech, Gondret, P., Perrin, B. \& Rabaud, M. 2003 Granular avalanches in fluids. Phys. Rev. Lett. 90, 044301.

Pouliquen, O. $1999 a$ On the shape of granular fronts down rough inclined planes. Phys. Fluids 11 (7), 1956-1958.

Pouliquen, O. 1999b Scaling laws in granular flows down rough inclined planes. Phys. Fluids 11 (3), 542-548.

Pouliquen, O., Delour, J. \& Savage, S. B. 1997 Fingering in granular flows. Nature 386, $816-817$.

Pouliquen, O.\& Vallance, J. W. 1999 Segregation induced instabilities of granular fronts. Chaos 9 (3), 621-630.

Rhee, H. K., Aris, R. \& Amundson, N. R. 1986 First-order Partial Differential Equations: Volume 1 Theory and Applications of Single Equations. Prentice-Hall.

Rognon, P. G., Roux, J. N., NAaim, M. \& Chevoir, F. 2007 Dense flows of bidisperse assemblies of disks down an inclined plane. Phys. Fluids 19 (5), 058101.

SAVAGE, S. B. \& HutTER, K. 1989 The motion of a finite mass of granular material down a rough incline. J. Fluid Mech. 199, 177-215.

Savage, S. B. \& Lun, C. K. K. 1988 Particle size segregation in inclined chute flow of dry cohesionless granular solids. J. Fluid Mech. 189, 311-335.

SHAND, T. D. 2009 The effect of wave grouping on shoaling and breaking processes. PhD thesis, Civil \& Environmental Engineering, Faculty of Engineering, University of New South Wales.

Sharp, R. P. \& Nobles, L. H. 1953 Mudflow of 1941 at Wrightwood, Southern California. Geol. Soc. Am. Bull. 64 (5), 547-560.

Staron, L. \& Phillips, J. C. 2014 Segregation time-scale in bi-disperse granular flows. Phys. Fluids 26 (3), 033302.

TAKAhashi, T. 1980 Debris flow on prismatic open channel. J Hydraul. Div. ASCE 106 (3), 381-396.

Thornton, A. R. \& GRAY, J. M. N. T. 2008 Breaking size-segregation waves and particle recirculation in granular avalanches. J. Fluid Mech. 596, 261-284.

Thornton, A. R., Gray, J. M. N. T. \& HogG, A. J. 2006 A three-phase mixture theory for particle size segregation in shallow granular free-surface flows. J. Fluid Mech. 550, 1-25.

Thornton, A. R., Krijgsman, D., Fransen, R., Gonzalez, S., Tunuguntla, D., ten Voortwis, A., Luding, S., Bokhove, O. \& Weinhart, T. 2013a Mercury-DPM: fast particle simulations in complex geometries. Newsl. EnginSoft Year 10 1, 48-53.

Thornton, A. R., Krijgsman, D., te Voortwis, A., Ogarko, V., Luding, S., Fransen, R., Gonzalez, S. I., Bokhove, O., Imole, O. \& Weinhart, T. $2013 b$ A review of recent work on the Discrete Particle Method at the University of Twente: an introduction to the open- source package MercuryDPM. In Proceedings of DEM6 - 6th International Conference on Discrete Particle Method. 
Thornton, A. R., Weinhart, T., Luding., S. \& Bokhove, O. $2012 a$ Frictional dependence of shallow-granular flows from discrete particle simulations. Eur. Phys. J. E 35 (12), 127.

Thornton, A. R., Weinhart, T., Luding, S. \& Bokhove, O. $2012 b$ Modeling of particle size segregation: calibration using the discrete particle method. Intl J. Mod. Phys. C 23 (8), 1240014.

Tóth, G. \& OdstrČIL, D. 1996 Comparison of some flux corrected transport and total variation diminishing numerical schemes for hydrodynamic and magnetohydrodynamic problems. J. Comput. Phys. 128 (1), 82-100.

Tunuguntla, D., Bokhove, O. \& Thornton, A. R. 2014 A mixture theory for size and density segregation in shallow granular free-surface flows. J. Fluid Mech. 749, 99-112.

Tunuguntla, D., Thornton, A. R. \& Weinhart, T. 2015 From discrete elements to continuum fields: extension to bidisperse systems. Comput. Part. Mech. 1-17; doi:10.1007/s40571-015-0087-y.

Turnbull, B., Bowman, E. T. \& McElwaine, J. N. 2015 Debris flows: experiments and modelling. C. R. Physique 16 (1), 86-96.

Van der VaArt, K., Gajuar, P., Epely-Chauvin, G., Andreini, N., Gray, J. M. N. T. \& ANCEY, C. 2015 Underlying asymmetry within particle size segregation. Phys. Rev. Lett. 114, 238001 .

Vallance, J. W. \& Savage, S. B. 2000 Particle segregation in granular flows down chutes. In IUTAM Symposium on Segregation in Granular Materials (ed. A. D. Rosato \& D. L. Blackmore). Kluwer.

Weinhart, T., HartKamp, R., Thornton, A. R. \& Luding, S. 2013 Coarse-grained local and objective continuum description of three-dimensional granular flows down an inclined surface. Phys. Fluids 25 (7), 070605.

Weinhart, T., Thornton, A. R., Luding, S. \& Bokhove, O. 2012 Closure relations for shallow granular flows from particle simulations. Granul. Matt. 14 (4), 531-552.

Whitham, G. B. 1974 Linear and Nonlinear Waves. John Wiley.

Wiederseiner, S., ANdreini, N., Epely-Chauvin, G. \& AnCEy, C. $2011 a$ Refractive-index and density matching in concentrated particle suspensions: a review. Exp. Fluids 50 (5), 1183-1206.

Wiederseiner, S., Andreini, N., Epely-Chauvin, G., Moser, G., Monnereau, M., Gray, J. M. N. T. \& ANCEY, C. $2011 b$ Experimental investigation into segregating granular flows down chutes. Phys. Fluids 23, 013301.

Wieland, M., Gray, J. M. N. T. \& Hutter, K. 1999 Channelised free surface flow of cohesionless granular avalanches in a chute with shallow lateral curvature. J. Fluid Mech. 392, 73-100.

Woodhouse, M. J., Thornton, A. R., Johnson, C. G., KokelaAr, B. P. \& Gray, J. M. N. T. 2012 Segregation-induced fingering instabilities in granular free-surface flows. J. Fluid Mech. 709, 543-580.

YeE, H. C. 1989 A class of high resolution explicit and implicit shock-capturing methods. Tech Rep. NASA-TM-101088. NASA Ames Research Center, NASA Ames Research Center; Moffett Field, CA, United States, doc ID: 19890016281. 